(C) 2018. This manuscript version is made available under the CC-BY-NC-ND 4.0 license http://creativecommons.org/licenses/by-nc-nd/4.0

\title{
Formalization and Correctness of Predictive Shift-Reduce Parsers for Graph Grammars based on Hyperedge Replacement ${ }^{\text {咨 }}$
}

\author{
Frank Drewes ${ }^{\mathrm{a}}$, Berthold Hoffmann ${ }^{\mathrm{b}}$, Mark Minas ${ }^{\mathrm{c}}$ \\ ${ }^{a}$ Institutionen för datavetenskap, Umeå universitet, SE-901 87 Umeå, Sweden \\ ${ }^{b}$ Fachbereich 3-Informatik, Universität Bremen, D-28334 Bremen, Germany \\ ${ }^{c}$ Institut für Softwaretechnologie, Fakultät für Informatik, \\ Universität der Bundeswehr München D-85577 Neubiberg, Germany
}

\begin{abstract}
Hyperedge replacement (HR) grammars can generate NP-complete graph languages, which makes parsing hard even for fixed HR languages. Therefore, we study predictive shift-reduce (PSR) parsing that yields efficient parsers for a subclass of HR grammars, by generalizing the concepts of SLR(1) string parsing to graphs. We formalize the construction of PSR parsers and show that it is correct. PSR parsers run in linear space and time, and are more efficient than the predictive top-down (PTD) parsers recently developed by the authors.
\end{abstract}

Keywords: hyperedge replacement grammar, graph parsing, grammar analysis

\section{Introduction}

Everywhere in science and beyond, diagrams occur as a means of illustration and explanation. In computer science and engineering, they are also used as primary source of information: they form visual specification languages with a precise syntax and semantics. For instance, the diagrams of the Uniform Modeling Language UML specify software artifacts. (See www.uml.org.) When diagram languages shall be processed by computers, techniques of compiler construction have to be transferred to the domain of diagrams. A processor of a textual language parses its syntax, which is specified by a context-free Chomsky grammar, in order to construct an abstract hierarchical representation that can then be further interpreted or translated. The syntax of a diagram language is its structure. To analyze the structure of diagrams, one thus needs grammars to specify their syntax, and parsers for these grammars that perform the analysis. A successfully parsed diagram can eventually be processed further. Since

\footnotetext{
This paper formalizes the concepts described in [14] and provides detailed correctness proofs for them.

Email addresses: drewes@cs.umu.se (Frank Drewes), hof@uni-bremen.de (Berthold Hoffmann), mark.minas@unibw.de (Mark Minas)
}

Preprint submitted to Journal of Logical and Algebraic Methods in Programming Dec. 21, 2018 
diagrams can be represented as graphs, their syntax can be captured by graph grammars.

Here we consider hyperedge replacement (HR) graph grammars. ${ }^{1}$ Hyperedges are a generalization of edges that may connect any number of nodes, not just two. In a host graph $g$, the replacement of a hyperedge $\boldsymbol{e}$ by a graph $\gamma$ glues the nodes connected to $\boldsymbol{e}$ to distinguished nodes of $\gamma$. The context-free case, where the replacement depends just on the label of $\boldsymbol{e}$ (a nonterminal symbol) is well studied [22]. Unfortunately, hyperedge replacement can generate NP-complete graph languages [1]. In other words, even for fixed HR languages parsing is hard. Moreover, even if restrictions are employed that guarantee an HR language $L$ to be in $\mathrm{P}$, the degree of the polynomial depends on $L$; see [27]. ${ }^{2}$ Only under rather strong restrictions the problem is known to be solvable in cubic time $[34,9]$.

Since even a cubic algorithm would not scale to diagrams occurring in realistic applications, the authors have recently transferred results of context-free string parsing to graphs: Simple LL-parsing (SLL $(k)$ for short, [28]), a top-down parsing method that applies to a subclass of unambiguous context-free string grammars (using $k$ symbols of lookahead), has been lifted to predictive top-down parsing of graphs (PTD parsing for short, [12]); the program generating PTD parsers approximates Parikh images of auxiliary grammars in order to determine whether a grammar is PTD-parsable [13], and generates parsers that run in quadratic time, and in many cases in linear time.

In this paper, we devise - somewhat complementary - efficient bottom-up parsers for HR grammars, called predictive shift-reduce (PSR) parsers, which extend SLR(1) parsers [8], a member of the LR $(k)$ family of deterministic bottomup parsers for context-free string grammars [26]. We formalize the construction and modus operandi of PSR parsers and show their correctness.

In Sect. 2 we recall basic notions of HR grammars. To support intuition, we briefly recall SLR(1) string parsing in Sect. 3. In Sections 4-9, we work out in detail how it can be lifted to PSR parsing:

Section 4 develops a naïve shift-reduce parser for HR grammars and shows its correctness. This parser is a stack automaton that, one by one, reads the edges of the input graph and simply "guesses" nondeterministically a backwards application of rules that takes the input graph to the start symbol. While this parser is correct, its nondeterminism renders it impractical. One of its disadvantages is that it can run into "dead ends", situations which can never lead to acceptance, regardless of the remaining input.

Section 5 defines a notion of viable prefixes and shows that the naïve shiftreduce parser would avoid running into a dead end if and only if one could make sure that its stack does always contain a viable prefix.

\footnotetext{
${ }^{1}$ Other graph grammars and parsing algorithms are discussed in Sect. 10.

${ }^{2}$ The polynomial algorithm for a restricted class of (fixed) HR grammars presented in [27] was refined in [4] and implemented in the system Bolinas for semantic parsing in natural language processing.
} 
Section 6 thus develops a notion of nondeterministic characteristic finite automaton (nCFA) and shows that it recognizes (we say approves) exactly the viable prefixes. However, since the nCFA is itself nondeterministic, it cannot reasonably be used in order to improve the naïve shift-reduce parser.

Section 7, therefore, shows how the nCFA can be converted into a deterministic characteristic finite automaton (dCFA) that is equivalent to the nCFA.

Section 8 incorporates the dCFA into an improved version of the naïve shiftreduce parser. The $d C F A$-assisted shift-reduce parser makes sure that it can always continue a parse found so far to a successful parse, but possibly not with the current input graph, i.e., it may still run into a dead end.

Finally, Sect. 9 discusses how dCFA-assisted shift-reduce parsers can be further extended to predictive shift-reduce parsers (PSR parsers) which can predict their next move in every situation such that they can never run into dead ends. These parsers, for all practical purposes, run in linear time and space, but they exist only for HR grammars without conflicts and satisfying the so-called free edge choice property. Sect. 9 formalizes conflicts as well the free edge choice property, and shows how conflicts can be detected.

Related and future work is discussed in Sect. 10.

This paper formalizes the concepts developed in [14] and provides detailed correctness proofs for them. We would also like to mention that the proof of Theorem 1 of [14] turned out to be wrong.

Acknowledgment. We thank the reviewers for their comments and criticism; we hope to have made good use of them.

\section{Hyperedge Replacement Grammars}

We let $\mathbb{N}$ denote the non-negative integers. For set $A$ and $B$, let $2^{A}$ denote the powerset of $A$ and $(A \rightarrow B)$ the set of all partial functions from $A$ to $B$. The domain of a partial function $f: A \rightarrow B$ is denoted by $\operatorname{dom}(f)$, i.e., $\operatorname{dom}(f)=$ $\{a \in A \mid f(a)$ is defined $\}$. For $S \subseteq A$, we let $f(S)=\{f(a) \mid a \in S \cap \operatorname{dom}(f)\}$. Given two partial functions $f$ and $g$, we write $f \sqsubseteq g$ if $f \subseteq g$ as binary relations. The composition $g \circ f$ of (possibly partial) functions $f: A \rightarrow B$ and $g: B \rightarrow C$ is defined as usual, i.e., $(g \circ f)(a)$ equals $g(f(a))$ if both $f(a)$ and $g(f(a))$ are defined, and is undefined otherwise.

$A^{*}$ denotes the set of all finite sequences (or strings) over a set $A$; the empty sequence is denoted by $\varepsilon$ and the length of a sequence $\alpha$ by $|\alpha|$.

For a (total) function $f: A \rightarrow B$, its extension $f^{*}: A^{*} \rightarrow B^{*}$ to sequences is defined by $f^{*}\left(a_{1} \cdots a_{n}\right)=f\left(a_{1}\right) \cdots f\left(a_{n}\right)$, for all $a_{1}, \ldots, a_{n} \in A, n \geq 0$. Given a relation $\rightsquigarrow \subseteq A \times A$, we denote its $n$-fold composition with itself by $\rightsquigarrow^{n}$ (where $\rightsquigarrow^{0}$ is the identity on $A$ ), its transitive closure by $\rightsquigarrow^{+}$and its reflexive and transitive closure by $\rightsquigarrow^{*}$, as usual.

Throughout the paper, we let $X$ denote a global, countably infinite supply of nodes or vertices. 
Definition 2.1 (Graph). An alphabet is a set $\Sigma$ of symbols together with an arity function arity: $\Sigma \rightarrow \mathbb{N}$. Given such an alphabet, a literal $\boldsymbol{e}=a\left(x_{1}, \ldots, x_{k}\right)$ over $\Sigma$ consists of a symbol $a \in \Sigma$ and $k=\operatorname{arity}(a)$ pairwise distinct nodes $x_{1}, \ldots, x_{k} \in X$. We write $\ell(\boldsymbol{e})=a$ and denote the set of all literals over $\Sigma$ by Lit $_{\Sigma}$.

A graph $\gamma=\langle V, \varphi\rangle$ over $\Sigma$ consists of a finite set $V \subseteq X$ of nodes and a sequence $\varphi=e_{1} \cdots e_{n} \in L i t_{\Sigma}^{*}$ such that all nodes in these literals are in $V$. $\mathcal{G}_{\Sigma}$ denotes the set of all graphs over $\Sigma$.

We say that two graphs $\gamma=\langle V, \varphi\rangle$ and $\gamma^{\prime}=\left\langle V^{\prime}, \varphi^{\prime}\right\rangle$ are equivalent, written $\gamma \bowtie \gamma^{\prime}$, if $V=V^{\prime}$ and $\varphi$ is a permutation of $\varphi^{\prime}$.

Note that graphs are sequences rather than sets of literals, i.e., two graphs $\langle V, \varphi\rangle$ and $\left\langle V^{\prime}, \varphi^{\prime}\right\rangle$ with the same set of nodes, but with different sequences of literals are considered to differ, even if $V=V^{\prime}$ and $\varphi^{\prime}$ is just a permutation of $\varphi$. However, such graphs are equivalent, denoted by the equivalence relation $\bowtie$. In contrast, "ordinary" graphs would rather be represented using multisets of literals instead of sequences. The equivalence classes of graphs, therefore, correspond to conventional graphs. The ordering of literals is technically convenient for the constructions in this paper. However, input graphs to be parsed should of course be considered up to equivalence. Thus, we will make sure that the developed parsers yield identical results on graphs $g, g^{\prime}$ with $g \bowtie g^{\prime}$.

For a graph $\gamma=\langle V, \varphi\rangle$, we use the notations $X(\gamma)=V$ and $\operatorname{lit}(\gamma)=\varphi$. An injective function $\varrho: X \rightarrow X$ is called a renaming, and $\gamma^{\varrho}$ denotes the graph obtained by replacing all nodes in $\gamma$ according to $\varrho$. Although renamings are, for technical simplicity, defined as functions on the whole of $X$, in every concrete situation only a finite subset of $X$ will be relevant. The same holds when we, later on in the paper, consider partial functions $\mu: X \rightarrow X$.

We define the "concatenation" of two graphs $\alpha, \beta \in \mathcal{G}_{\Sigma}$ as $\alpha \beta=\langle X(\alpha) \cup$ $X(\beta), \operatorname{lit}(\alpha) \operatorname{lit}(\beta)\rangle$. A graph $\gamma$ is a prefix of graph $\alpha$ if there is a graph $\delta$ such that $\alpha=\gamma \delta$. Thus, a prefix is a particular kind of subgraph. If a graph $\gamma$ is completely determined by its sequence $\operatorname{lit}(\gamma)$ of literals, i.e., if each node in $X(\gamma)$ also occurs in some literal in lit $(\gamma)$, we simply use $\operatorname{lit}(\gamma)$ as a shorthand for $\gamma$. In particular, a literal $\boldsymbol{e} \in$ Lit $_{\Sigma}$ is identified with the graph consisting of just this literal and its nodes.

Definition 2.2 (HR Grammar). Let $\Sigma=\mathcal{N} \uplus \mathcal{T}$ be an alphabet which is partitioned into disjoint subsets $\mathcal{N}$ and $\mathcal{T}$ of nonterminals and terminals, respectively. A hyperedge replacement rule $r=(\boldsymbol{A} \rightarrow \alpha)$ (a rule for short) has a literal $\boldsymbol{A} \in$ Lit $_{\mathcal{N}}$ as its left-hand side, and a graph $\alpha \in \mathcal{G}_{\Sigma}$ with $X(\boldsymbol{A}) \subseteq X(\alpha)$ as its right-hand side.

Consider a graph $\gamma=\beta \boldsymbol{A}^{\prime} \beta^{\prime} \in \mathcal{G}_{\Sigma}$ and a rule $r$ as above. A renaming $\mu$ is a match (of $r$ to $\gamma$ ) if $\boldsymbol{A}^{\mu}=\boldsymbol{A}^{\prime}$ and $X(\gamma) \cap X\left(\alpha^{\mu}\right) \subseteq X\left(\boldsymbol{A}^{\mu}\right) .{ }^{3}$ If additionally

\footnotetext{
${ }^{3}$ This condition makes sure that all nodes that are introduced on the right-hand side of a rule are renamed so that they are distinct from all nodes that do already occur in the graph. See also the discussion in Example 2.6, derivation (2.2).
} 
$\beta^{\prime} \in \mathcal{G}_{\mathcal{T}}$, then a match $\mu$ of $r$ derives $\gamma$ to the graph $\gamma^{\prime}=\beta \alpha^{\mu} \beta^{\prime}$. This is denoted as $\gamma \Rightarrow_{r, \mu} \gamma^{\prime}$, or just as $\gamma \Rightarrow_{r} \gamma^{\prime}$. We write $\gamma \Rightarrow_{\mathcal{R}} \gamma^{\prime}$ if $\gamma \Rightarrow_{r} \gamma^{\prime}$ for some rule $r$ taken from a set $\mathcal{R}$ of rules.

A hyperedge replacement grammar $\Gamma=(\Sigma, \mathcal{T}, \mathcal{R}, Z)$ (HR grammar for short) consists of finite alphabets $\Sigma, \mathcal{T}$ as above, a finite set $\mathcal{R}$ of rules over $\Sigma$, and a start symbol $Z \in \mathcal{N}$ of arity 0 . $\Gamma$ generates the language

$$
\mathcal{L}(\Gamma)=\left\{g \in \mathcal{G}_{\mathcal{T}} \mid Z() \Rightarrow_{\mathcal{R}}^{*} g\right\}
$$

of terminal graphs. We call a graph $g$ valid with respect to $\Gamma$ if $\mathcal{L}(\Gamma)$ contains a graph $g^{\prime}$ with $g \bowtie g^{\prime}$.

Note that the definition of "derives" requires that $\beta^{\prime} \in \mathcal{G}_{\mathcal{T}}$. This means that we only consider rightmost derivations in this paper. As usual, by the contextfreeness of hyperedge replacement this does not imply any loss of generality. The reader should, however, bear in mind that "derivation" always means "rightmost derivation".

In the following, $\boldsymbol{Z}$ always denotes the literal $Z()$ of the start symbol of $\Gamma$. Moreover, we shall generally omit the subscript in $\Rightarrow_{\mathcal{R}}$ and $\Rightarrow_{\mathcal{R}}^{*}$, thus writing simply $\Rightarrow$ and $\Rightarrow^{*}$ instead because the HR grammar in question will always be clear from the context.

We call literals in $L i t_{\mathcal{T}}$ terminal and denote them as $\boldsymbol{a}, \boldsymbol{b}, \boldsymbol{c}, \ldots$, whereas nonterminal literals from $L_{i} t_{\mathcal{N}}$ are denoted as $\boldsymbol{A}, \boldsymbol{B}, \boldsymbol{C}, \ldots$. Terminal graphs, those in $\mathcal{G}_{\mathcal{T}}$, are denoted as $a, b, c, \ldots$, whereas graphs in $\mathcal{G}_{\Sigma}$, i.e., graphs that may contain nonterminal literals, are denoted as $\alpha, \beta, \gamma, \ldots$.

The next lemma follows directly from the definition of derivation steps by a straightforward induction on the length of the derivation.

Lemma 2.3. $\boldsymbol{Z} \Rightarrow^{*} \gamma$ implies $\boldsymbol{Z} \Rightarrow^{*} \gamma^{\varrho}$ for every renaming $\varrho$.

An even more immediate consequence of the definition of the derivation relation is the following:

Fact 2.4. For all graphs $\alpha, \alpha^{\prime}, \beta \in \mathcal{G}_{\Sigma}$ and $\beta^{\prime} \in \mathcal{G}_{\mathcal{T}}, \alpha \Rightarrow^{*} \alpha^{\prime}$ implies $\beta \alpha \beta^{\prime} \Rightarrow^{*}$ $\beta \alpha^{\prime} \beta^{\prime}$ if and only if $X\left(\alpha^{\prime}\right) \cap X\left(\beta \alpha \beta^{\prime}\right) \subseteq X(\alpha)$.

It is a well-known result [22, Theorem IV.4.1.2] that every HR grammar can be transformed into an equivalent reduced HR grammar where every nonterminal contributes to its language:

Definition 2.5 (Reduced HR Grammar). An HR grammar $\Gamma=(\Sigma, \mathcal{T}, \mathcal{R}$, $Z$ ) is reduced if, for every nonterminal literal $\boldsymbol{A} \in$ Lit $_{\mathcal{N}}, \boldsymbol{A} \neq \boldsymbol{Z}$, there are graphs $\alpha, \beta \in \mathcal{G}_{\Sigma}$ and $g \in \mathcal{G}_{\mathcal{T}}$ such that $\boldsymbol{Z} \Rightarrow^{*} \alpha \boldsymbol{A} \beta$ and $\boldsymbol{A} \Rightarrow^{*} g$.

Example 2.6 (Semantic Representation). An HR grammar can derive semantic representations of sentences of natural language. The semantic graphs in this example are much simplified Abstract Meaning Representations [3]. 
As in [15] (where the more powerful concept of contextual hyperedge replacement [10] is used), we represent the semantics of sentences using the predicates (i.e., verbs) 'persuade', 'try', and 'believe'. These yield interesting semantic graphs (to the extent such a small example reasonably can), because 'persuade' is an object control predicate (the patient of the persuasion is the agent of whatever she is persuaded to do) and 'try' is a subject control predicate (the agent of the trying is also the agent of whatever is being tried).

The represented patterns are

- " $x$ persuades $y$ to do $z$ "

- " $x$ tries to do $z$ "

- " $x$ believes $y$ "

- " $x$ believes $y$ about $z$ "

- " $x$ believes $y$ about himself"

The nodes of the graphs represent (anonymous) persons when they are leaves, and statements otherwise. Predicates are represented by terminal edges with the corresponding label and arity (with a further, first tentacle to the root of the statement governed by the predicate). The rules are as follows:

$$
\begin{aligned}
& Z() \rightarrow M(r, x) \\
& M(r, x) \rightarrow \operatorname{per}(r, x, y, z) M(z, y) \mid \operatorname{try}(r, x, z) M(z, x) \quad[p, t] \\
& \mid \begin{array}{l|l}
\operatorname{bel}(r, x, y) & \operatorname{bel}(r, x, y) M(y, z) \quad\left[b_{e}, b_{o}\right] \\
\operatorname{bel}(r, x, y) M(y, x) & {\left[b_{t}\right]}
\end{array}
\end{aligned}
$$

The graph $g$ representing the phrase " 1 persuades 4 to try to believe 6" can be derived in four steps; the matches $\mu_{1}$ to $\mu_{4}$ of the rules are given on the right):

$$
\begin{aligned}
& Z() \Rightarrow M(1,2) \\
& \underset{p}{\Rightarrow} \operatorname{per}(1,2,4,3) M(3,4) \\
& \underset{t}{\Rightarrow} \operatorname{per}(1,2,4,3) \operatorname{try}(3,4,5) M(5,4) \\
& \underset{b_{e}}{\Rightarrow} \operatorname{per}(1,2,4,3) \operatorname{try}(3,4,5) \operatorname{bel}(5,4,6) \\
& \mu_{1}=\{\boldsymbol{r} / 1, \boldsymbol{x} / 2\} \\
& \mu_{2}=\{r / 1, x / 2, \boldsymbol{y} / 4, \boldsymbol{z} / 3\} \\
& \mu_{3}=\{r / 3, x / 4, \boldsymbol{z} / 5\} \\
& \mu_{4}=\{r / 5, x / 4, \boldsymbol{y} / 6\}
\end{aligned}
$$

When a graph is rewritten with a rule, the nodes not occurring on its left-hand side (shown in bold in $\mu_{1}$ to $\mu_{4}$ ) have to be renamed to nodes that do not occur in that graph. E.g., in the third step, the graph $\gamma_{2}=\operatorname{per}(1,2,4,3) M(3,4)$ is rewritten with rule $t$ so that node $z$ is renamed to node 5 , which does not occur in $X\left(\gamma_{2}\right)=\{1,2,3,4\}$, or, as it is expressed by the condition in Def. 2.2, $X\left(\gamma_{2}\right) \cap X\left(\alpha_{t}^{\mu_{3}}\right) \subseteq X\left(\boldsymbol{A}_{t}^{\mu_{3}}\right)$, i.e., $\{1,2,3,4\} \cap\{3,4,5\} \subseteq\{3,4\}$. Mapping the "new" node $z$ of rule $t$ to node 3 would falsely match $z$ to a node in $\gamma_{2}$.

Since $g$ can be derived, so can $g^{\prime}=\operatorname{per}(1,6,2,3) \operatorname{try}(3,2,5)$ bel $(5,2,4)$, i.e., the node names in derivations are irrelevant (Lemma 2.3). Furthermore, while the graph $h=\operatorname{try}(3,4,5) \operatorname{per}(1,2,4,3)$ bel $(5,4,6)$ cannot be derived, it is valid 
for this grammar since $g \bowtie h$. Fig. 1 shows how the rules for $M$ and the graph $g$ are drawn as diagrams, a visually convenient notation that specifies them up to equivalence.

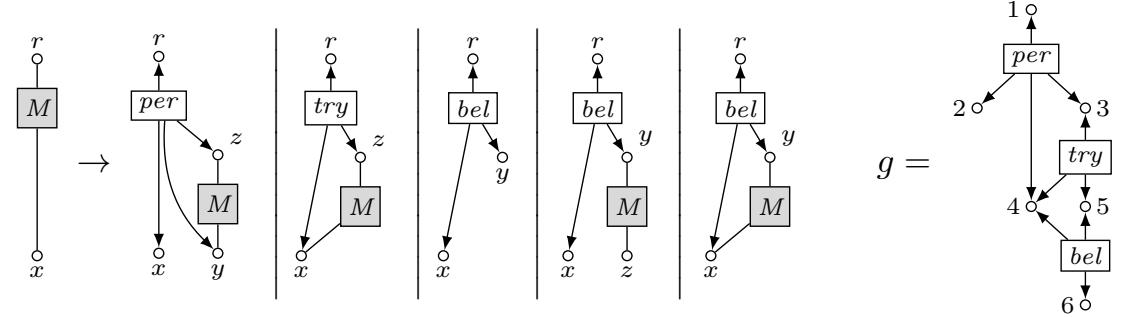

Figure 1: Diagrams of the rules in (2.1) and of the abstract meaning representation derived in (2.2). (Circles represent nodes, and boxes represent edges. The box of an edge contains its label, and is connected to the circles of its attached nodes by lines; these lines are ordered counter-clockwise around the edge, starting to its top. Names attached to nodes in rules define the correspondence between left-hand side and right-hand side. Vertical bars separate the right-hand sides of the rules for the nonterminal $M$.)

\section{Shift-Reduce Parsing of Strings}

The predictive shift-reduce parser for HR grammars borrows concepts known from the family of context-free $\mathrm{LR}(k)$ parsers for context-free string grammars [26], and extends them to the parsing of graphs. So we recall these concepts first. As context-free grammars, shift-reduce parsing, and $\operatorname{LR}(k)$ parsing can be found in every textbook on compiler construction, we discuss these matters just by means of a small example.

A Context-Free String Grammar for the Dyck Language. The Dyck language of matching nested square brackets "[" and "]" is generated by the context-free string grammar with the nonterminals $Z, T$, and $B$, and set of rules

$$
\mathcal{D}=\{Z \underset{0}{\rightarrow} T, T \underset{1}{\rightarrow}[B], B \underset{2}{\rightarrow} T B, B \underset{3}{\rightarrow} \varepsilon\},
$$

where $Z$ is the start symbol. A (rightmost) example derivation, where the replaced nonterminal is underlined in every step, is

$$
\underline{Z} \underset{0}{\Rightarrow} \underset{1}{\Rightarrow}[\underline{B}] \underset{2}{\Rightarrow}[T \underline{B}] \underset{3}{\Rightarrow}[\underline{T}] \underset{1}{\Rightarrow}[[\underline{B}]] \underset{3}{\Rightarrow}[[]] .
$$

A Nä̈ve Shift-Reduce Parser for the Dyck Grammar. A parser checks whether a string like "[[]]" belongs to the language of a grammar, and constructs a derivation if this is the case. A parser is formally defined as a stack automaton that reads an input string from left to right and uses its stack for remembering its moves. A shift-reduce parser is named after its two moves; it constructs a rightmost derivation in reverse. 
In a naïve shift-reduce parser, a configuration can be defined as $\alpha \cdot w \mid \bar{w}$, where the terminal string $w \bar{w}$ is the input (with the vertical bar indicating how far it has been read), and $\alpha$ is the stack, containing nonterminal and terminal symbols. The rightmost symbol of $\alpha$ is the top of the stack. Knowing the input, one of $w, \bar{w}$ can always be reconstructed from the other, so that we consider simplified configurations $\alpha \cdot w$ where the not yet read part of the input is omitted.

The parser performs the following types of moves (where $\alpha$ and $w$ are as explained above):

- Shift reads the first unread input symbol, ${ }^{4}$ and pushes it onto the stack. The parser for the Dyck language shifts square brackets:

$$
\alpha \cdot w \vdash \alpha[\cdot w[\quad \alpha \cdot w \vdash \alpha] \cdot w]
$$

- Reduce pops symbols from the stack if they form the right-hand side of a rule, and pushes its left-hand side onto it. Thus, in effect, it applies the rule in reverse. The parser for the Dyck language performs the following reductions:

$$
T \cdot w \underset{0}{\vdash} Z \cdot w \quad \alpha[B] \cdot w \vdash_{1} \alpha T \cdot w \quad \alpha T B \cdot w \vdash_{2} \alpha B \cdot w \quad \alpha \cdot w \vdash_{3} \alpha B \cdot w
$$

A successful parse that accepts a string $w$ is a sequence of moves starting from the initial configuration $\varepsilon \cdot \varepsilon$ to an accepting configuration $Z \cdot w$, as below:

$$
\begin{array}{rlllllllllll}
\varepsilon \cdot \varepsilon & \vdash & {[\cdot[} & \vdash & {[[\cdot[[} & \vdash & {[[B \cdot[[} & \vdash & {[[B] \cdot[[]} & \vdash & {[T \cdot[[]} \\
& \vdash & {[\underline{T B} \cdot[[]} & \vdash & {[B \cdot[[]} & \vdash & \underline{[B]} \cdot[[]] & \vdash & \underline{T} \cdot[[]] & \vdash & Z \cdot[[]]
\end{array}
$$

(The symbols replaced by reductions are underlined.) The reductions of a successful parse, read in reverse, yield a rightmost derivation, in this case the derivation (3.1) above.

The naïve shift-reduce parser is correct in the sense that a string has a successful parse if and only if it has a rightmost derivation.

Nondeterminism. The naïve parser is nondeterministic. E.g., in the configuration "[TB $\cdot[[]$ " above, the following moves are possible:

(i) a reduction by the rule $B \rightarrow T B$, leading to the configuration [B. [[];

(ii) a reduction by the rule $B \rightarrow \varepsilon$, leading to the configuration [TBB. [[]; and

(iii) a shift of an input symbol "[" or "]", leading to the configuration $[T B[\cdot[[][$ or $[T B] \cdot[[]]$.

\footnotetext{
${ }^{4}$ Since the unread input is omitted in our configurations, $a$ seems to come "out of thin air".
} 
Such a situation is called a conflict. Only move (i) will lead to a successful parse, namely the one above. If the naïve parser chooses the wrong move in such situations, it will have to backtrack, i.e., undo shifts and reductions and try alternative moves until it finds a successful parse, or fails altogether.

Backtracking makes parsing inefficient. To avoid this, the naïve shift-reduce parser can be refined by gathering information from the grammar that helps to predict which of its conflicting moves may lead to successful parses:

- The rules of a grammar allow to predict viable prefixes: these are sequences of terminal and nonterminal symbols that occur during rightmost derivations of terminal strings. In a successful parse, the stack of the parser always forms such a viable prefix. For the conflicts discussed above, the sequences " $[T B$ " and " $[B$ " occurring before and after move (i) are viable prefixes, whereas the sequences " $[T B B$ " and "[TB]" occurring after moves (ii) and (iii) are not. (such stacks cannot be reduced further.)

- A lookahead of the $k>0$ next input symbols may help to decide which move must be taken to make a parse successful. In the situation sketched above (where a lookahead of $k=1$ suffices), the reductions (i) and (ii) should only be made if the next input symbol is "]", which is the only terminal symbol that may follow $B$ in derivations with the grammar.

Several ways to determine viable prefixes, and different lengths of lookahead can be used to construct predictive shift-reduce parsers. A general one is Knuth's LR $(k)$ method [26]. Here we just consider the simplest case of DeRemer's $\operatorname{SLR}(k)$ parser [8], for a single symbol of lookahead, i.e., $k=1$.

Nondeterministic Characteristic Finite-State Automata. The viable prefixes of a context-free grammar form a regular language of nonterminal and terminal symbols that is generated by an automaton, known as characteristic finite-state automaton (CFA), which can be determined from the grammar as follows:

- The states of the CFA are so-called items, rules with an additional dot occurring in the right-hand side. The dot indicates how far parsing has proceeded. For instance, the rule $T \rightarrow[B]$ of the Dyck grammar leads to items $T \rightarrow \cdot[B], T \rightarrow[\cdot B], T \rightarrow[B$.$] , and T \rightarrow[B] .$.

- A state like $T \rightarrow \cdot[B]$, where the dot is before some symbol (terminal or nonterminal), has a transition under this symbol to the state where the dot is behind that symbol, here a transition under the terminal "[" to $T \rightarrow[\cdot B]$.

A state like $T \rightarrow[\cdot B]$, with the dot before a nonterminal, does furthermore have transitions under the empty string $\varepsilon$ to all items for that nonterminal in which the dot is before the first symbol of the right-hand side, e.g. to states $B \rightarrow . T B$ and $B \rightarrow \cdot \varepsilon$.

Fig. 2 shows the CFA for the Dyck grammar; it is nondeterministic, which is caused by its transitions under the empty string $\varepsilon$. Its start state $q_{0}$ (distinguished by the incoming edge without source node and label) represents the 


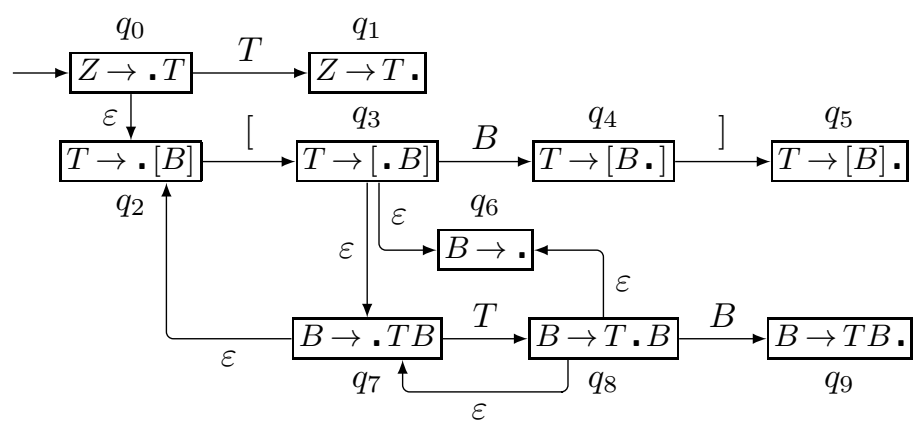

Figure 2: Nondeterministic characteristic finite-state automaton for the Dyck grammar

situation $Z \rightarrow . T$ where nothing has been recognized yet. A path from $q_{0}$ to some state $q$ in the CFA is an alternating sequence of states and labels of the transitions connecting them; the concatenations of the labels along such a path defines a string generated by the CFA. (Note that a path may contain states and labels repeatedly.)

Now a well-known result for shift-reduce parsing reads as follows: a string is generated by the CFA of a context-free grammar if and only if it is a viable prefix of a successful parse for that grammar. E.g., the viable prefixes "[TB" and " $[B$ " are generated by the CFA, whereas the sequences " $[T B B$ ", " $[T B[$ ", and " $[T B]$ " are not.

Deterministic Characteristic Finite-State Automata. The nondeterministic CFA of a context-free grammar is easy to define, but of limited practical use for parsing. Fortunately, it can be turned into a deterministic CFA for the same language (of viable prefixes). The well-known powerset construction works as follows: a state set $Q$ joins some state $q$ with all states $q^{\prime}$ reachable from $q$ by $\varepsilon$-transitions; $q$ is a kernel item of $Q$, whereas the $q^{\prime}$ are its closure items. Then the non- $\varepsilon$-transitions of the items in $Q$ have corresponding transitions to successor state sets $Q^{\prime}$ that again contain kernel and closure items. Thus

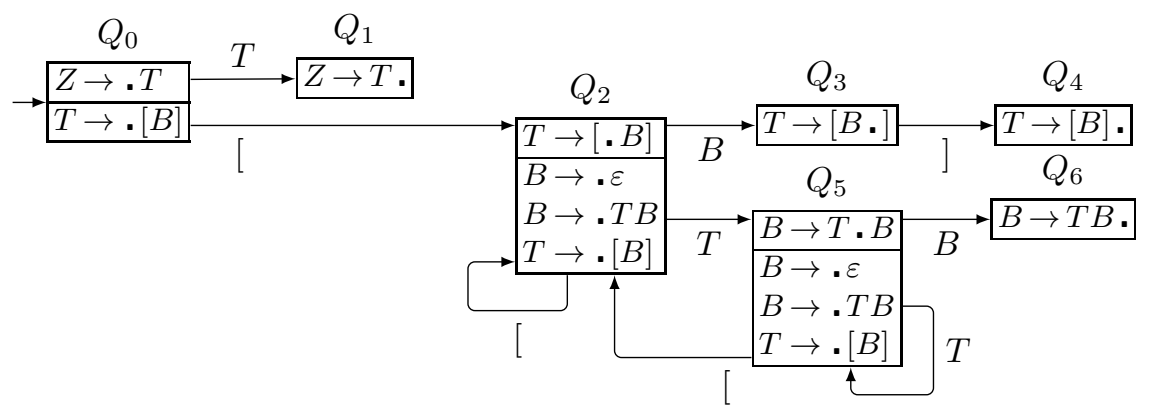

Figure 3: Deterministic characteristic finite-state automaton for the Dyck grammar 
state $q_{0}$ of the nondeterministic CFA is joined with state $q_{2}$ to form a state set $Q_{0}$, and states $q_{3}$ and $q_{8}$ are both joined with states $q_{6}, q_{7}$, and $q_{2}$ to form state sets $Q_{2}$ and $Q_{5}$, respectively, while the states $q_{1}, q_{4}, q_{5}$, and $q_{9}$ form singleton state sets $Q_{1}, Q_{3}, Q_{4}$, and $Q_{6}$ of the deterministic CFA. The transition diagram of the deterministic CFA for the Dyck grammar is shown in Fig. 3.

The powerset construction may let the number of states explode (yielding $2^{n}$ state sets for $n$ states of the nondetermistic CFA). However, this rarely occurs in practice; in our example, the number of states does even decrease.

SLR(1) Parsing. The stack of the SLR(1) parser is modified to contain a sequence like " $Q_{0}\left[Q_{2}\left[Q_{2} T Q_{5} B Q_{6}\right.\right.$ ", recording a path $Q_{0} \stackrel{[}{\rightarrow} Q_{2} \stackrel{[}{\rightarrow} Q_{2} \stackrel{T}{\rightarrow} Q_{5} \stackrel{B}{\rightarrow} Q_{6}$ in its deterministic CFA, starting in its initial state. The moves of the parser are determined by its current (topmost) state, and are modified in comparison to those of the nondeterministic parser as follows:

- Shift reads the next input symbol $a$ if the current state is $Q$ and the deterministic CFA contains a transition $Q \stackrel{a}{\rightarrow} Q^{\prime}$. The move pushes $a$ onto the stack, together with the successor state $Q^{\prime}$. For our grammar, and state numbers $i \in\{0,2,5\}$ :

$$
\alpha Q_{i} \cdot w \vdash \alpha Q_{i}\left[Q_{2} \cdot w\left[\quad \alpha Q_{3} \cdot w \vdash \alpha Q_{3}\right] Q_{4} \cdot w\right]
$$

- Reduce pops the right-hand side of a rule $A \rightarrow \beta$ (and the intermediate states) off the stack, leaving a state $Q$ on top, which has a transition $Q \stackrel{A}{\rightarrow} Q^{\prime}$. Then $A$ and $Q^{\prime}$ are pushed onto the stack. The $\operatorname{SLR}(1)$ parser performs a reduction only if the lookahead - the next input symbol - is a follower symbol of $A$. We write "if $\ell=a$ " to indicate that the required lookahead symbol is $a$. Then the reductions of the example grammar are as follows:

$$
\begin{array}{rccc}
\alpha Q_{0}\left[Q_{2} B Q_{3}\right] Q_{4} \cdot w & { }_{1} & \alpha Q_{0} T Q_{1} \cdot w & \\
\alpha Q_{2}\left[Q_{2} B Q_{3}\right] Q_{4} \cdot w & { }_{1} & \alpha Q_{2} T Q_{5} \cdot w & \\
\alpha Q_{5}\left[Q_{2} B Q_{3}\right] Q_{4} \cdot w & \vdash_{1} & \alpha Q_{5} T Q_{5} \cdot w & \\
\alpha Q_{2} T Q_{5} B Q_{6} \cdot w & \vdash & \alpha Q_{2} B Q_{3} \cdot w & \text { if } \ell=] \\
\alpha Q_{5} T Q_{5} B Q_{6} \cdot w & \vdash_{2} & \alpha Q_{5} B Q_{6} \cdot w & \text { if } \ell=] \\
\alpha Q_{2} \cdot w & \vdash & \alpha Q_{2} B Q_{3} \cdot w & \text { if } \ell=] \\
\alpha Q_{5} \cdot w & { }_{3} & \alpha Q_{5} B Q_{6} \cdot w & \text { if } \ell=]
\end{array}
$$

- The $\operatorname{SLR}(1)$ parser accepts a string $w$ if it reaches a configuration $\alpha Q_{1} \cdot w$.

The SLR(1) parser is correct as well: it recognizes the same language as the naïve shift-reduce parser. 
Conflicts. The deterministic CFA may reveal conflicts for SLR(1) parsing:

- If a state allows to shift some terminal $a$, and to reduce some rule under the same lookahead symbol $a$, this is a shift-reduce conflict.

- If a state allows reductions of different rules under the same lookahead symbol, this is a reduce-reduce conflict.

Whenever the automaton is conflict-free, the SLR(1) parser can choose its moves in a deterministic way.

The deterministic CFA for the Dyck grammar is indeed conflict-free: In states $Q_{2}$ and $Q_{5}$, rule $B \rightarrow \varepsilon$ can be reduced if the input begins with the only follower symbol "]" of $B$, which is not in conflict with the shift transitions from these states under the terminal "[". Reduce-reduce conflicts do not occur.

A deterministic parse with the SLR(1) parser is as follows:

$$
\begin{aligned}
& Q_{0} \cdot \varepsilon \quad \vdash \quad Q_{0}\left[Q _ { 2 } \cdot \left[\quad \vdash Q _ { 0 } \left[Q _ { 2 } \left[Q_{2} \cdot[[\right.\right.\right.\right. \\
& \vdash \quad Q_{0}\left[Q _ { 2 } \left[Q _ { 2 } B Q _ { 3 } \cdot \left[\left[\quad \vdash \quad Q _ { 0 } \left[Q_{2}\left[Q_{2} B Q_{3}\right] Q_{4} \cdot[[]\right.\right.\right.\right.\right. \\
& \stackrel{3}{\vdash} Q_{0}\left[Q _ { 2 } T Q _ { 5 } \cdot \left[[ ] \quad \vdash \quad Q _ { 0 } \left[Q_{2} T Q_{5} B Q_{6} \cdot[[]\right.\right.\right. \\
& \stackrel{-}{Q_{0}\left[Q_{2} B Q_{3} \cdot[[]\right.} \stackrel{3}{\vdash} Q_{0}\left[Q_{2} B Q_{3}\right] Q_{4} \cdot[[]] \\
& \vdash_{1} \quad Q_{0} T Q_{1} \cdot[[]]
\end{aligned}
$$

Note that the parser accepts the string [[]] because it reaches the accepting configuration $Q_{0} T Q_{1}$. [[]].

Each run of the deterministic parser corresponds to a run of the corresponding naïve shift-reduce parser (except the last move) when we ignore states and just consider the symbols on the stack. Thus the deterministic parser is correct, but it does only apply to grammars that are free of SLR(1) conflicts.

\section{A Naïve Shift-Reduce Parser for HR Grammars}

We now start to transfer the ideas of shift-reduce string parsing to HR grammars. In this section, we describe a naïve nondeterministic shift-reduce parser, which will be made more practical in the sections to follow. We prove the correctness of the naïve parser, i.e., that it can (nondeterministically) find a derivation for an input graph if and only if there is one.

Assumption 4.1. Throughout the rest of the paper, let $\Gamma=(\Sigma, \mathcal{T}, \mathcal{R}, Z)$ be the HR grammar for which we want to construct a parser. Without loss of generality, we assume that $\Gamma$ is reduced.

In the remainder of this paper, we will use a HR grammar generating trees as a running example.

Example 4.2 (HR Grammar for Trees). The HR grammar with start symbol $Z$ and the following rules derives $n$-ary trees.

$$
Z() \rightarrow \operatorname{root}(x) T(x) \quad T(y) \rightarrow T(y) e(y, z) T(z) \quad T(y) \rightarrow \varepsilon
$$


We shall refer to these rules by the number $1,2,3$. Note that the unique edge labeled root marks the root of the tree, which is the unique node at which any derivation of the tree has to start, and will thus also be the one where parsing has to start. The empty sequence $\varepsilon$ in the last rule is actually a short-hand for the graph $\langle\{y\}, \varepsilon\rangle$ consisting of a single node, rather than for the empty graph. Fig. 4 shows a derivation of the tree $t=\operatorname{root}(1) e(1,3) e(1,2) e(2,4)$. The tree $t^{\prime}=e(2,4) \operatorname{root}(1) e(1,3) e(1,2)$ is valid w.r.t. the grammar since $t^{\prime} \bowtie t$.

$$
\begin{aligned}
& Z() \Rightarrow_{1} \operatorname{root}(1) T(1) \\
& \Rightarrow_{2} \operatorname{root}(1) \overline{T(1)} e(1,2) T(2) \\
& \Rightarrow_{2} \operatorname{root}(1) T(1) e(1,2) \overline{T(2)} e(2,4) T(4) \\
& \Rightarrow_{3} \operatorname{root}(1) T(1) e(1,2) T(2) e(2,4) \\
& \Rightarrow_{3} \operatorname{root}(1) T(1) e(1,2) \overline{e(2,4)} \\
& \Rightarrow_{2} \operatorname{root}(1) \overline{T(1)} e(1,3) T(3) e(1,2) e(2,4) \\
& \Rightarrow_{3} \operatorname{root}(1) T(1) e(1,3) \overline{e(1,2)} e(2,4) \\
& \Rightarrow_{3} \operatorname{root}(1) \overline{e(1,3)} e(1,2) e(2,4)
\end{aligned}
$$

Figure 4: A derivation of a tree

The diagrams of the rules are shown in Fig. 5 , and a diagram of the tree $t$ is shown in Fig. 6.

A shift-reduce parser of graphs will also be modeled by a stack automaton that reads the literals of an input graph and uses a stack for remembering its moves. A configuration of the naïve shift-reduce parser could take the form $\gamma \cdot g \mid \bar{g}$, where the terminal graph $g \bar{g}$ is the input (with the vertical bar indicating how far it has been read), and $\gamma$ is the stack, containing nonterminal and terminal literals; the rightmost symbol of $\gamma$ is the top of the stack. However, we have to keep in mind that literals of $\bar{g}$ can be read in any order, not just from left to right as in string parsing.

In a parse of a certain input graph, the unread part $\bar{g}$ of the input can always be reconstructed from the read part $g$, which does not only identify the edges that have been read, but also that their attached nodes (and only those) have been read as well. So we omit the unread input $\bar{g}$ in configurations.

Definition 4.3 (Parser Configuration). A (shift-reduce parser) configuration $\gamma \cdot g$ consists of graphs $\gamma \in \mathcal{G}_{\Sigma}$ and $g \in \mathcal{G}_{\mathcal{T}}$. The former is the stack

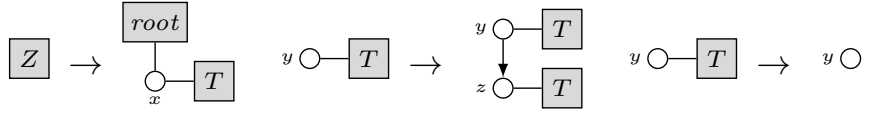

Figure 5: HR rules deriving trees. The binary terminal edge $e(y, z)$ is drawn as an arrow from node $y$ to node $z$.

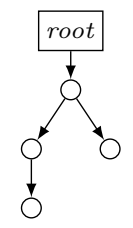

Figure 6: A tree 
whereas the latter is the already read subgraph of the input graph.

The parser begins with both the stack and the already read literals being empty, i.e., its initial configuration is $\varepsilon \cdot \varepsilon$. The parser then tries to reach an accepting configuration using shift and reduce moves, which are similar to the string case. Shift moves read literals of the input graph, which are then stored in the parser configuration. The parser accepts an input graph $g$ if it reaches a configuration where the stack consists just of the start graph $\boldsymbol{Z}$, and $g$ has been read completely. This situation is represented by an accepting configuration $\boldsymbol{Z} \cdot g^{\prime}$ with $g^{\prime} \bowtie g$. We will show in the following that reaching $\boldsymbol{Z} \cdot g^{\prime}$ means $\boldsymbol{Z} \Rightarrow^{*} g^{\prime} \bowtie g$, i.e., the parser has identified a permutation of the input graph literals that proves the validity of $g$ with respect to the grammar.

Definition 4.4 (Shift and Reduce Moves). A reduce move turns a configuration $\gamma \cdot g$ into $\gamma^{\prime} \cdot g$ if there is a graph $\alpha \in \mathcal{G}_{\Sigma}$, a rule $\boldsymbol{A} \rightarrow \varrho$ and a renaming $\mu$ such that $\gamma=\alpha \varrho^{\mu}, \gamma^{\prime}=\alpha \boldsymbol{A}^{\mu}$, and $X(\alpha) \cap X\left(\varrho^{\mu}\right) \subseteq X\left(\boldsymbol{A}^{\mu}\right)$. We write

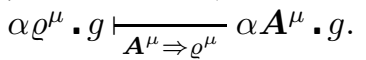

A shift move turns a configuration $\gamma \cdot g$ into $\gamma \boldsymbol{a} \cdot g \boldsymbol{a}$ for a literal $\boldsymbol{a} \in L_{i t}$ if $X(\boldsymbol{a}) \cap X(g) \subseteq X(\gamma)$. We write $\gamma \cdot g \vdash_{\text {sh }} \gamma \boldsymbol{a} \cdot g \boldsymbol{a}$.

We write $\gamma \cdot g \vdash \gamma^{\prime} \cdot g^{\prime}$ and call it a move of the parser if $\gamma \cdot g \vdash_{\text {sh }} \gamma^{\prime} \cdot g^{\prime}$ or

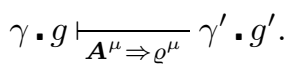

Let us briefly discuss the difference between these shift and reduce moves on the one hand and their counterparts in string parsing on the other hand.

A shift move in string parsing always reads the first symbol of the remaining input; the string parser cannot choose the symbol to be shifted. The graph parser, in contrast, can pick any of the remaining (terminal) literals for a shift move, as long as the requirement $X(\boldsymbol{a}) \cap X(g) \subseteq X(\gamma)$ is satisfied. This adds another dimension of nondeterminism to the parsing of graphs.

A reduce move in string parsing replaces the right-hand side of a rule on the stack by its left hand side without further consideration. The graph parser, in contrast, must first rename the nodes in the rule (cf. Def. 2.2). The condition $X(\alpha) \cap X\left(\varrho^{\mu}\right) \subseteq X\left(\boldsymbol{A}^{\mu}\right)$ makes sure that $\gamma^{\prime}=\alpha \boldsymbol{A}^{\mu} \Rightarrow \alpha \varrho^{\mu}=\gamma$, i.e., the application of rule $\boldsymbol{A} \rightarrow \varrho$ to $\gamma^{\prime}$ indeed yields $\gamma$, and so the move is a correct backwards application of the rule. In other words, replacing the literals of $\varrho^{\mu}$ by $\boldsymbol{A}^{\mu}$ also removes all nodes from $\gamma$ that are generated by the derivation step $\gamma^{\prime} \Rightarrow \gamma$. The requirement $X(\boldsymbol{a}) \cap X(g) \subseteq X(\gamma)$ of shift moves eventually checks that these nodes do not occur in literals still to be processed. Note that if a literal violates the condition for a shift move once, it will never satisfy this condition, and will thus never be shifted. Once a condition for a shift move fails for any of the remaining literals, the parse will eventually fail altogether.

Example 4.5 (Naïve Shift-Reduce Parser for Trees). The naïve shift-reduce parser for the tree-generating grammar of Example 4.2 has the following operations: 
- shift operations for the edges labeled with root and $e$, and

- reductions for the tree-generating rules.

Fig. 7 shows the moves of the naïve shift-reduce parser recognizing the tree $t$ with $t \bowtie \operatorname{root}(1) e(1,2) e(1,3) e(2,4)$. In many steps of this parse, the parser could make alternative decisions that may lead into a dead end:

1. In the first step, the parser could have shifted edge $e(1,3)$ instead of $\operatorname{root}(1)$; however, this would lead to a dead end.

2 . In the third step, the parser shifts the edge $e(1,2)$; it could have chosen $e(1,3)$ instead, which is another match of the edge pattern $e(y, z)$. In this case the parser could succeed anyway, arriving at the graph $g^{\prime}=$ $\operatorname{root}(1) e(1,3) e(1,2) e(2,4)$. However, it holds that $g \bowtie g^{\prime}$.

Shifting $e(2,4)$ is also possible in this step, but would lead to failure.

3 . In step four, the parser could shift edge $e(2,4)$ instead of reducing rule 3 . This choice of a shift instead of a reduction would also cause failure.

4. Instead of reducing $T(2) e(2,4) T(4)$ to $T(2)$ in the seventh step, the parser could reduce by rule 3 . Reduction of a rule like $T(y) \rightarrow \varepsilon$ is possible in every step. Here it would also lead to a dead end.

\begin{tabular}{|c|c|c|}
\hline & $\begin{array}{c}\varepsilon \cdot \varepsilon \\
\operatorname{root}(1) \cdot \operatorname{root}(1)\end{array}$ & \\
\hline & $\operatorname{root}(1) T(1) \cdot \operatorname{root}(1)$ & $y / 1$ \\
\hline & $\operatorname{root}(1) T(1) e(1,2) \cdot \operatorname{root}(1) e(1,2)$ & \\
\hline & $\operatorname{root}(1) T(1) e(1,2) T(2) \cdot \operatorname{root}(1) e(1,2)$ & $y / 2$ \\
\hline & $\operatorname{root}(1) T(1) e(1,2) T(2) e(2,4) \cdot \operatorname{root}(1) e(1,2) e(2,4)$ & \\
\hline & $\operatorname{root}(1) T(1) e(1,2) T(2) e(2,4) T(4) \cdot \operatorname{root}(1) e(1,2) e(2,4)$ & $y / 4$ \\
\hline & $\operatorname{root}(1) \underline{T(1) e(1,2) T(2)} \cdot \operatorname{root}(1) e(1,2) e(2,4)$ & $y / 2, z / 4$ \\
\hline & $\operatorname{root}(1) T(1) \cdot \operatorname{root}(1) e(1,2) e(2,4)$ & $y / 1, z / 2$ \\
\hline & $\operatorname{root}(1) T(1) e(1,3) \cdot \operatorname{root}(1) e(1,2) e(2,4) e(1,3)$ & \\
\hline & $\operatorname{root}(1) T(1) e(1,3) T(3) \cdot \operatorname{root}(1) e(1,2) e(2,4) e(1,3)$ & $y / 3$ \\
\hline & $\operatorname{root}(1) T(1) \cdot \operatorname{root}(1) e(1,2) e(2,4) e(1,3)$ & $y / 1, z / 3$ \\
\hline & $\overline{Z Z()} \cdot \operatorname{root}(1) e(1,2) e(2,4) e(1,3)$ & $x / 1$ \\
\hline
\end{tabular}

Figure 7: Moves of the naïve shift-reduce parser when recognizing the tree in Example 4.2. Places on the stack where reductions occur are underlined. Matches for rules in reductions appear in the rightmost column.

We now show that a parse consisting of shift and reduce moves corresponds to a derivation and vice versa. We first show that each parse yields a derivation (Lemma 4.6) and then that each derivation yields a parse (Lemma 4.8). 
Lemma 4.6. For every sequence $\gamma \cdot g \vdash^{*} \gamma^{\prime} \cdot g^{\prime}$ of moves with $X(\gamma) \subseteq X(g)$, there is a graph $u \in \mathcal{G}_{\mathcal{T}}$ such that $g^{\prime}=g u$ and $\gamma^{\prime} \Rightarrow^{*} \gamma u$. Moreover, $X\left(\gamma^{\prime}\right) \subseteq$ $X\left(g^{\prime}\right)$.

Proof. Let $\gamma \cdot g \vdash^{n} \gamma^{\prime} \cdot g^{\prime}$ be any sequence of moves with $X(\gamma) \subseteq X(g)$. We prove the statement by induction on $n$. For $n=0$ it holds with $u=\varepsilon$.

For $n>0$ and the last move being a shift move, the sequence has the form

$$
\gamma \cdot g \vdash^{n-1} \gamma^{\prime \prime} \cdot g^{\prime \prime} \vdash_{\text {sh }} \gamma^{\prime \prime} \boldsymbol{a} \cdot g^{\prime \prime} \boldsymbol{a}=\gamma^{\prime} \cdot g^{\prime}
$$

for some $\boldsymbol{a} \in \operatorname{Lit}_{\mathcal{T}}, g^{\prime \prime} \in \mathcal{G}_{\mathcal{T}}$, and $\gamma^{\prime \prime} \in \mathcal{G}_{\Sigma}$. By the induction hypothesis, there is a graph $u^{\prime} \in \mathcal{G}_{\mathcal{T}}$ such that $g^{\prime \prime}=g u^{\prime}$ and $\gamma^{\prime \prime} \Rightarrow^{*} \gamma u^{\prime}$. Let $u=u^{\prime} \boldsymbol{a}$. Since $X(\gamma) \subseteq$ $X(g)$, the definition of shift moves yields $X(\boldsymbol{a}) \cap X\left(\gamma u^{\prime}\right) \subseteq X(\boldsymbol{a}) \cap X\left(g u^{\prime}\right)=$ $X(\boldsymbol{a}) \cap X\left(g^{\prime \prime}\right) \subseteq X\left(\gamma^{\prime \prime}\right)$. Therefore, by Fact $2.4, \gamma^{\prime}=\gamma^{\prime \prime} \boldsymbol{a} \Rightarrow^{*} \gamma u^{\prime} \boldsymbol{a}=\gamma u$ and $g^{\prime}=g^{\prime \prime} \boldsymbol{a}=g u^{\prime} \boldsymbol{a}=g u$.

For $n>0$ and the last move being a reduce move, the sequence has the form

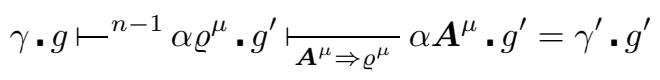

for a rule $r=\boldsymbol{A} \rightarrow \varrho$ and a renaming $\mu$. By the induction hypothesis, there is a graph $u \in \mathcal{G}_{\mathcal{T}}$ such that $g^{\prime}=g u$ and $\alpha \varrho^{\mu} \Rightarrow^{*} \gamma u$, and by the definition of reduce moves, $X(\alpha) \cap X\left(\varrho^{\mu}\right) \subseteq X\left(\boldsymbol{A}^{\mu}\right)$. Therefore, $\gamma^{\prime}=\alpha \boldsymbol{A}^{\mu} \Rightarrow \alpha \varrho^{\mu} \Rightarrow^{*} \gamma u$.

Finally, $X\left(\gamma^{\prime}\right) \subseteq X(\gamma u) \subseteq X(g u)=X\left(g^{\prime}\right)$ as $\gamma^{\prime} \Rightarrow^{*} \gamma u$ and $X(\gamma) \subseteq X(g)$.

The following lemma is needed in the proof of Lemma 4.8; it generalizes the condition for applying one shift move to sequences of shift moves:

Lemma 4.7. $X(g) \cap X(u) \subseteq X(\gamma)$ implies $\gamma \cdot g \vdash_{\text {sh }} * \gamma u$. gu for all graphs $\gamma \in \mathcal{G}_{\Sigma}$ and $g, u \in \mathcal{G}_{\mathcal{T}}$.

Proof. We prove the lemma by induction on $n=|u|$. For $n=0$ it follows from $u=\varepsilon$. For $n>0$, let $g, u, \gamma$ as in the lemma, $u=\boldsymbol{a} u^{\prime}$ for some $\boldsymbol{a} \in L_{i t_{\mathcal{T}}}$ and $u^{\prime} \in \mathcal{G}_{\mathcal{T}}$. Then, $X(g) \cap X(\boldsymbol{a}) \subseteq X(g) \cap X(u) \subseteq X(\gamma)$, and therefore $\gamma \cdot g \vdash_{\text {sh }} \gamma \boldsymbol{a} \cdot g \boldsymbol{a}$. Further, $X(g \boldsymbol{a}) \cap X\left(u^{\prime}\right)=\left(X(g) \cap X\left(u^{\prime}\right)\right) \cup\left(X(\boldsymbol{a}) \cap X\left(u^{\prime}\right)\right) \subseteq$ $X(\gamma) \cup X(\boldsymbol{a})=X(\gamma \boldsymbol{a})$, which satisfies the condition of the lemma, hence $\gamma \boldsymbol{a} \cdot g \boldsymbol{a} !_{\mathrm{sh}}{ }^{*} \gamma \boldsymbol{a} u^{\prime} \cdot g \boldsymbol{a} u^{\prime}=\gamma u \cdot g u$ by the induction hypothesis.

Lemma 4.8. $\gamma \Rightarrow^{*} g$ implies $\varepsilon \cdot \varepsilon \vdash^{*} \gamma \cdot g$ for all graphs $\gamma \in \mathcal{G}_{\Sigma}$ and $g \in \mathcal{G}_{\mathcal{T}}$.

Proof. Let $\gamma \Rightarrow^{n} g$ be any derivation as in the lemma. We proceed by induction on $n$.

For $n=0$, we have $\gamma=g$ and, by Lemma $4.7, \varepsilon \cdot \varepsilon \vdash_{\text {sh }} * g \cdot g=\gamma \cdot g$.

For $n>0$, the derivation must be of the form

$$
\gamma=\alpha \boldsymbol{A}^{\mu} v \Rightarrow \alpha \varrho^{\mu} v \Rightarrow^{n-1} u v=g
$$


for some $u, v \in \mathcal{G}_{\mathcal{T}}, \alpha \in \mathcal{G}_{\Sigma}$, rule $\boldsymbol{A} \rightarrow \varrho$, and renaming $\mu$. By $\alpha \varrho^{\mu} \Rightarrow^{n-1} u$ and the induction hypothesis,

$$
\varepsilon \cdot \varepsilon \vdash^{*} \alpha \varrho^{\mu} \cdot u .
$$

The first derivation step in (4.1) implies $X(\alpha) \cap X\left(\varrho^{\mu}\right) \subseteq X\left(\boldsymbol{A}^{\mu}\right)$, which enables the following reduce move:

$$
\alpha \varrho^{\mu} \cdot u \varlimsup_{\boldsymbol{A}^{\mu} \Rightarrow \varrho^{\mu}} \alpha \boldsymbol{A}^{\mu} \cdot u .
$$

The inclusion $X(u) \cap X(v) \subseteq X\left(\alpha \boldsymbol{A}^{\mu}\right)$ holds by (4.1) and Fact 2.4, and thus

$$
\alpha \boldsymbol{A}^{\mu} \cdot u{ }_{\text {sh }}^{*} \alpha \boldsymbol{A}^{\mu} v \cdot u v=\gamma \cdot g
$$

by Lemma 4.7 .

We have thus proved the correctness of the naïve shift-reduce parser:

Theorem 4.9. For each graph $h \in \mathcal{G}_{\mathcal{T}}, \varepsilon \cdot \varepsilon \vdash^{*} \boldsymbol{Z}$. $h$ if and only if $\boldsymbol{Z} \Rightarrow^{*} h$.

Proof. For the only-if direction, set $\gamma=g=\varepsilon, \gamma^{\prime}=Z$, and $g^{\prime}=h$ in Lemma 4.6, and for the if direction, set $\gamma=\boldsymbol{Z}$ in Lemma 4.8.

Although the naïve parser is correct, it is not practically useful, as it is highly nondeterministic, which requires inefficient backtracking. In particular, it does not take into account the unread part of the input graph when selecting its next move. The following five sections are devoted to enhancements that make the naïve parser less nondeterministic and more efficient. We follow the ideas for shift-reduce string parsing described in Sect. 3:

1. In Sect. 5, we define viable prefixes of derivations, which are those graphs that may occur on the stack of successful parses.

2. In Sect. 6, we construct the nondeterministic characteristic finite automaton (CFA) of a grammar. The CFA generates all viable prefixes of the grammar; we say it approves them.

3. The nondeterministic CFA is then turned into an equivalent deterministic CFA, in Sect. 7.

4. In Sect. 8, the deterministic CFA is used to assist the naïve SR parser so that it only constructs viable prefixes of derivations. This makes sure that the parser can always continue a parse found so far, by some remaining input, but not necessarily by the remaining input of the current input graph. This is so because it does not "look ahead" into the unread part of the input graph when selecting its next move. An implementation would have to use backtracking when it runs into a dead end.

5. Finally, in Sect. 9, we describe how the parser can avoid backtracking by selecting its next move based on information from the unread part of the input graph. This corresponds to the lookahead in string parsing. And, likewise, this is not possible if the deterministic CFA has conflicts. For the HR grammar meeting these conditions, we can construct a predictive SR parser that "knows" the appropriate move in each situation, so that it does not need backtracking, and is efficient. 


\section{Viable Prefixes of Graphs}

The naïve shift-reduce parser can find a successful parse for every valid graph (and only for those), but it must choose the right moves to avoid backtracking. Bear in mind that the parser can always perform a shift move as long as the input graph has not yet been read in its entirety. In particular, all literals can be shifted right away. Also, an $\varepsilon$-rule (like $T(y) \rightarrow \varepsilon$ in Example 4.2) can always be reduced. This will typically lead into a dead end. We shall now distinguish stacks that may occur in successful parses from those that do not. This will eventually result in the characteristic finite automaton that "assists" the parser. We follow a similar line of argument as for string parsing and define so-called viable prefixes first [2, Sect. 5.3.2].

Assumption 5.1. For the remainder of the paper, we add to $\Gamma$ a new nonterminal Start of arity zero, and the rule $\boldsymbol{S t a r t} \rightarrow \boldsymbol{Z}$ with $\boldsymbol{S t a r t}=\operatorname{Start}()$. Thus, the derivations starting with Start are just those in the original grammar, but with an additional first step $\boldsymbol{S t a r t} \Rightarrow \boldsymbol{Z}$. Clearly, the generated language is independent of whether $Z$ or Start is considered to be the initial nonterminal.

A viable prefix is a prefix of a graph derivable from Start by a nonempty derivation, provided that this prefix does not extend past the right-hand side of the most recently applied rule. More formally:

Definition 5.2 (Viable Prefix). A graph $\gamma \in \mathcal{G}_{\Sigma}$ is called a viable prefix if there are graphs $\alpha, \beta \in \mathcal{G}_{\Sigma}$ as well as $z \in \mathcal{G}_{\mathcal{T}}$ and a literal $\boldsymbol{A} \in$ Lit $_{\mathcal{N}}$ such that Start $\Rightarrow^{*} \alpha \boldsymbol{A} z \Rightarrow \alpha \beta z$ and $\gamma$ is a prefix of $\alpha \beta$.

Example 5.3. We illustrate Def. 5.2 by using an initial segment of the derivation in Fig. 4 (though now beginning with Start()):

$$
\begin{aligned}
\operatorname{Start}() & \Rightarrow * \overbrace{\operatorname{root}(1)}^{\alpha} \overbrace{T(1)}^{\boldsymbol{A}} \overbrace{e(1,2) e(2,4)}^{z} \\
& \Rightarrow 2 \overbrace{\underbrace{\operatorname{root}(1)}_{\gamma} \overbrace{T(1) e(1,3) T(3)}^{\alpha}}^{\overbrace{e(1,2) e(2,4)}^{\alpha}} .
\end{aligned}
$$

The graph $\gamma=\operatorname{root}(1) T(1) e(1,3) T(3)$ is the longest viable prefix of the derived graph; all prefixes of $\gamma$ are viable as well. In contrast to that, the graph $\operatorname{root}(1) T(1) e(1,3) T(3) e(1,2)$ is not a viable prefix, because the edge $e(1,2)$ is "beyond" the part of the graph that is generated from nonterminal $T(1)$.

Before we show that the set of viable prefixes is just the set of all stacks occurring in successful parses, we need two technical lemmata. Lemma 5.4 states that the set of viable prefixes does not change if we add to Def. 5.2 the additional requirement that the suffix $v$ with $\gamma v=\alpha \beta$ is a terminal graph.

Lemma 5.4. A graph $\gamma \in \mathcal{G}_{\Sigma}$ is a viable prefix if and only if there are graphs $\alpha, \beta \in \mathcal{G}_{\Sigma}$ as well as $v, z \in \mathcal{G}_{\mathcal{T}}$ and a literal $\boldsymbol{A} \in$ Lit $_{\mathcal{N}}$ such that Start $\Rightarrow^{*}$ $\alpha \boldsymbol{A} z \Rightarrow \alpha \beta z=\gamma v z$. 
Proof. The if direction follows immediately from the definition of viable prefixes. For the only-if direction, let $\gamma$ be any viable prefix. Hence, there is a derivation

$$
\text { Start } \Rightarrow^{n} \alpha \boldsymbol{A} z \Rightarrow \alpha \beta z \Rightarrow^{*} g
$$

and $\alpha \beta=\gamma \delta$, for some $\delta \in \mathcal{G}_{\Sigma}$ and $g \in \mathcal{G}_{\mathcal{T}}$ (because we assume that $\Gamma$ is reduced). Without loss of generality, assume that the step $\alpha \boldsymbol{A} z \Rightarrow \alpha \beta z$ is the last one in this derivation such that $\gamma$ is a prefix of $\alpha \beta$. Then the next step (if there is one) replaces a nonterminal literal in $\gamma$, which means that $\delta z \in \mathcal{G}_{\mathcal{T}}$ (since all derivations are rightmost). Thus, the statement holds with $v=\delta$.

We now show that the set of viable prefixes is just the set of all stacks occurring in successful parses.

Lemma 5.5. For every sequence $\varepsilon \cdot \varepsilon \vdash^{*} \gamma \cdot g$ of moves such that $\gamma$ is a viable prefix, there is a graph $g^{\prime} \in \mathcal{G}_{\mathcal{T}}$ with $\gamma \cdot g \vdash^{*} \boldsymbol{Z} \cdot g g^{\prime}$.

Proof. Let $\varepsilon \cdot \varepsilon \vdash^{*} \gamma \cdot g$ be a sequence of moves as in the lemma. By Lemma 4.6, $\gamma \Rightarrow^{*} g$. We first show that there is a derivation

$$
\text { Start } \Rightarrow^{n} \alpha \boldsymbol{A} z \Rightarrow \alpha \beta z=\gamma v z \Rightarrow^{*} \text { gvz }
$$

where $v \in \mathcal{G}_{\mathcal{T}}$. Since $\gamma$ is a viable prefix and according to Lemma 5.4, there is a derivation $\boldsymbol{S t a r t} \Rightarrow^{n} \hat{\alpha} \hat{\boldsymbol{A}} \hat{z} \Rightarrow \hat{\alpha} \hat{\beta} \hat{z}$ for some $n \in \mathbb{N}$ such that $\gamma \hat{v}=\hat{\alpha} \hat{\beta}$ for a terminal graph $\hat{v} \in \mathcal{G}_{\mathcal{T}}$. However, one cannot conclude $\gamma \hat{v} \hat{z} \Rightarrow^{*} g \hat{v} \hat{z}$ because of possible name clashes. To circumvent this, we rename nodes that may cause such problems. For this purpose, choose any renaming $\mu$ with $\mu(x)=x$ if $x \in X(\gamma)$ and $\mu(x) \notin X(g)$ otherwise, and let $\alpha=\hat{\alpha}^{\mu}, \beta=\hat{\beta}^{\mu}, v=\hat{v}^{\mu}, z=\hat{z}^{\mu}$, and $\boldsymbol{A}=\hat{\boldsymbol{A}}$. By the choice of $\mu, \gamma=\gamma^{\mu}$ and $\gamma v=\alpha \beta$, as well as

$$
X(v z) \cap X(g) \subseteq X(\gamma) .
$$

Hence, $\gamma v z \Rightarrow^{*} g v z$ by Fact 2.4. By Lemma 2.3, we thus have a derivation as in (5.1). Note that

$$
X(\alpha) \cap X(\beta) \subseteq X(\boldsymbol{A})
$$

follows from $\alpha \boldsymbol{A} z \Rightarrow \alpha \beta z$.

We now show that, for every sequence $\varepsilon \cdot \varepsilon \vdash^{*} \gamma \cdot g$ of moves and every derivation (5.1), there is a sequence $\gamma \cdot g \vdash^{*} \boldsymbol{Z} \cdot g v z$ by induction on the length of the derivation in (5.1).

For $n=0$, we have $\alpha=z=\varepsilon$ and Start $\Rightarrow \beta=\boldsymbol{Z}=\gamma v$, i.e., $\gamma=\boldsymbol{Z}$ and $v=\varepsilon$. Hence, $\gamma \cdot g=\boldsymbol{Z} \cdot g v z \vdash^{0} \boldsymbol{Z} \cdot g v z$.

For $n>0$, we distinguish between two cases.

(1) The derivation (5.1) has the form

$$
\text { Start } \Rightarrow^{n-1} \delta \boldsymbol{B} u \Rightarrow \delta \varphi \boldsymbol{A} w u \Rightarrow \alpha \beta z=\gamma v z \Rightarrow^{*} g v z
$$


where $\alpha=\delta \varphi, z=w u$, and $\alpha \beta=\gamma v$. By (5.2), (5.3), and Lemma 4.7,

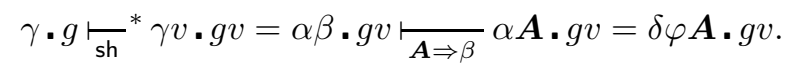

Note that (5.4) has the form of (5.1) where $\boldsymbol{B}$ plays the role of $\boldsymbol{A}, \delta \varphi \boldsymbol{A}$ the role of $\gamma, w$ the role of $v$, and $g v$ the role of $g$. Because of (5.5), we can make use of the induction hypothesis to conclude that $\delta \varphi \boldsymbol{A} \cdot g v \vdash^{*} \boldsymbol{Z} \cdot g v z$.

(2) The given derivation (5.1) has the form

$$
\text { Start } \Rightarrow^{n-1} \alpha \boldsymbol{A} u \boldsymbol{B} w \Rightarrow \alpha \boldsymbol{A} u v^{\prime} w \Rightarrow \alpha \beta z=\gamma v z \Rightarrow^{*} g v z
$$

with $\boldsymbol{B} \Rightarrow v^{\prime} \in \mathcal{G}_{\mathcal{T}}$ and $z=u v^{\prime} w$. By (5.2), (5.3), and Lemma 4.7,

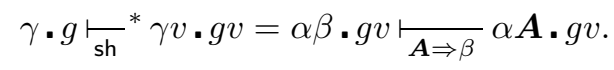

Note that (5.6) has the form of (5.1) where $\boldsymbol{B}$ plays the role of $\boldsymbol{A}, \alpha \boldsymbol{A}$ the role of $\gamma, u v^{\prime}$ the role of $v$, and $g v$ the role of $g$. Because of (5.7), we can use the induction hypothesis to conclude that $\alpha \boldsymbol{A} \cdot g v{ }^{*} \boldsymbol{Z} \cdot g v z$.

Lemma 5.6. For every sequence $\varepsilon \cdot \varepsilon \vdash^{*} \gamma \cdot u \vdash^{*} \boldsymbol{Z}$. uv of moves, $\gamma$ is a viable prefix.

Proof. If $\gamma=\boldsymbol{Z}$, then it is a viable prefix since $\boldsymbol{S t a r t} \Rightarrow \boldsymbol{Z}$. Otherwise, the sequence of moves has the form $\varepsilon \cdot \varepsilon \vdash^{*} \gamma \cdot u \vdash^{+} \boldsymbol{Z} \cdot u v$. As the last move is a reduce move, the sequence can be written as

$$
\varepsilon \cdot \varepsilon \vdash^{*} \gamma \cdot u{\leftarrow_{\mathrm{sh}}}^{*} \gamma v^{\prime} \cdot u v^{\prime}=\alpha \beta \cdot u v^{\prime} \varlimsup_{\boldsymbol{A} \Rightarrow \beta} \alpha \boldsymbol{A} \cdot u v^{\prime} \vdash^{*} \boldsymbol{Z} \cdot u v^{\prime} v^{\prime \prime}
$$

for graphs $v^{\prime}, v^{\prime \prime} \in \mathcal{G}_{\mathcal{T}}$ with $v=v^{\prime} v^{\prime \prime}$. Applying Lemma 4.6 to each subsequence yields $\boldsymbol{S t a r t} \Rightarrow \boldsymbol{Z} \Rightarrow^{*} \alpha \boldsymbol{A} v^{\prime \prime} \Rightarrow \alpha \beta v^{\prime \prime}=\gamma v^{\prime} v^{\prime \prime}$, i.e., $\gamma$ is a viable prefix.

An immediate consequence of Lemma 5.5 and Lemma 5.6 is the following:

Theorem 5.7. For every sequence $\varepsilon \cdot \varepsilon \vdash^{*} \gamma \cdot u$ of moves, there is a graph $v \in$ $\mathcal{G}_{\mathcal{T}}$ with $\gamma \cdot u \vdash^{*} \boldsymbol{Z}$. uv if and only if $\gamma$ is a viable prefix.

In other words, the stack of a reachable configuration is a viable prefix if and only if the naïve parser can reach the accepting configuration for some possible remaining sequence of literals.

\section{Nondeterministic Characteristic Finite-State Automata}

Let us now start to develop the means to "assist" the shift-reduce parser to restrict its moves to promising ones. The first step towards this goal is the construction of nondeterministic characteristic finite automata, defined next. 
Definition 6.1 (Nondeterministic CFA). The nondeterministic characteristic finite automaton (nCFA) for $\Gamma$ is the tuple $\mathfrak{A}=\left(Q, q_{0}, \Delta\right)$ consisting of the following components:

1. $Q=\{\boldsymbol{A} \rightarrow \alpha \cdot \beta \mid(\boldsymbol{A} \rightarrow \alpha \beta) \in \mathcal{R}\}$ is the finite set of states.

2. $q_{0}=($ Start $\rightarrow . \boldsymbol{Z})$ is the initial state.

3. $\Delta \subseteq Q \times\left(\right.$ Lit $\left._{\Sigma} \cup\{\varepsilon\}\right) \times Q$ is a ternary transition relation. Writing $p \stackrel{x}{\rightarrow} q$ if $(p, x, q) \in \Delta$, the transitions constituting $\Delta$ are:

(a) $(\boldsymbol{A} \rightarrow \alpha . \boldsymbol{l} \beta) \stackrel{\boldsymbol{l}}{\rightarrow}(\boldsymbol{A} \rightarrow \alpha \boldsymbol{l} . \beta)$ for every state $(\boldsymbol{A} \rightarrow \alpha . \boldsymbol{l} \beta) \in Q$, where $\boldsymbol{l} \in$ Lit $_{\Sigma} ;$ such a transition is called goto transition.

(b) $q \stackrel{\varepsilon}{\rightarrow} p$ for all states $q=(\boldsymbol{A} \rightarrow \alpha \cdot \boldsymbol{B} \beta) \in Q$ and $p=(\boldsymbol{C} \rightarrow \cdot \gamma) \in$ $Q$ such that $\ell(\boldsymbol{B})=\ell(\boldsymbol{C}) \in \mathcal{N}$; such a transition is called closure transition.

Assumption 6.2. Since we assume a fixed HR grammar $\Gamma$, we assume a fixed nCFA $\mathfrak{A}=\left(Q, q_{0}, \Delta\right)$ obtained from $\Gamma$ from now on.

Following the ideas discussed earlier, each item is a rule with a dot somewhere between literals in its right-hand side, indicating the division between literals that have already been processed and those which have not. Accordingly, the dot is moved across a literal when a corresponding literal is processed. We now start to formalize how an nCFA "approves" graphs (which will later turn out to be viable prefixes).

Intuitively, an nCFA approves a graph $\varphi$ if the sequence $\operatorname{lit}(\varphi)$ of literals corresponds to a sequence of state transitions, starting at the initial state. We define the notion of $n C F A$ configurations (or just configurations if it is clear from the context) to formalize this:

Definition 6.3 (nCFA Configuration). An $n C F A$ configuration $\varphi \diamond[q]^{\mu}$ consists of

- a graph $\varphi \in \mathcal{G}_{\Sigma}$

- a state $q=(\boldsymbol{A} \rightarrow \alpha \cdot \beta) \in Q$, and

- an injective partial function $\mu: X \rightarrow X$ with $X(\alpha) \subseteq \operatorname{dom}(\mu) \subseteq X(\alpha) \cup X(\boldsymbol{A})$.

The function $\mu$ in an nCFA configuration $\varphi \diamond[q]^{\mu}$ corresponds to the match defined in Def. 2.2; it maps rule nodes to nodes of the graph read so far. In an nCFA configuration, this match is not yet completely determined in general; the mapping of nodes that have not yet been read is still undefined. The mapping $\mu$ is extended when a literal is read, which means that all its attached nodes, if they have not been read as nodes of other literals earlier, are now read as well. As a consequence, nodes of state $q$ must be mapped by $\mu$ to nodes in $\varphi-$ unless they have not been read yet, in which case they are not in $\operatorname{dom}(\mu)$. Such nodes may only occur in literals behind the dot in $q$, which is reflected by the requirement that $X(\alpha) \subseteq \operatorname{dom}(\mu) \subseteq X(\alpha) \cup X(\boldsymbol{A})$. 
To compare literals that have only partially been matched, let $-\notin X$ be a special value denoting 'undefined'. Given a partial injective function $\mu: X \rightarrow X$ and a literal $\boldsymbol{l}=a\left(x_{1}, \ldots, x_{k}\right)$, we let $\boldsymbol{l}^{\mu}=a\left(y_{1}, \ldots, y_{k}\right)$ where, for all $1 \leq i \leq k$, $y_{i}=\mu\left(x_{i}\right)$ if $x_{i} \in \operatorname{dom}(\mu)$ and $y_{i}=-$ otherwise. Note that $\boldsymbol{l}^{\mu}$ is a literal if (and only if) $x_{1}, \ldots, x_{k} \in \operatorname{dom}(\mu)$. "Literals" which may contain '-' are called pseudo-literals. We let $X\left(\boldsymbol{l}^{\mu}\right)$ denote $\mu(X(\boldsymbol{l}))$. A slightly more general form of pseudo-literals will play an important role in Section 9.

An nCFA works by processing literals step by step while moving from state to state, represented by a corresponding sequence of nCFA configurations, starting at $\varepsilon \diamond\left[q_{0}\right]^{\iota}$, the initial configuration. Here, $\iota: X \rightarrow X$ is the totally undefined function with $\operatorname{dom}(\iota)=\varnothing$. Intuitively, $\varepsilon \diamond\left[q_{0}\right]^{\iota}$ being the initial configuration means that the nCFA starts with the empty graph $\varepsilon$ in $q_{0}=($ Start $\rightarrow . Z)$ and with no nodes mapped yet, the latter being indicated by the empty domain of $\iota$.

Each step of the nCFA is modeled by a move, defined as follows:

Definition 6.4 (nCFA Move). Let $\varphi \diamond[q]^{\mu}$ be a configuration. A goto transition $q \stackrel{l}{\rightarrow} q^{\prime}$ induces a goto move

$$
\varphi \diamond[q]^{\mu}{ }_{\text {I } \widetilde{g o}} \varphi l^{\nu} \diamond\left[q^{\prime}\right]^{\nu}
$$

where $\mu \sqsubseteq \nu, \operatorname{dom}(\nu)=\operatorname{dom}(\mu) \cup X(\boldsymbol{l})$, and $X\left(\boldsymbol{l}^{\nu}\right) \cap X(\varphi) \subseteq X\left(\boldsymbol{l}^{\mu}\right)$.

A closure transition $q \stackrel{\varepsilon}{\rightarrow} q^{\prime}$ with $q=(\boldsymbol{A} \rightarrow \alpha \cdot \boldsymbol{B} \beta)$ and $q^{\prime}=(\boldsymbol{C} \rightarrow \cdot \delta)$ induces a closure move

$$
\varphi \diamond[q]^{\mu}{ }_{\text {Icl }}^{\sim} \varphi \diamond\left[q^{\prime}\right]^{\nu}
$$

where $\boldsymbol{B}^{\mu}=\boldsymbol{C}^{\nu}$ and $\operatorname{dom}(\nu) \subseteq X(\boldsymbol{C})$.

We write $C$ I $\sim C^{\prime}$ if either $C$ I go $C^{\prime}$ or $C$ I $_{\text {cl }} C^{\prime}$, and call this a move.

A goto move applies a goto transition triggered by a (possibly nonterminal) literal $\boldsymbol{l}$. When the corresponding literal is processed, which also means that all its nodes have been read, all nodes of $\boldsymbol{l}$ are mapped by the resulting node mapping $\nu$. The processed literal is hence $\boldsymbol{l}^{\nu}$, which is added to the end of the approved graph, resulting in $\varphi \boldsymbol{l}^{\nu}$. The first two conditions, $\mu \sqsubseteq \nu$ and $\operatorname{dom}(\nu)=$ $\operatorname{dom}(\mu) \cup X(\boldsymbol{l})$, state that the mapping $\nu$ extends the previous mapping $\mu$ so as to map the entire literal $\boldsymbol{l}$. The remaining condition $X\left(\boldsymbol{l}^{\nu}\right) \cap X(\varphi) \subseteq X\left(\boldsymbol{l}^{\mu}\right)$ ensures that nodes that have already been read (i.e., those in $\varphi$ ) are not matched another time by extending $\mu$ to $\nu$.

A closure move applies a closure transition and corresponds to a derivation step, i.e., the mapping $\mu$ of nodes in $\boldsymbol{B}$ is translated into a mapping $\nu$ of the corresponding nodes in $\boldsymbol{C}$. Note that $\operatorname{dom}(\mu)$ and $\operatorname{dom}(\nu)$ are unrelated because the nodes in $\boldsymbol{B}$ and $\boldsymbol{C}$ may differ. Only nodes appearing in $\boldsymbol{C}$-but not necessarily all of them-are mapped by $\nu$; other nodes of state $q^{\prime}$ are not mapped because their corresponding nodes have not yet been read.

Definition 6.5. The nCFA approves a graph $\varphi \in \mathcal{G}_{\Sigma}$ if there is a configuration $C=\varphi \diamond[q]^{\mu}$ such that $\varepsilon \diamond\left[q_{0}\right]^{\iota}{ }^{1} \sim{ }^{*} C$. 


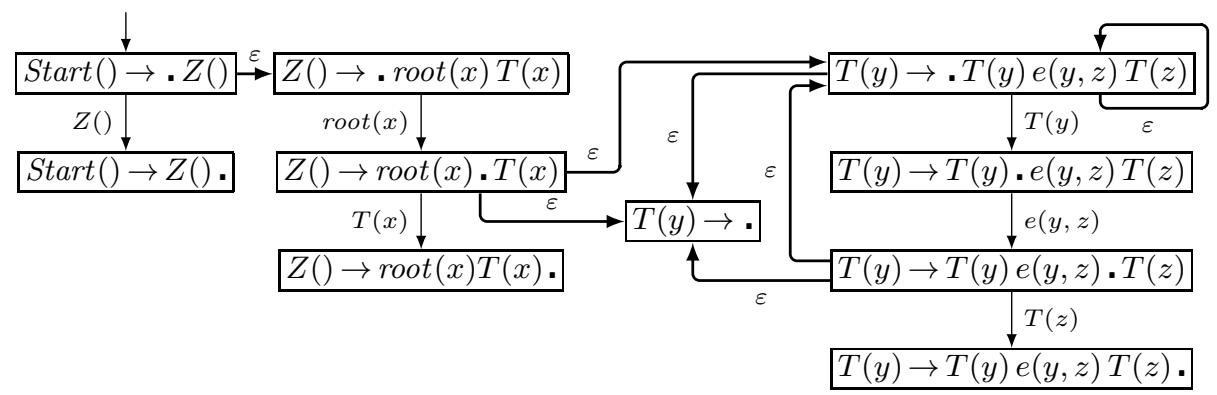

Figure 8: Nondeterministic CFA for the tree-generating grammar in Example 4.2. The initial state appears in the upper left. Closure transitions are drawn with thicker lines.

Example 6.6 (The nCFA for the Tree-Generating Grammar). Fig. 8 shows the transition diagram of the nondeterministic CFA for the treegenerating grammar in Example 4.2. In Fig. 9 we show moves of the nondeterministic CFA.

Every graph approved by the automaton is a viable prefix occurring in the derivation in Fig. 4 of Example 4.2, and in the parse shown in Fig. 7 of Example 4.5. We will show in the sequel that this is not a coincidence.

It is rather obvious that one can arbitrarily rename input graph nodes without affecting approval by the nCFA:

Fact 6.7. $\varepsilon \diamond\left[q_{0}\right]^{\iota} \mid \sim^{n} \varphi \diamond[q]^{\mu}$ implies $\varepsilon \diamond\left[q_{0}\right]^{\iota} \mid \sim^{n} \varphi^{\varrho} \diamond[q]^{\varrho \circ \mu}$ for every renaming $\varrho$.

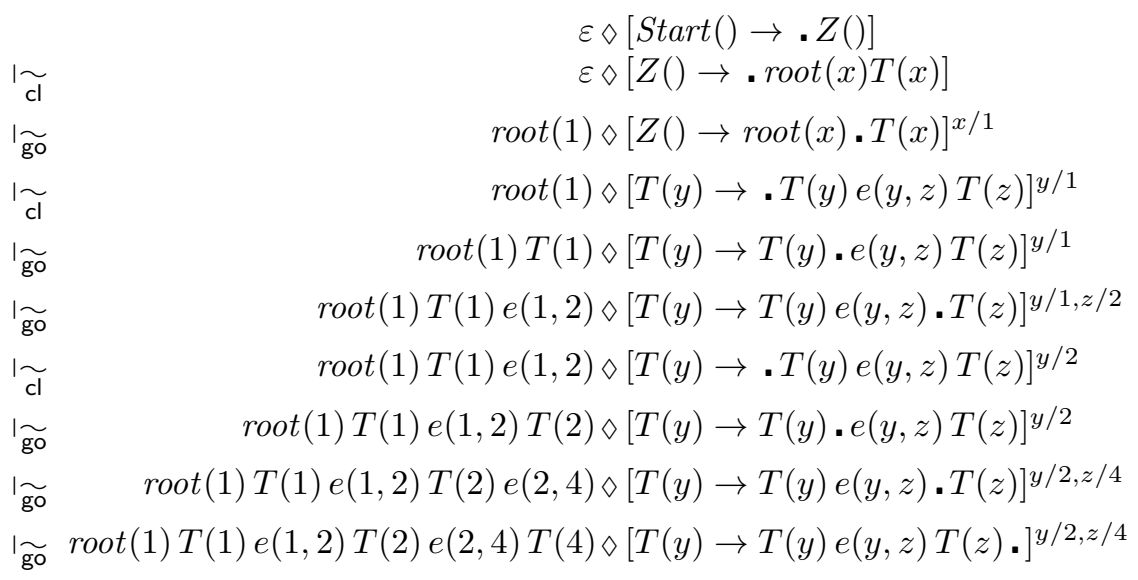

Figure 9: Approval of a graph with a nondeterministic CFA. Renamings $\mu$ of states $q_{i}$ with $\mu\left(x_{1}\right)=y_{1}, \ldots, \mu\left(x_{k}\right)=y_{k}$ are represented by exponents $x_{1} / y_{1}, \ldots, x_{k} / y_{k}$ 
Moreover, properties of goto moves can be generalized to goto sequences:

Lemma 6.8. $\varphi \diamond[\boldsymbol{A} \rightarrow \alpha \cdot \beta \gamma]^{\mu}{ }_{\text {I }{ }_{\text {go }} *}^{*} \varphi \diamond[\boldsymbol{A} \rightarrow \alpha \beta \cdot \gamma]^{\nu}$ implies $\mu \sqsubseteq \nu, \operatorname{dom}(\nu)=$ $\operatorname{dom}(\mu) \cup X(\beta)$, and $X(\varphi) \cap X\left(\alpha^{\nu} \beta^{\nu} \gamma^{\nu}\right) \subseteq X\left(\alpha^{\mu} \beta^{\mu} \gamma^{\mu}\right)$.

Proof. Consider any sequence $\varphi \diamond[\boldsymbol{A} \rightarrow \alpha \cdot \beta \gamma]^{\mu}{ }_{\text {| } \widetilde{\text { go }}}{ }^{n} \varphi \psi \diamond[\boldsymbol{A} \rightarrow \alpha \beta \cdot \gamma]^{\nu}$. We prove the lemma by induction on $n$. For $n=0$ it follows from $\mu=\nu$.

For $n>0$, we have the sequence

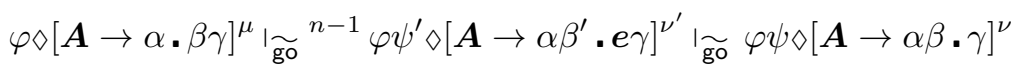

with $\beta=\beta^{\prime} \boldsymbol{e}$ and $\psi=\psi^{\prime} \boldsymbol{e}^{\nu}$. By the induction hypothesis and the definition of goto moves,

$$
\begin{aligned}
\mu & \sqsubseteq \nu^{\prime} \sqsubseteq \nu \\
X(\varphi) \cap X\left(\alpha^{\nu^{\prime}} \beta^{\nu^{\prime}} \gamma^{\nu^{\prime}}\right) & \subseteq X\left(\alpha^{\mu} \beta^{\mu} \gamma^{\mu}\right) \\
\operatorname{dom}(\nu) & =\operatorname{dom}\left(\nu^{\prime}\right) \cup X(\boldsymbol{e})=\operatorname{dom}(\mu) \cup X\left(\beta^{\prime}\right) \cup X(\boldsymbol{e}) \\
& =\operatorname{dom}(\mu) \cup X(\beta) \\
X\left(\boldsymbol{e}^{\nu}\right) \cap X\left(\varphi \psi^{\prime}\right) & \subseteq X\left(\boldsymbol{e}^{\nu^{\prime}}\right)
\end{aligned}
$$

By Def. 6.3 and (6.1), $X\left(\alpha^{\nu}\right)=X\left(\alpha^{\nu^{\prime}}\right)$ and $X\left(\beta^{\prime \nu}\right)=X\left(\beta^{\prime \nu^{\prime}}\right)$. Moreover, $X\left(\gamma^{\nu}\right) \subseteq X\left(\gamma^{\nu^{\prime}}\right) \cup X\left(\boldsymbol{e}^{\nu}\right)$ using (6.1) and (6.3), and $X\left(\boldsymbol{e}^{\nu}\right) \cap X(\varphi) \subseteq X\left(\boldsymbol{e}^{\nu^{\prime}}\right) \cap$ $X(\varphi)$ using (6.4). Therefore, $X(\varphi) \cap X\left(\alpha^{\nu} \beta^{\nu} \gamma^{\nu}\right) \subseteq X(\varphi) \cap X\left(\alpha^{\nu^{\prime}} \beta^{\nu^{\prime}} \gamma^{\nu^{\prime}}\right) \subseteq$ $X\left(\alpha^{\mu} \beta^{\mu} \gamma^{\mu}\right)$ using (6.2).

The following lemma shows that all images of nodes of the current nCFA state have been read already, i.e., occur in processed literals.

Lemma 6.9. $\varepsilon \diamond\left[q_{0}\right]^{\iota} \mid \sim^{*} \varphi \diamond[\boldsymbol{A} \rightarrow \alpha . \beta]^{\mu}$ implies $X\left(\alpha^{\mu} \beta^{\mu}\right) \subseteq X(\varphi)$.

Proof. We prove the lemma by induction on the length $n$ of the sequence of moves. For $n=0$ the inclusion follows from $\boldsymbol{A}=\boldsymbol{S t a r t}, \alpha=\varnothing, \beta=\boldsymbol{Z}$, and $\mu=\iota$.

For $n>0$ and the last move being a closure move, the sequence has the form

$$
\varepsilon \diamond\left[q_{0}\right]^{\iota} \sim^{n-1} \varphi \diamond[\boldsymbol{B} \rightarrow \delta \cdot \boldsymbol{C} \gamma]^{\nu}{ }_{\mathrm{cl}} \underset{\sim}{\sim} \varphi \diamond[\boldsymbol{A} \rightarrow \alpha \cdot \beta]^{\mu}
$$

with $\alpha=\varepsilon, \boldsymbol{C}^{\nu}=\boldsymbol{A}^{\mu}$, and $\operatorname{dom}(\mu) \subseteq X(\boldsymbol{A})$. Therefore, $X\left(\alpha^{\mu} \beta^{\mu}\right)=X\left(\boldsymbol{A}^{\mu}\right)=$ $X\left(\boldsymbol{C}^{\mu}\right) \subseteq X\left(\delta^{\nu} \boldsymbol{C}^{\nu} \gamma^{\nu}\right) \subseteq X(\varphi)$ using the induction hypothesis.

For $n>0$ and the last being a goto move, the sequence has the form

$$
\varepsilon \diamond\left[q_{0}\right]^{\iota} \sim^{n-1} \varphi^{\prime} \diamond\left[\boldsymbol{A} \rightarrow \alpha^{\prime} \cdot \boldsymbol{e} \beta\right]^{\nu} \text { । } \widetilde{\text { go }} \varphi \diamond[\boldsymbol{A} \rightarrow \alpha \cdot \beta]^{\mu}
$$


with $\alpha=\alpha^{\prime} \boldsymbol{e}, \varphi=\varphi^{\prime} \boldsymbol{e}^{\mu}, \nu \sqsubseteq \mu, \operatorname{dom}(\mu)=\operatorname{dom}(\nu) \cup X(\boldsymbol{e})$, and $X\left(\boldsymbol{e}^{\mu}\right) \cap X\left(\varphi^{\prime}\right) \subseteq$ $X\left(\boldsymbol{e}^{\nu}\right)$. Therefore, $X\left(\beta^{\mu}\right) \subseteq X\left(\beta^{\nu}\right) \cup X\left(\boldsymbol{e}^{\mu}\right)$ and

$$
\begin{aligned}
X\left(\alpha^{\mu} \beta^{\mu}\right) & \subseteq X\left(\alpha^{\prime \nu}\right) \cup X\left(\boldsymbol{e}^{\mu}\right) \cup X\left(\beta^{\nu}\right) \\
& \subseteq X\left(\alpha^{\prime \nu} \boldsymbol{e}^{\nu} \beta^{\nu}\right) \cup X\left(\boldsymbol{e}^{\mu}\right) \\
& \subseteq X\left(\varphi^{\prime}\right) \cup X\left(\boldsymbol{e}^{\mu}\right) \\
& =X(\varphi)
\end{aligned}
$$

using the induction hypothesis.

We now show that the graphs approved by the nCFA are viable prefixes (Lemma 6.10) and vice versa (Lemma 6.11).

Lemma 6.10. For every sequence

$$
\varepsilon \diamond\left[q_{0}\right]^{\iota} \sim^{*} \varphi \diamond[\boldsymbol{A} \rightarrow \alpha \cdot \beta]^{\mu}
$$

of moves and every injective function $\tau: X(\alpha \beta) \rightarrow X$ with $\mu \sqsubseteq \tau$ and $X\left(\beta^{\tau}\right) \cap$ $X(\varphi) \subseteq X\left(\beta^{\mu}\right)$, there exist $\psi \in \mathcal{G}_{\Sigma}$ and $z \in \mathcal{G}_{\mathcal{T}}$ such that

$$
\text { Start } \Rightarrow^{*} \psi \boldsymbol{A}^{\tau} z \Rightarrow \psi \alpha^{\tau} \beta^{\tau} z=\varphi \beta^{\tau} z .
$$

Proof. We proceed by induction on the number $n$ of moves. If $n=0$ the statement follows from the definition of initial nCFA configurations (with $\tau=\iota$, $\boldsymbol{A}=\boldsymbol{S t a r t}=\boldsymbol{S t a r t}^{\tau}, \varphi=\alpha=z=\varepsilon$, and $\beta=$ Start $)$.

If $n>1$ and the last move is a goto move, we have

$$
\varepsilon \diamond\left[q_{0}\right]^{\iota} \sim^{n-1} \varphi^{\prime} \diamond\left[\boldsymbol{A} \rightarrow \alpha^{\prime} \cdot \boldsymbol{e} \beta\right]^{\nu} \text { I } \widetilde{\text { go }} \varphi \diamond\left[\boldsymbol{A} \rightarrow \alpha^{\prime} \boldsymbol{e} \cdot \beta\right]^{\mu}=\varphi \diamond[\boldsymbol{A} \rightarrow \alpha \cdot \beta]^{\mu}
$$

where

$$
\begin{aligned}
\varphi & =\varphi^{\prime} \boldsymbol{e}^{\mu} \\
\nu & \sqsubseteq \mu \\
\operatorname{dom}(\mu) & =\operatorname{dom}(\nu) \cup X(\boldsymbol{e}) \\
X\left(\boldsymbol{e}^{\mu}\right) \cap X\left(\varphi^{\prime}\right) & \subseteq X\left(\boldsymbol{e}^{\nu}\right),
\end{aligned}
$$

Let $\tau$ be as in the lemma. Then $\nu \sqsubseteq \mu \sqsubseteq \tau$. To make use of the induction hypothesis, we additionally need that $X\left(\boldsymbol{e}^{\tau} \beta^{\tau}\right) \cap X\left(\varphi^{\prime}\right) \subseteq X\left(\boldsymbol{e}^{\nu} \beta^{\nu}\right)$. Indeed,

$$
\begin{aligned}
X\left(\boldsymbol{e}^{\tau} \beta^{\tau}\right) \cap X\left(\varphi^{\prime}\right) & =\left(X\left(\boldsymbol{e}^{\tau}\right) \cap X\left(\varphi^{\prime}\right)\right) \cup\left(X\left(\beta^{\tau}\right) \cap X\left(\varphi^{\prime}\right)\right) \\
& =\left(X\left(\boldsymbol{e}^{\mu}\right) \cap X\left(\varphi^{\prime}\right)\right) \cup\left(X\left(\beta^{\tau}\right) \cap X(\varphi) \cap X\left(\varphi^{\prime}\right)\right) \\
& \subseteq X\left(\boldsymbol{e}^{\nu}\right) \cup\left(X\left(\beta^{\mu}\right) \cap X\left(\varphi^{\prime}\right)\right) \\
& \subseteq X\left(\boldsymbol{e}^{\nu}\right) \cup X\left(\beta^{\nu}\right) \\
& =X\left(\boldsymbol{e}^{\nu} \beta^{\nu}\right) .
\end{aligned}
$$

Hence the induction hypothesis applies, yielding $\psi \in \mathcal{G}_{\Sigma}$ and $z \in \mathcal{G}_{\mathcal{T}}$ such that Start $\Rightarrow^{*} \psi \boldsymbol{A}^{\tau} z \Rightarrow \psi \alpha^{\prime \tau} \boldsymbol{e}^{\tau} \beta^{\tau} z=\varphi \beta^{\tau} z$, which proves the proposition. 
If $n>0$ and the last move is a closure move, we have

$$
\varepsilon \diamond\left[q_{0}\right]^{\iota}\left|\sim^{n-1} \varphi \diamond[\boldsymbol{B} \rightarrow \gamma \cdot \boldsymbol{C} \delta]^{\nu}\right| \sim \varphi \diamond[\boldsymbol{A} \rightarrow . \beta]^{\mu}
$$

where $\boldsymbol{C}^{\nu}=\boldsymbol{A}^{\mu}$, $\operatorname{dom}(\mu) \subseteq X(\boldsymbol{A})$, and $\alpha=\varepsilon$. Again, let $\tau: X(\alpha \beta) \rightarrow X$ be as in the statement of the lemma. To be able to use the induction hypothesis we need an injective function $\eta: X(\gamma \boldsymbol{C} \delta) \rightarrow X$ such that $\nu \sqsubseteq \eta$ and $X\left(\boldsymbol{C}^{\eta} \delta^{\eta}\right) \cap$ $X(\varphi) \subseteq X\left(\boldsymbol{C}^{\nu} \delta^{\nu}\right)$. But we also need $\boldsymbol{C}^{\eta}=\boldsymbol{A}^{\tau}$ in order to conclude a derivation Start $\Rightarrow^{*} \psi \boldsymbol{B}^{\eta} w \Rightarrow \psi \gamma^{\eta} \boldsymbol{C}^{\eta} \delta^{\eta} w=\varphi \boldsymbol{A}^{\tau} \delta^{\eta} w$. However, this may be impossible because of name clashes.

To solve this problem, we rename all nodes that may cause name clashes and use Fact 6.7. Choose any renaming $f$ with $f(x)=x$ for $x \in X(\varphi)$ and $f(x) \notin$ $X\left(\beta^{\tau}\right)$ for $x \in X\left(\beta^{\tau}\right) \backslash X(\varphi)$. Then $X\left(\boldsymbol{C}^{\nu}\right) \subseteq X(\varphi)$ because of Lemma 6.9, and hence $\boldsymbol{C}^{f \circ \nu}=\left(\boldsymbol{C}^{\nu}\right)^{f}=\boldsymbol{C}^{\nu}=\boldsymbol{A}^{\mu}$ as well as $\varphi^{f}=\varphi$, and therefore

$$
\varepsilon \diamond\left[q_{0}\right]^{l}\left|\sim^{n-1} \varphi \diamond[\boldsymbol{B} \rightarrow \gamma \cdot \boldsymbol{C} \delta]^{f \circ \nu}\right| \sim \varphi \diamond[\boldsymbol{A} \rightarrow \cdot \boldsymbol{\beta}]^{\mu} .
$$

We can now choose a renaming $\eta$ with $f \circ \nu \sqsubseteq \eta, X\left(\boldsymbol{C}^{\eta} \delta^{\eta}\right) \cap X(\varphi) \subseteq$ $X\left(\boldsymbol{C}^{f \circ \nu} \delta^{f \circ \nu}\right)$, and $\boldsymbol{C}^{\eta}=\boldsymbol{A}^{\tau}$, and by the induction hypothesis, Start $\Rightarrow^{*}$ $\psi \boldsymbol{B}^{\eta} w \Rightarrow \psi \gamma^{\eta} \boldsymbol{C}^{\eta} \delta^{\eta} w=\varphi \boldsymbol{A}^{\tau} \delta^{\eta} w$. Although $\boldsymbol{A}^{\tau} \Rightarrow \beta^{\tau}$, we cannot conclude $\varphi \boldsymbol{A}^{\tau} \delta^{\eta} w \Rightarrow \varphi \beta^{\tau} \delta^{\eta} w$ because $\delta^{\eta} w$ may contain nodes that are created by the derivation $\boldsymbol{A}^{\tau} \Rightarrow \beta^{\tau}$. Again, we solve this problem by renaming the nodes in $\delta^{\eta} w$ to new nodes. For this purpose, let $Y=X\left(\delta^{\eta} w\right) \backslash X\left(\varphi \boldsymbol{A}^{\tau}\right)$ and choose, for each $y \in Y$, a new node $n_{y} \in X \backslash X\left(\varphi \beta^{\tau} \delta^{\eta} w\right)$. Let $h$ be a renaming with $h(x)=n_{x}$ if $x \in Y$ and $h(x)=x$ for $x \in X\left(\varphi \beta^{\tau}\right)$. By Lemma 2.3, Start $\Rightarrow^{*}\left(\varphi \boldsymbol{A}^{\tau} \delta^{\eta} w\right)^{h}=\varphi \boldsymbol{A}^{\tau} \delta^{h \circ \eta} w^{h}$. By the definition of $h$, and because $\Gamma$ is reduced, there is a graph $u \in \mathcal{G}_{\mathcal{T}}$ such that

$$
\varphi \boldsymbol{A}^{\tau} \delta^{h \circ \eta} w^{h} \Rightarrow \varphi \beta^{\tau} \delta^{h \circ \eta} w^{h} \Rightarrow^{*} \varphi \beta^{\tau} u w^{h} .
$$

Therefore, Start $\Rightarrow^{*} \varphi \beta^{\tau} z$ for $z=u w^{h}$, which completes the proof.

Lemma 6.11. For every derivation $\boldsymbol{S t a r t} \Rightarrow^{*} \alpha \boldsymbol{A} z \Rightarrow \alpha \beta z$ and each prefix $\varphi$ of $\alpha \beta$, there is a sequence $\varepsilon \diamond\left[q_{0}\right]^{\nu} \sim^{*} \varphi \diamond[p]^{\nu}$ of moves (for a suitable state $[p]^{\nu}$ ).

Proof. We prove by induction on $n$ that Start $\Rightarrow^{n} \alpha \boldsymbol{A} z \Rightarrow \alpha \beta z$ implies $\varepsilon \diamond\left[q_{0}\right]^{\iota} \sim^{*} \varphi \diamond[p]^{\nu}$ for every prefix $\varphi$ of $\alpha \beta$.

For $n=0$, the derivation is of the form $\boldsymbol{S t a r t} \Rightarrow \boldsymbol{Z}=\beta$ and we have $\varphi=\varepsilon$ or $\varphi=\boldsymbol{Z}$. Hence, $\varphi \in\{\varepsilon, \boldsymbol{Z}\}$, and the proposition follows by making no move at all, or by making the goto move

$$
\varepsilon \diamond\left[q_{0}\right]^{\iota}{ }_{{ }_{\text {go }}} \boldsymbol{Z} \diamond[\text { Start } \rightarrow \boldsymbol{Z} \cdot]^{\iota} .
$$

For $n>0$, the initial part of the derivation up to $\alpha \boldsymbol{A} z$ has the form

$$
\text { Start } \Rightarrow^{n-1} \vartheta \boldsymbol{X} w \Rightarrow \vartheta \varrho w=\alpha \boldsymbol{A} z .
$$

There are two cases: 
(1) $\varrho \in \mathcal{G}_{\mathcal{T}}$.

Then $\vartheta=\alpha \boldsymbol{A} u$ for some $u \in \mathcal{G}_{\mathcal{T}}$, Start $\Rightarrow^{n-1} \alpha \boldsymbol{A} u \boldsymbol{X} w \Rightarrow \alpha \boldsymbol{A} u \varrho w \Rightarrow$ $\alpha \beta u \varrho w$. We distinguish two sub-cases:

(1a) $\varphi$ is a prefix of $\alpha$.

Then $\varphi$ is a prefix of $\alpha \boldsymbol{A} u \varrho$ and hence the claimed sequence is obtained directly from the induction hypothesis.

(1b) $\varphi=\alpha \tau$ for a prefix $\tau$ of $\beta$.

By the induction hypothesis, there is a sequence $\varepsilon \diamond\left[q_{0}\right]^{\iota} \mid \sim{ }^{m} \alpha \boldsymbol{A} \diamond[p]^{\nu}$ of moves. W.l.o.g, let $m$ be the minimum number of such moves. By Def. 6.4, this sequence must be of the form

$$
\varepsilon \diamond\left[q_{0}\right]^{\iota} \sim^{m-1} \alpha \diamond\left[\boldsymbol{B} \rightarrow \delta \cdot \boldsymbol{C} \delta^{\prime}\right]^{\mu}{ }_{\text {I go }} \alpha \boldsymbol{A} \diamond\left[\boldsymbol{B} \rightarrow \delta \boldsymbol{C} \cdot \delta^{\prime}\right]^{\nu}
$$

with $\boldsymbol{A}=\boldsymbol{C}^{\nu}$. Suppose that $\boldsymbol{A} \Rightarrow \beta$ uses rule $\boldsymbol{D} \rightarrow \psi \bar{\psi}$ with $|\tau|=|\psi|$. By making a closure move instead of the goto move at the end of the sequence above, we obtain

$$
\varepsilon \diamond\left[q_{0}\right]^{\iota}{ }^{\prime} \sim^{m-1} \alpha \diamond\left[\boldsymbol{B} \rightarrow \delta \cdot \boldsymbol{C} \delta^{\prime}\right]^{\mu}{ }_{\text {cl }} \alpha \diamond[\boldsymbol{D} \rightarrow \cdot \psi \bar{\psi}]^{\sigma}
$$

with $\boldsymbol{C}^{\mu}=\boldsymbol{D}^{\sigma}$. The claimed sequence is obtained by applying the appropriate number of goto moves:

$$
\alpha \diamond[\boldsymbol{D} \rightarrow \cdot \psi \bar{\psi}]^{\sigma}{ }_{\mathrm{g}_{\mathrm{go}}}{ }^{*} \alpha \tau \diamond[\boldsymbol{D} \rightarrow \psi \cdot \bar{\psi}]^{\sigma^{\prime}}=\varphi \diamond[\boldsymbol{D} \rightarrow \psi \cdot \bar{\psi}]^{\sigma^{\prime}} .
$$

(2) $\varrho \notin \mathcal{G}_{\mathcal{T}}$.

Then $\boldsymbol{A}$ is a literal in $\varrho$ and the given derivation has the form Start $\Rightarrow^{n-1}$ $\vartheta \boldsymbol{X} w \Rightarrow \vartheta \gamma \boldsymbol{A} u w \Rightarrow \vartheta \gamma \beta u w$ where $\vartheta \gamma=\alpha$, and thus $\varphi$ is a prefix of $\vartheta \gamma \beta$. We distinguish two sub-cases:

(2a) $\varphi$ is prefix of $\vartheta \gamma$.

As in case 1a, the proposition follows directly from the induction hypothesis because $\varphi$ is a prefix of $\vartheta \gamma \boldsymbol{A} u$.

(2b) $\varphi=\vartheta \gamma \tau$ for a prefix $\tau$ of $\beta$.

By the induction hypothesis, there is a sequence $\varepsilon \diamond\left[q_{0}\right]^{\iota} \mid \sim{ }^{*} \vartheta \gamma \boldsymbol{A} \diamond[p]^{\nu}$, and with a similar argument as in case $1 \mathrm{~b}, \varepsilon \diamond\left[q_{0}\right]^{\iota} \mid \sim^{*} \vartheta \gamma \tau \diamond\left[p^{\prime}\right]^{\sigma}$.

An immediate consequence of Lemma 6.10 and Lemma 6.11 is the following:

Theorem 6.12. A graph $\varphi \in \mathcal{G}_{\Sigma}$ is a viable prefix if and only if the $n C F A$ approves $\varphi$.

On the one hand, we have shown at the end of Sect. 4 that the naïve nondeterministic parser can reach the accepting configuration (with some appropriate remaining input) if and only if the current stack content is a viable prefix (Thm. 5.7). On the other hand, Thm. 6.12 shows that the nCFA approves precisely the viable prefixes. In other words, the naïve parser can avoid running into a situation in which no remaining input could ever make it accept, if moves are restricted to those which produce stacks approved by the nCFA. 


\section{Deterministic Characteristic Finite-State Automata}

Because of its spontaneously acting closure transitions, the nCFA cannot efficiently be used to improve the naïve shift-reduce parser by making sure that the stack of the parser is always a viable prefix. This is so because the nCFA, whenever it reaches a configuration, may also be in any configuration reachable by closure moves. In a deterministic implementation, all these configurations must be maintained simultaneously when the next goto move shall be made. To avoid this, we preprocess the nCFA and create the deterministic CFA (dCFA) by combining such simultaneously reachable states into new states, using a procedure similar to the classical powerset construction.

Literals of prefixes approved by an nCFA are images of literals of transitions and nCFA states under node mappings. The idea behind our adaptation of the traditional powerset construction to CFAs is to split such a node mapping into a composition of two mappings; the so-called parameter mapping is applied first, intuitively providing the node with a formal parameter name under which the node can be addressed. Later, the parameters are bound to nodes of the input graph by an input binding. Thus, the input binding maps parameters to nodes of the actual input graph during an actual run of the dCFA, whereas the parameter mapping is chosen when constructing the dCFA. Different nodes that are always mapped to the same input graph nodes and that belong to nCFA states combined in a common dCFA state, are mapped to the same parameter by the algorithm. For technical simplicity, we use nodes as parameters rather than introducing a special class of symbols for parameters. An item is then an nCFA state together with a parameter mapping:

Definition 7.1 (Item). An item $\langle q, \sigma\rangle$ consists of a state $q=(\boldsymbol{A} \rightarrow \alpha \cdot \beta)$ of our (fixed) nCFA and an injective partial parameter mapping $\sigma: X \rightarrow X$ with $X(\alpha) \subseteq \operatorname{dom}(\sigma) \subseteq X(\alpha) \cup X(\boldsymbol{A})$. $\mathcal{I}$ denotes the set of all items.

Note that the parameter mapping maps only those nodes of the item which occur in literals preceding the $\operatorname{dot}$ (or in $\boldsymbol{A}$ ).

If an nCFA processes a graph and reaches a state $q$, it can also reach those states reachable from $q$ by closure transitions. Of course, nodes must be renamed appropriately, as we are dealing with items instead of pure states. An item that is reachable from another item by a closure transition is called closure item of the latter. The formal definition reads as follows:

Definition 7.2 (Closure of Items). We call an item $\langle q, \tau\rangle$ a closure item of $\langle p, \sigma\rangle$, written $\langle p, \sigma\rangle \triangleright\langle q, \tau\rangle$, if $p=(\boldsymbol{A} \rightarrow \alpha \cdot \boldsymbol{B} \beta)$ and $q=(\boldsymbol{C} \rightarrow \cdot \delta), \boldsymbol{C}^{\tau}=\boldsymbol{B}^{\sigma}$, and $\operatorname{dom}(\tau) \subseteq X(\boldsymbol{C})$.

The closure of a set $I$ of items is the smallest set $J$ that contains all members of $I$ and, for each item in $J$, all its closure items.

The closure of a given set of items can be computed in the usual way by adding all closure items to the set and repeating this procedure as long as new items are added to the set: 
Fact 7.3. Let $J$ be the closure of a set I of items. Then, for every item $\left\langle q^{\prime}, \sigma^{\prime}\right\rangle \in$ $J$, there is an item $\langle q, \sigma\rangle \in I$ such that $\langle q, \sigma\rangle \triangleright^{*}\left\langle q^{\prime}, \sigma^{\prime}\right\rangle$.

In the definition of dCFAs below, states will be (finite) subsets $Q$ of $2^{\mathcal{I}}$. Such a set is said to be closed if it is its own closure, and its set of parameters is

$$
\operatorname{params}(Q)=\bigcup_{\langle q, \sigma\rangle \in Q} \sigma(X) .
$$

For a renaming $\mu$ with $\operatorname{dom}(\mu) \supseteq \operatorname{params}(Q)$, we let

$$
Q^{\mu}=\{\langle q, \mu \circ \sigma\rangle \mid\langle q, \sigma\rangle \in Q\}
$$

be the set of items obtained by renaming parameters according to $\mu$. Sets $Q, Q^{\prime} \subseteq$ $2^{\mathcal{I}}$ are equivalent, written $Q \approx Q^{\prime}$, if $Q^{\mu}=Q^{\prime}$ for some renaming $\mu$.

We are now ready to give the formal definition of dCFAs. Each dCFA state is a closed set of items. In particular, the initial state $Q_{0}$ is the closure of the initial state of the nCFA. Transitions in the dCFA are labeled with pairs that consist of a literal and a node mapping. The literal is the one that triggers the state transition; its nodes are parameters of the source state of the transition and new parameters whose "values" will be the corresponding nodes of the literal processed during parsing. The node mapping of the transition will later be used to set the "values" of the target state parameters.

Definition 7.4. A deterministic characteristic finite automaton (dCFA) $\mathfrak{C}=$ $\left(\mathcal{Q}, Q_{0}, \Delta\right)$ consists of a finite set $\mathcal{Q} \subseteq 2^{\mathcal{I}}$ of so-called $d C F A$ states, an initial state $Q_{0} \in \mathcal{Q}$, and a transition relation $\Delta$ with the following properties:

1. For all $Q, Q^{\prime} \in \mathcal{Q}, Q \approx Q^{\prime}$ implies $Q=Q^{\prime}$.

2. $Q_{0}$ is the closure of $\left\{\left\langle q_{0}, \iota\right\rangle\right\}$, where $q_{0}=(\boldsymbol{S t a r t} \rightarrow . \boldsymbol{Z})$ is the initial state of the nCFA.

3. $\Delta$ is a finite set of transition rules $Q \stackrel{(e, \mu)}{\longrightarrow} Q^{\prime}$, such that $Q, Q^{\prime} \in \mathcal{Q}$, $\boldsymbol{e} \in \operatorname{Lit}_{\Sigma}$, and $\mu: \operatorname{params}\left(Q^{\prime}\right) \rightarrow \operatorname{params}(Q) \cup X(\boldsymbol{e})$.

If it is obvious that we speak of the dCFA states of a dCFA, and thus there is no risk of confusing them with the concrete states to be defined soon, we may simply call them states.

Alg. 1 converts an nCFA into a corresponding dCFA. To determine the set of all transitions leaving a dCFA state $S$, it considers each element $\boldsymbol{l}$ of the set

$$
\text { leave }(S)=\bigcup_{\langle q, \sigma\rangle \in S} \text { leave }(q, \sigma)
$$

where leave $(\boldsymbol{A} \rightarrow \alpha \cdot, \sigma)=\varnothing$ and leave $(\boldsymbol{A} \rightarrow \alpha \cdot \boldsymbol{e} \beta, \sigma)=\left\{\boldsymbol{e}^{\sigma}\right\}$, i.e., leave $(S)$ contains mapped images of those literals that are labels of goto transitions leaving the corresponding nCFA states. These literals are mapped by the parameter mapping, i.e., they are in general pseudo-literals whose "nodes" are either parameters of $S$, or '-' if they are not (yet) mapped in $S$. 


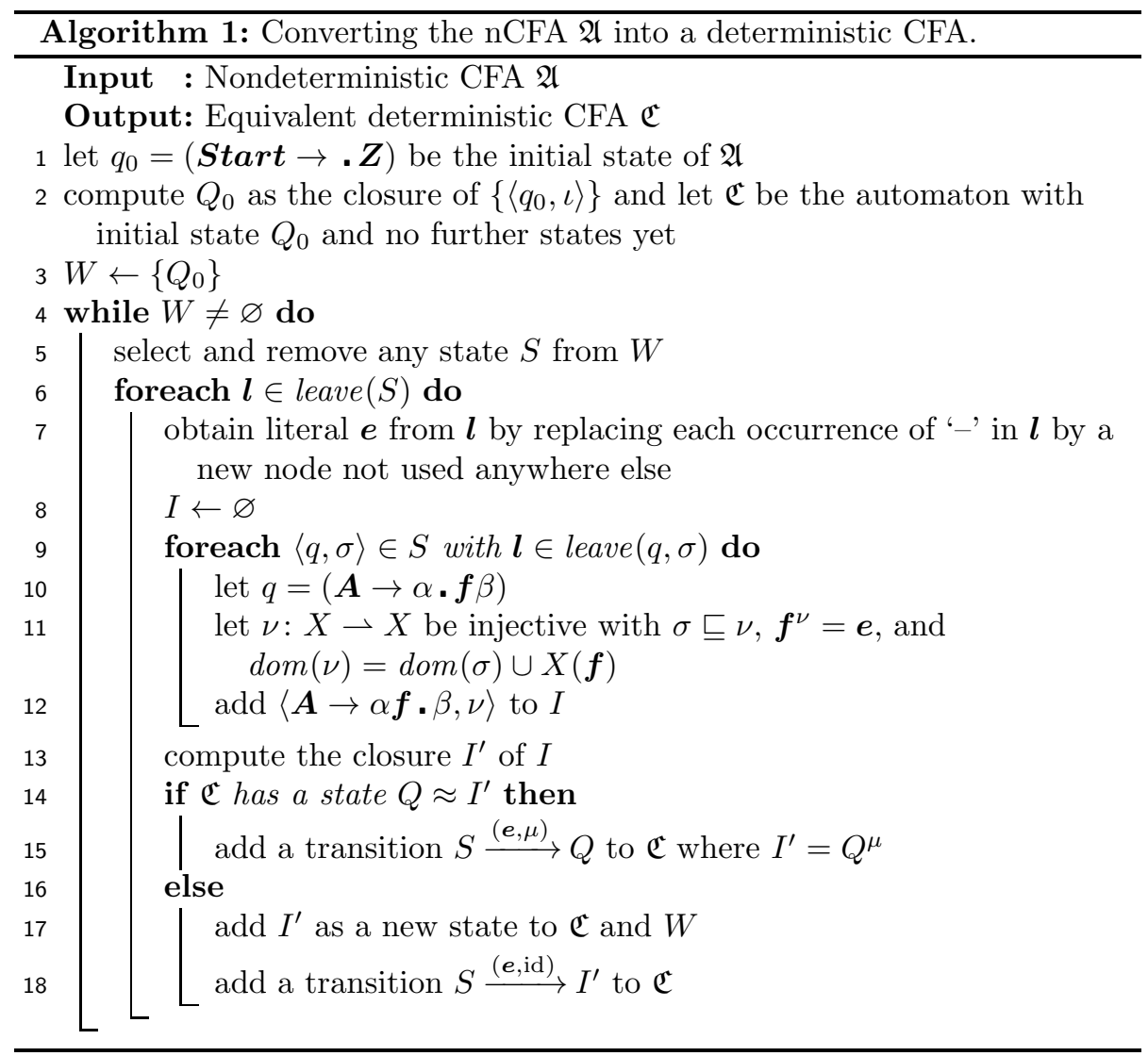

Parameter names can be chosen arbitrarily as long as the parameter mappings are injective. That way, Alg. 1 frequently creates new sets of items which should become states of the dCFA $\mathfrak{C}$, but are equivalent to sets that have already been added as states to $\mathfrak{C}$ and should thus not be added again. Alg. 1 avoids equivalent states (line 14) and "reuses" existing ones instead (line 15). The node mapping being part of transition labels is the parameter renaming that must be applied to reuse an already existing state.

Example 7.5 (The dCFA for the Tree-Generating Grammar). Fig. 10 shows the dCFA obtained by Alg. 1 from the nondeterministic CFA (Fig. 8) for the tree-generating grammar in Example 4.2. It consists of the states $Q_{0}, \ldots, Q_{4}, Q_{\mathrm{A}}$, which are sets of items. Each item $\langle q, \sigma\rangle$ is written in a single line that shows its nCFA state $q$ on the left and its parameter mapping $\sigma$ on the right (in brackets). Each pair $u / v$ denotes that node $u$ of the item is mapped to the parameter $v$, i.e., $\sigma(u)=v ; \sigma$ is undefined for all other nodes. Transitions are labeled by pairs of literals and node mappings. The latter are represented analogously. 


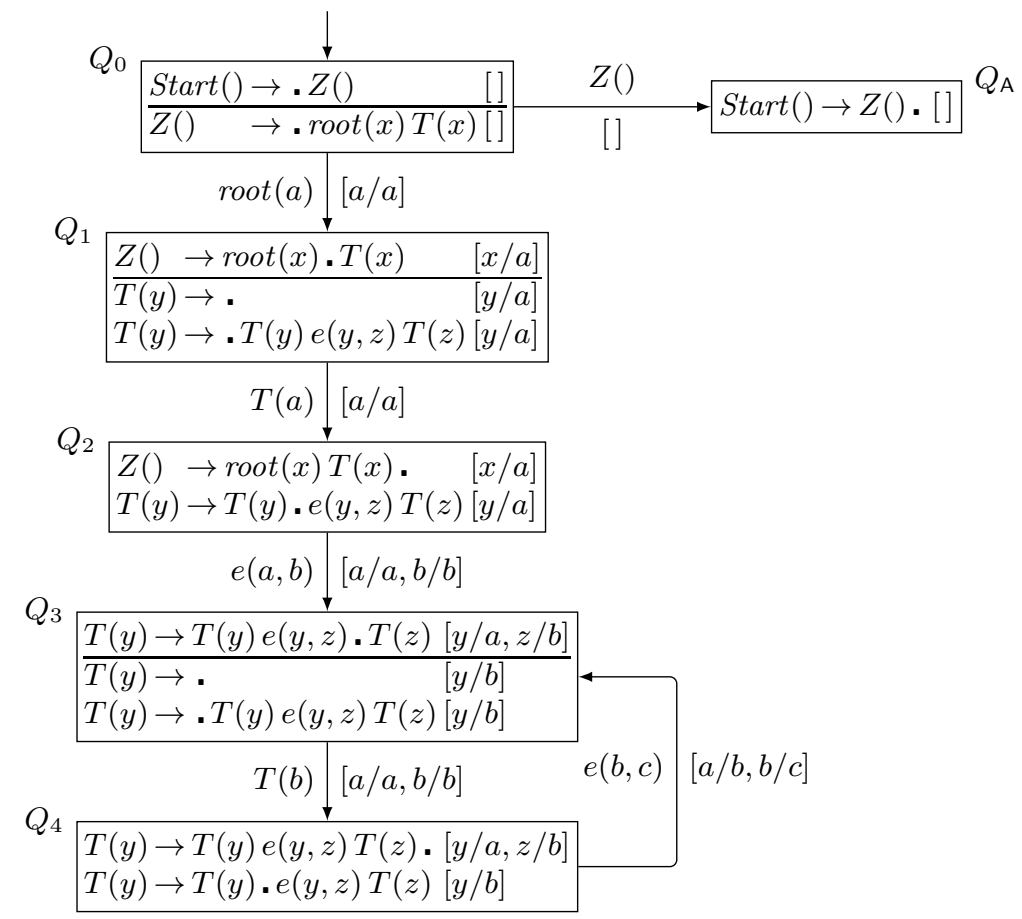

Figure 10: Deterministic CFA created by Alg. 1 from the nCFA in Fig. 8

Note that the three transitions leading from $Q_{0}$ via $Q_{1}$ and $Q_{2}$ to $Q_{3}$ have identities as node mappings because the target states of these transitions were new states when Alg. 1 created the transition (line 18). When it created the transition leaving $Q_{4}$, however, it constructed the set

$$
\begin{aligned}
I^{\prime}=\{\langle T(y) & \rightarrow T(y) e(y, z) \cdot T(z),[y / b, z / c]\rangle, \\
\langle T(y) & \rightarrow \cdot,[y / c]\rangle, \\
\langle T(y) & \rightarrow \cdot T(y) e(y, z) T(z),[y / c]\rangle\}
\end{aligned}
$$

of items, for the new parameter $c$ created in line 7 . But this set is equal to $Q_{3}^{\mu}$ where $\mu=[a / b, b / c]$ is the corresponding node mapping, and Alg. 1 has reused state $Q_{3}$ when adding the transition in line 15 .

Assumption 7.6. Since Alg. 1 constructs the dCFA from an nCFA, and as we have assumed a fixed HR grammar $\Gamma$ and a fixed $n C F A$, we also assume a fixed $\mathrm{dCFA} \mathfrak{C}$ in the following.

The dCFA approves graphs in a similar way as the nCFA does. However, we have to deal with input bindings $\tau$ that map parameters to nodes of the input graph $g$. Formally, an input binding for a dCFA state $Q$ is a total injective function $\tau: \operatorname{params}(Q) \rightarrow X(g)$, where $g$ is the input graph. Here, $g$ may not 


$$
\begin{array}{rlr}
\varepsilon \downarrow Q_{0} & \approx & \operatorname{root}(1) \bullet Q_{1}^{a / 1} \\
1 & \approx & \operatorname{root}(1) T(1) \bullet Q_{2}^{a / 1} \\
1 \approx & \operatorname{root}(1) T(1) e(1,2) \bullet Q_{3}^{a / 1, b / 2} \\
1 \approx & \operatorname{root}(1) T(1) e(1,2) T(2) \bullet Q_{4}^{a / 1, b / 2} \\
1 \approx & \operatorname{root}(1) T(1) e(1,2) T(2) e(2,4) \bullet Q_{3}^{a / 1, b / 2} \\
1 & \approx & \operatorname{root}(1) T(1) e(1,2) T(2) e(2,4) T(4) \bullet Q_{4}^{a / 2, b / 4}
\end{array}
$$

Figure 11: Moves of the dCFA in Fig. 10. Input bindings $\tau$ of the states $Q_{i}^{\tau}$ with $\tau\left(a_{1}\right)=$ $y_{1}, \ldots, \tau\left(a_{k}\right)=y_{k}$ are represented by exponents $a_{1} / y_{1}, \ldots, a_{k} / y_{k}$.

explicitly be given, but is implicitly present as the graph on which the dCFA is run. The item set $Q^{\tau}$ is then a concrete state, which we simply call a state. In other words, a state is a finite set of items in which the parameters are mapped to (pairwise distinct) nodes of the input graph.

We define dCFA configurations, dCFA moves, and approval by a dCFA analogously to their nCFA counterparts (Definitions 6.3, 6.4, and 6.5). The primary difference is that dCFA moves do not have to take closure moves into account:

Definition 7.7. A $d C F A$ configuration $\varphi Q^{\tau}$ consists of a graph $\varphi \in \mathcal{G}_{\Sigma}$ and a state $Q^{\tau}$.

A dCFA transition $t r=\left(Q \stackrel{(\boldsymbol{e}, \mu)}{\longrightarrow} Q^{\prime}\right)$ turns $\varphi Q^{\tau}$ into $\varphi \boldsymbol{e}^{\nu} \bullet Q^{\prime \nu \circ \mu}$ with $\tau \sqsubseteq \nu$, $\operatorname{dom}(\nu)=\operatorname{dom}(\tau) \cup X(\boldsymbol{e})$, and $X\left(\boldsymbol{e}^{\nu}\right) \cap X(\varphi) \subseteq X\left(\boldsymbol{e}^{\tau}\right)$. We write such a $d C F A$ move as $\varphi Q^{\tau} \underset{\text { Ir }}{\approx} \varphi \boldsymbol{e}^{\nu} Q^{\prime \nu \circ \mu}$, where the subscript $\operatorname{tr}$ may be omitted.

The dCFA approves a graph $\varphi \in \mathcal{G}_{\Sigma}$ if there is a dCFA configuration $C=$ $\varphi Q^{\tau}$ such that $\varepsilon Q_{0}^{\iota} \mid \approx{ }^{*} C$.

Example 7.8 (Moves of the dCFA for the Tree-Generating Grammar). Fig. 11 shows moves of the deterministic CFA in Fig. 10. They approve the same graph as the moves (shown in Fig. 9) of the nondeterministic CFA of Fig. 8. Note that the states $Q_{i}^{\tau}$ in Fig. 11 are in fact concrete states.

To prove that the dCFA approves the same graphs as the $\mathrm{nCFA}$, we need the following lemma. It shows that constructing closure items is tightly related to performing closure moves.

Lemma 7.9. $\langle p, \sigma\rangle \triangleright^{*}\langle q, \tau\rangle$ implies $\varphi \diamond[p]^{\mu \circ \sigma}{ }_{{ }_{\mathrm{cl}}} \widetilde{c}^{*} \varphi \diamond[q]^{\mu \circ \tau}$ for all items $\langle p, \sigma\rangle$ and $\langle q, \tau\rangle$, every injective partial function $\mu: X \rightarrow X$ with $\operatorname{dom}(\mu \circ \sigma)=\operatorname{dom}(\sigma)$, and every graph $\varphi \in \mathcal{G}_{\Sigma}$ such that $\varphi \diamond[p]^{\mu \circ \sigma}$ is a valid nCFA configuration.

Proof. Consider any items $\langle p, \sigma\rangle$ and $\langle q, \tau\rangle$ such that $\langle p, \sigma\rangle \triangleright^{n}\langle q, \tau\rangle$, and $\mu$ as well as $\varphi$ as in the lemma. We prove $\operatorname{dom}(\mu \circ \tau)=\operatorname{dom}(\tau)$ and $\varphi \diamond[p]^{\mu \circ \sigma}{ }_{\text {I }{ }_{\mathrm{cl}}}{ }^{n} \varphi \diamond[q]^{\mu \circ \tau}$ by induction on $n$. 
For $n=0,\langle p, \sigma\rangle=\langle q, \tau\rangle$, and therefore, $\varphi \diamond[p]^{\mu \circ \sigma}=\varphi \diamond[q]^{\mu \circ \tau}$ as well as $\operatorname{dom}(\mu \circ \tau)=\operatorname{dom}(\tau)$, as claimed.

For $n>0$, we have $\langle p, \sigma\rangle \triangleright^{n-1}\left\langle p^{\prime}, \sigma^{\prime}\right\rangle \triangleright\langle q, \tau\rangle$, and by the induction hypothesis, $\varphi \diamond[p]^{\mu \circ \sigma}{ }_{\text {I } \widetilde{c l}}^{n-1} \varphi \diamond\left[p^{\prime}\right]^{\mu \circ \sigma^{\prime}}$ and $\operatorname{dom}\left(\mu \circ \sigma^{\prime}\right)=\operatorname{dom}\left(\sigma^{\prime}\right)$. Then $p^{\prime}$ and $q$ are of the form $p^{\prime}=(\boldsymbol{A} \rightarrow \alpha \cdot \boldsymbol{B} \beta), q=(\boldsymbol{C} \rightarrow . \delta), \boldsymbol{C}^{\tau}=\boldsymbol{B}^{\sigma^{\prime}}, \operatorname{dom}(\tau) \subseteq X(\boldsymbol{C})$.

We first show that $\operatorname{dom}(\mu \circ \tau)=\operatorname{dom}(\tau)$. The inclusion $\operatorname{dom}(\mu \circ \tau) \subseteq \operatorname{dom}(\tau)$ follows immediately from the definition of the composition of partial functions. In order to show the opposite inclusion, consider any node $x \in \operatorname{dom}(\tau) \subseteq X(\boldsymbol{C})$. There must be a node $y \in X(\boldsymbol{B})$ such that $\tau(x)=\sigma^{\prime}(y)$ because $\boldsymbol{C}^{\tau}=\overline{\boldsymbol{B}}^{\sigma^{\prime}}$, and therefore $y \in \operatorname{dom}\left(\sigma^{\prime}\right)=\operatorname{dom}\left(\mu \circ \sigma^{\prime}\right)$. In other words, $\tau(x)=\sigma^{\prime}(y) \in \operatorname{dom}(\mu)$, i.e., $x \in \operatorname{dom}(\mu \circ \tau)$, which proves $\operatorname{dom}(\tau) \subseteq \operatorname{dom}(\mu \circ \tau)$.

As a consequence, the equalities $\boldsymbol{C}^{\mu \circ \tau}=\left(\boldsymbol{C}^{\tau}\right)^{\mu}=\left(\boldsymbol{B}^{\sigma^{\prime}}\right)^{\mu}=\boldsymbol{B}^{\mu \circ \sigma^{\prime}}$ and $\operatorname{dom}(\mu \circ \tau)=\operatorname{dom}(\tau) \subseteq X(\boldsymbol{C})$ hold. As $\varphi \diamond\left[p^{\prime}\right]^{\mu \circ \sigma^{\prime}}$ is a valid nCFA configuration, and by Def. 6.4, $\varphi \diamond\left[p^{\prime}\right]^{\mu \circ \sigma^{\prime}}{ }_{\text {Icl }} \varphi \diamond[q]^{\mu \circ \tau}$.

The following Lemma 7.10 shows that each graph approved by the $\mathrm{nCFA}$ is also approved by the dCFA, and Lemma 7.11 shows the opposite direction. But these lemmata are even more specific: by picking the right item of each state from an approving sequence of dCFA states, an approving sequence of nCFA states can be found "within" the sequence of dCFA states. In other words, the relation between the two automata is similar to that between an ordinary nondeterministic finite automaton and its powerset automaton.

Lemma 7.10. For each sequence

$$
\varepsilon \diamond\left[q_{0}\right]^{\iota} \mid \sim * \varphi \diamond[q]^{\varrho}
$$

of $n C F A$ moves, there is a state $Q^{\tau}$ such that $\langle q, \varrho\rangle \in Q^{\tau}$ and

$$
\varepsilon Q_{0}^{\iota} \mid \approx{ }^{*} \varphi Q^{\tau}
$$

Proof. We prove the lemma by induction on the number $n$ of moves in $\varepsilon \diamond\left[q_{0}\right]^{\iota} \mid \sim^{n} \varphi \diamond[q]^{\varrho}$. For $n=0$, it follows immediately from the definition of initial nCFA configurations and $Q_{0}$.

For $n>0$ and the last move being a closure move, the considered sequence of moves is of the form

$$
\varepsilon \diamond\left[q_{0}\right]^{\iota} \sim^{n-1} \varphi \diamond[\boldsymbol{A} \rightarrow \alpha \cdot \boldsymbol{B} \beta]^{\kappa}{ }_{\mathrm{I}}^{\sim} \sim \boldsymbol{C l} \varphi[\boldsymbol{C} \rightarrow . \delta]^{\varrho}
$$

with $\boldsymbol{B}^{\kappa}=\boldsymbol{C}^{\varrho}$ and $\operatorname{dom}(\varrho) \subseteq X(\boldsymbol{C})$. By the induction hypothesis, there is a state $Q^{\tau}$ such that $\varepsilon Q_{0}^{\iota} \mid \approx^{*} \varphi Q^{\tau}$ and $\langle\boldsymbol{A} \rightarrow \alpha \cdot \boldsymbol{B} \beta, \kappa\rangle \in Q^{\tau}$. Therefore, there is an injective $\eta: X \rightarrow X$ with $\kappa=\tau \circ \eta$ and $\langle\boldsymbol{A} \rightarrow \alpha \cdot \boldsymbol{B} \beta, \eta\rangle \in Q$. Since each dCFA state is closed (lines 2 and 13), we also have $\langle\boldsymbol{C} \rightarrow . \delta, \xi\rangle \in Q$ with $\boldsymbol{B}^{\eta}=\boldsymbol{C}^{\xi}$ and $\operatorname{dom}(\xi) \subseteq X(\boldsymbol{C})$. Therefore, $\boldsymbol{C}^{\varrho}=\boldsymbol{B}^{\kappa}=\left(\boldsymbol{B}^{\eta}\right)^{\tau}=\left(\boldsymbol{C}^{\xi}\right)^{\tau}$. And because of injectivity, $\varrho=\tau \circ \xi$, and therefore $\langle\boldsymbol{C} \rightarrow . \delta, \varrho\rangle \in Q^{\tau}$. 
For $n>0$ and the last move being a goto move, the considered sequence of moves is of the form

$$
\varepsilon \diamond\left[q_{0}\right]^{\iota} \sim^{n-1} \varphi \diamond[\boldsymbol{A} \rightarrow \alpha \cdot \boldsymbol{f} \beta]^{\kappa}{ }_{\text {। }{ }_{\text {go }}} \varphi \boldsymbol{f}^{\varrho} \diamond[\boldsymbol{A} \rightarrow \alpha \boldsymbol{f} \cdot \beta]^{\varrho}
$$

with

$$
\kappa \sqsubseteq \varrho, \operatorname{dom}(\varrho)=\operatorname{dom}(\kappa) \cup X(\boldsymbol{f}), \text { and } X\left(\boldsymbol{f}^{\varrho}\right) \cap X(\varphi) \subseteq X\left(\boldsymbol{f}^{\kappa}\right)
$$

By the induction hypothesis, there is a dCFA state $S$ and an input binding $\chi$ such that $\varepsilon Q_{0}^{\iota} \mid \approx{ }^{*} \varphi S^{\chi}$ and $\langle\boldsymbol{A} \rightarrow \alpha \cdot \boldsymbol{f} \beta, \kappa\rangle \in S^{\chi}$. Therefore, there is an injective $\sigma: X \rightarrow X$ with $\kappa=\chi \circ \sigma,\langle\boldsymbol{A} \rightarrow \alpha \cdot \boldsymbol{f} \beta, \sigma\rangle \in S$, and $\boldsymbol{f}^{\sigma} \in$ leave $(S)$. (Note that the identifiers used here match those in Alg. 1.) Alg. 1, therefore, obtained a literal $\boldsymbol{e}$ from $\boldsymbol{f}^{\sigma}$ by replacing each occurrence of '-' by a new node not used anywhere else (line 11). It has also obtained an injective partial function $\nu$ with

$$
\sigma \sqsubseteq \nu, \boldsymbol{f}^{\nu}=\boldsymbol{e} \text {, and } \operatorname{dom}(\nu)=\operatorname{dom}(\sigma) \cup X(\boldsymbol{f})
$$

Alg. 1 added a transition $S \stackrel{(\boldsymbol{e}, \mu)}{\longrightarrow} Q$ to $\mathfrak{C}$ (line 15 or 18) by constructing a set $I^{\prime}$ of items such that $\langle\boldsymbol{A} \rightarrow \alpha \boldsymbol{f} \cdot \beta, \nu\rangle \in I^{\prime}$ (line 12 and 13) and $Q^{\mu}=I^{\prime}$. By the construction of $\boldsymbol{e}$, by (7.1) as well as (7.2), and because $\mu$ is injective, there is an injective $\xi: X \rightarrow X$ with $\chi \sqsubseteq \xi$ and $\varrho=\xi \circ \nu$, and therefore $\langle\boldsymbol{A} \rightarrow \alpha \boldsymbol{f} . \beta, \varrho\rangle \in$ $I^{\prime \xi}=Q^{\xi \circ \mu}$. Since $\boldsymbol{f}^{\varrho}=\boldsymbol{e}^{\xi}$, we can conclude $\varphi S^{\chi} \mid \approx \varphi \boldsymbol{f}^{\varrho} Q^{\xi \circ \mu}$. Thus, the lemma holds with $\tau=\xi \circ \mu$.

Lemma 7.11. For each sequence

$$
\varepsilon Q_{0}^{\iota} \mid{ }^{*} \varphi Q^{\tau}
$$

and each item $\langle q, \vartheta\rangle \in Q^{\tau}$, there exists a sequence

$$
\varepsilon \diamond\left[q_{0}\right]^{\iota} \mid \sim * \varphi \diamond[q]^{\vartheta}
$$

of nCFA moves.

Proof. Let $\varepsilon Q_{0}^{\iota} \mid \approx{ }^{n} \varphi Q^{\tau}$ be any sequence of dCFA moves and $\langle q, \vartheta\rangle$ any item with $\langle q, \vartheta\rangle \in Q^{\tau}$. We prove $\varepsilon \diamond\left[q_{0}\right]^{\iota} \mid \sim \sim^{*} \varphi \diamond[q]^{\vartheta}$ by induction on $n$.

For $n=0$, the proposition follows from $Q=Q_{0}, \varphi=\varepsilon, \tau=\iota$, and therefore $Q^{\tau}=\left\{\left\langle q_{0}, \iota\right\rangle\right\}$, i.e., $q=q_{0}$ and $\vartheta=\iota$.

For $n>0$, there is a sequence of moves

$$
\varepsilon Q_{0}^{\iota}\left|\approx{ }^{n-1} \varphi S^{\chi}\right| \approx \varphi \boldsymbol{e}^{\xi} \cdot Q^{\xi \circ \mu}
$$

with $\tau=\xi \circ \mu, \chi \sqsubseteq \xi, \operatorname{dom}(\xi)=\operatorname{dom}(\chi) \cup X(\boldsymbol{e})$ and the last move using transition $S \stackrel{(\boldsymbol{e}, \mu)}{\longrightarrow} Q$. Alg. 1 added this transition to $\mathfrak{C}$ after computing a set $Q^{\mu}$. (Again, the identifiers used here match those in Alg. 1.) As $\langle q, \vartheta\rangle \in Q^{\xi \circ \mu}$, there is a $\pi$ with $\vartheta=\xi \circ \pi$ and $\langle q, \pi\rangle \in Q^{\mu}=I^{\prime}$. Since $I^{\prime}$ was computed as the 
closure of $I$ (line 13), and by Fact 7.3, there is a item $\langle\boldsymbol{A} \rightarrow \alpha \boldsymbol{f} \cdot \beta, \nu\rangle \in I$ that was added to $I$ in line 12 , and

$$
\langle\boldsymbol{A} \rightarrow \alpha \boldsymbol{f} \cdot \beta, \nu\rangle \triangleright^{*}\langle q, \pi\rangle .
$$

In fact, $\langle\boldsymbol{A} \rightarrow \alpha \boldsymbol{f} \cdot \beta, \nu\rangle$ was added to $I$ after choosing an item $\langle\boldsymbol{A} \rightarrow \alpha \cdot \boldsymbol{f} \beta, \sigma\rangle \in$ $S$, which was turned into $\langle\boldsymbol{A} \rightarrow \alpha \boldsymbol{f} \cdot \beta, \nu\rangle$. Literal $\boldsymbol{e}$ was obtained from $\boldsymbol{f}^{\sigma}$ by replacing each occurrence of '-' by a new node not used anywhere else, and the injective partial function $\nu: X \rightarrow X$ was chosen such that $\sigma \sqsubseteq \nu, \boldsymbol{f}^{\nu}=\boldsymbol{e}$, and $\operatorname{dom}(\nu)=\operatorname{dom}(\sigma) \cup X(\boldsymbol{f})$. By the induction hypothesis, $\varepsilon \diamond\left[q_{0}\right]^{\alpha} \mid \sim^{*} \varphi \diamond[q]^{\kappa}$ for each item $\langle q, \kappa\rangle \in S^{\chi}$, and in particular for $\langle\boldsymbol{A} \rightarrow \alpha \cdot \boldsymbol{f} \beta, \kappa\rangle \in S^{\chi}$ with $\kappa=\chi \circ \sigma$. Let us define $\varrho=\xi \circ \nu$, and therefore $\langle\boldsymbol{A} \rightarrow \alpha \boldsymbol{f} \cdot \beta, \varrho\rangle \in I^{\xi} \subseteq I^{\prime \xi}=Q^{\xi \circ \mu}$ and $\boldsymbol{f}^{\varrho}=\boldsymbol{e}^{\xi}$. By this construction, $\kappa \sqsubseteq \varrho, \operatorname{dom}(\varrho)=\operatorname{dom}(\kappa) \cup X(\boldsymbol{f})$, and $X\left(\boldsymbol{f}^{\varrho}\right) \cap X(\varphi) \subseteq X\left(\boldsymbol{f}^{\kappa}\right)$, and therefore

$$
\varphi \diamond[\boldsymbol{A} \rightarrow \alpha \cdot \boldsymbol{f} \beta]^{\kappa}{ }_{\widetilde{\mathrm{go}}} \varphi \boldsymbol{f}^{\varrho} \diamond[\boldsymbol{A} \rightarrow \alpha \boldsymbol{f} \cdot \beta]^{\varrho}=\varphi \boldsymbol{e}^{\xi} \diamond[\boldsymbol{A} \rightarrow \alpha \boldsymbol{f} \cdot \beta]^{\xi \circ \nu} .
$$

By the construction of $\xi$ and $\nu$, we have $\operatorname{dom}(\xi \circ \nu)=\operatorname{dom}(\nu)$. And, by (7.3) and Lemma 7.9,

$$
\varphi \boldsymbol{e}^{\xi} \diamond[\boldsymbol{A} \rightarrow \alpha \boldsymbol{f} \cdot \beta]^{\xi \circ \nu}{ }^{\mid} \widetilde{c l}^{*} \varphi \boldsymbol{e}^{\xi} \diamond[q]^{\xi \circ \pi}=\varphi \boldsymbol{e}^{\xi} \diamond[q]^{\vartheta},
$$

which completes the proof of the lemma.

An immediate consequence of these lemmata is the following:

Theorem 7.12. A graph is approved by the $d C F A$ if and only if it is approved by the $n C F A$ if and only if it is a viable prefix.

Thm. 7.12 implies that the naïve parser can reach the accepting configuration with some appropriate remaining input if and only if the dCFA approves its current stack.

Before we describe assisted shift-reduce parsers using the deterministic CFA (Sect. 8), let us observe that Alg. 1 may fail to terminate for some HR grammars. An HR grammar for the visual language of structured flowcharts is used to demonstrate this.

Example 7.13 (Structured Flowcharts). Structured flowcharts consist of rectangles containing actions, diamonds that indicate conditions, and ovals indicating begin and end of the program. Arrows indicate control flow; see Fig. 12 for an example. Such flowcharts can be represented by graphs using terminal symbols begin, end, act, and pred where binary act edges represent actions (rectangles) and ternary pred edges conditions (diamonds). Nodes correspond to arrows where edges are attached to the same node if the corresponding components (rectangle, diamond, or oval) are connected by an arrow. The example flowchart in Fig. 12 can be represented by the graph

$$
\operatorname{begin}(a) \operatorname{act}(a, b) \operatorname{pred}(b, c, d) \operatorname{pred}(c, e, f) \operatorname{act}(e, b) \operatorname{act}(f, b) \operatorname{end}(d),
$$




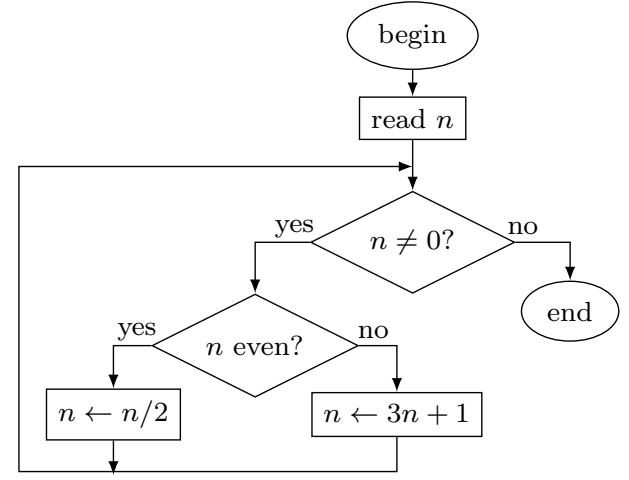

Figure 12: A structured flowchart.

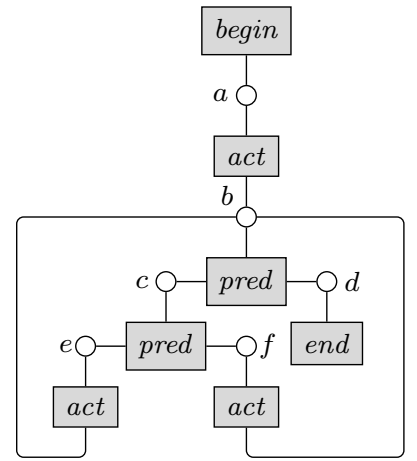

Figure 13: Graph representation of the structured flowchart in Fig. 12.

which can be drawn as the diagram shown in Fig. 13. For instance, literal act $(a, b)$ represents the rectangle "read $n$ ", act $(f, b)$ the rectangle " $n \leftarrow 3 n+$ 1 ", and $\operatorname{pred}(c, e, f)$ the diamond " $n$ even?". An HR grammar for the graph representation of structured flowcharts has four nonterminal symbols Start, Z, Seq (for "sequence"), Stmt (for "statement") and the following rules:

$$
\begin{aligned}
\operatorname{Start}() & \rightarrow Z() & & \\
Z() & \rightarrow \operatorname{begin}(x) \operatorname{Seq}(x, y) \operatorname{end}(y) & & \\
\operatorname{Seq}(x, y) & \rightarrow \operatorname{Stmt}(x, z) \operatorname{Seq}(z, y) & & \text { (sequence) } \\
\operatorname{Seq}(x, y) & \rightarrow \operatorname{Stmt}(x, y) & & \text { (end of sequence) } \\
\operatorname{Stmt}(x, y) & \rightarrow \operatorname{act}(x, y) & & \text { (single action) } \\
\operatorname{Stmt}(x, y) & \rightarrow \operatorname{pred}(x, z, y) \operatorname{Seq}(z, x) & & \text { (while loop) } \\
\operatorname{Stmt}(x, y) & \rightarrow \operatorname{pred}(x, u, v) \operatorname{Seq}(u, y) \operatorname{Seq}(v, y) & & \text { (selection) }
\end{aligned}
$$

The dCFA of this grammar is infinite, i.e., Alg. 1 does not terminate. To see this, consider the excerpt of the dCFA in Fig. 14. To save space, we use a compact notation. If a dCFA state contains items $\left\langle q, \sigma_{1}\right\rangle, \ldots,\left\langle q, \sigma_{n}\right\rangle$ that share the nCFA state $q$, we write $q\left\{\sigma_{1}, \ldots, \sigma_{n}\right\}$ where the parameter mappings $\sigma_{1}, \ldots, \sigma_{n}$ are denoted as introduced earlier. And, if a dCFA state contains the full Cartesian product of a set $Q$ of nCFA states and a set $P$ of parameter mappings, we write $Q \times P$. This notation even allows to represent $Q_{4}$ with its 24 items.

Fig. 14 shows the states $Q_{0}, \ldots, Q_{4}$ of the dCFA and only the transitions between them. One can see that $Q_{2}, Q_{3}$, and $Q_{4}$ are identical when ignoring the parameter mappings. Moreover, when renaming parameters, $Q_{2}$ is properly contained in $Q_{3}$, which in turn is properly contained in $Q_{4}$. These three states are the first states of an infinite sequence $Q_{2}, Q_{3}, Q_{4}, \ldots$, which makes the entire dCFA infinite.

As a consequence, Alg. 1 may fail to terminate. However, a modified algorithm not described here can recognize and handle this situation. For this, it represents the infinite dCFA in a finite way by equipping states with variables 


$$
\begin{aligned}
& \left.Q_{0} \begin{array}{l}
\operatorname{Start}() \rightarrow \cdot Z() \\
Z() \rightarrow \cdot \operatorname{begin}(x) \operatorname{Seq}(x, y) \operatorname{end}(y)\{[]\}
\end{array}\right\} \\
& Q_{1} \quad \operatorname{begin}\left(a_{0}\right) \downarrow\left[a_{0} / a_{0}\right] \\
& \left\{\begin{array}{l}
Z() \rightarrow \operatorname{begin}(x) \cdot \operatorname{Seq}(x, y) \operatorname{end}(y) \\
\operatorname{Seq}(x, y) \rightarrow \cdot \operatorname{Stmt}(x, y) \\
\operatorname{Seq}(x, y) \rightarrow \cdot \operatorname{Stmt}(x, z) \operatorname{Seq}(z, y) \\
\operatorname{Stmt}(x, y) \rightarrow \cdot \operatorname{act}(x, y) \\
\operatorname{Stmt}(x, y) \rightarrow \cdot \operatorname{pred}(x, z, y) \operatorname{Seq}(z, x) \\
\operatorname{Stmt}(x, y) \rightarrow \cdot \operatorname{pred}(x, u, v) \operatorname{Seq}(u, y) \operatorname{Seq}(v, y)
\end{array}\right\} \times\left\{\left[x / a_{0}\right]\right\} \\
& Q_{2} \quad \operatorname{pred}\left(a_{0}, a_{1}, b_{1}\right)\left\lceil\left[a_{0} / a_{0}, a_{1} / a_{1}, b_{1} / b_{1}\right]\right. \\
& \begin{array}{l}
\operatorname{Stmt}(x, y) \rightarrow \operatorname{pred}(x, z, y) \cdot \operatorname{Seq}(z, x) \\
\operatorname{Stmt}(x, y) \rightarrow \operatorname{pred}(x, u, v) \cdot \operatorname{Seq}(u, y) \operatorname{Seq}(v, y) \\
\left\{\begin{array}{l}
\operatorname{Seq}(x, y) \rightarrow \cdot \operatorname{Stmt}(x, y) \\
\operatorname{Seq}(x, y) \rightarrow \cdot \operatorname{Stmt}(x, z) \operatorname{Seq}(z, y) \\
\operatorname{Stmt}(x, y) \rightarrow \cdot \operatorname{act}(x, y) \\
\operatorname{Stmt}(x, y) \rightarrow \cdot \operatorname{pred}(x, z, y) \operatorname{Seq}(z, x) \\
\operatorname{Stmt}(x, y) \rightarrow \cdot \operatorname{pred}(x, u, v) \operatorname{Seq}(u, y) \operatorname{Seq}(v, y)
\end{array}\right\} \times\left\{\begin{array}{l}
\left.\left[x / a_{1}, v / b_{1}, z / a_{1}\right]\right\} \\
\left.\left[x / a_{1}, y / a_{0}\right]\right\}
\end{array}\right\} \\
\end{array}
\end{aligned}
$$

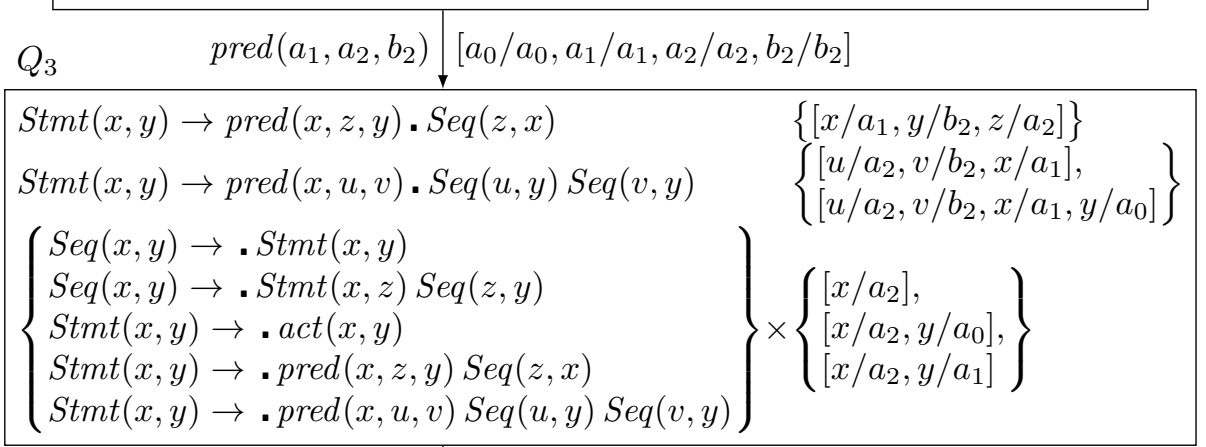

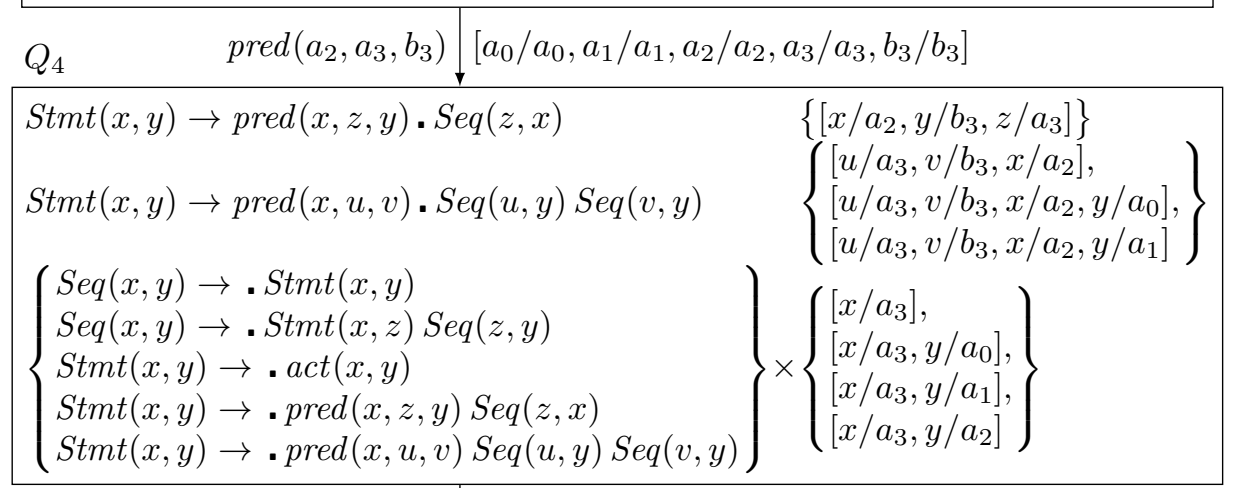

$$
\begin{aligned}
& \operatorname{pred}\left(a_{3}, a_{4}, b_{4}\right) \underset{\ldots}{\downarrow}\left[a_{0} / a_{0}, a_{1} / a_{1}, a_{2} / a_{2}, a_{3} / a_{3}, a_{4} / a_{4}, b_{4} / b_{4}\right]
\end{aligned}
$$

Figure 14: Excerpt of the infinite dCFA of the flowchart grammar. 
that may contain sets of arbitrarily many parameters and using these variables in transitions. This algorithm has been implemented in the Grappa tool. In this paper, we have described the simpler algorithm in Alg. 1 instead of the more general one, because the latter is rather technical. In fact, the HR grammar for structured flowcharts is the only HR grammar with an infinite dCFA known to us - and it is not even PSR parsable because the finitely represented infinite dCFA contains a state with conflicts (see Table 1). We describe the concept of conflicts in Sect. 9.

\section{CFA-Assisted Shift-Reduce Parsing}

Let us now discuss how the naïve shift-reduce parser discussed in Sect. 4 can read off all permissible moves from the current dCFA state in order to reach the accepting configuration with some rest graph, as we shall henceforth call the remaining input. Recall that the naïve parser just maintains a stack of literals. The extended parser, instead, maintains a stack as an alternating sequence of states and literals and makes sure that its stack (when ignoring the states on the stack) is always approved by the dCFA. The top stack element is always the current state, which is the uniquely determined state reached when approving the stack. The stack prior to a move is called current stack, and the next one is the successor stack, thus defining a successor state. The successor stack together with the successor state will then be the current stack and the current state, respectively, at the next move.

When performing a shift move, the parser selects a literal from the remaining input that matches the label of a transition leaving the current state (on top of the stack). This literal is then pushed onto the stack, together with the successor state reachable by this transition. The successor stack thus consists of the current stack, followed by the shifted literal and the successor state.

A reduce move removes the (bound) right-hand side of a rule from the stack, together with the corresponding states, yielding some intermediate stack with a state on top. The parser then selects a transition which leaves the corresponding dCFA state and carries a label matching the reduced nonterminal literal under the same input binding as before. Next, the literal is pushed onto the stack, together with the successor state reachable by this transition. The successor stack thus consists of the intermediate stack, followed by the reduced nonterminal literal and the successor state.

The parser accepts the input graph processed so far when the state on top of the stack is $Q_{\mathrm{A}}=\{\langle\boldsymbol{S t a r t} \rightarrow \boldsymbol{Z} \cdot, \iota\rangle\}$, which is called the accepting state. Note that the entire input graph is accepted that way if there are no unprocessed input literals left when reaching this state, i.e., the rest graph is empty.

Let us now define the extension of the naïve shift-reduce parser more precisely. We call this parser dCFA-assisted shift-reduce parser or simply assisted shift-reduce parser, abbreviated as ASR parser. 
Definition 8.1 (ASR Parser). Let $\mathcal{Q}_{\mathrm{C}}$ denote the set of all (concrete) states. An (ASR parser) configuration $\mathcal{S} \cdot g$ consists of a parse stack $\mathcal{S} \subseteq \mathcal{Q}_{\mathrm{C}} \cdot\left(\right.$ Lit $_{\Sigma} \cdot$ $\left.\mathcal{Q}_{\mathrm{C}}\right)^{*}$ and a graph $g \in \mathcal{G}_{\mathcal{T}}$.

Let $\operatorname{top}(\mathcal{S})$ denote the rightmost element of a parse stack, which is always a state. The graph obtained by removing all states from $\mathcal{S}$ is denoted by $\operatorname{graph}(\mathcal{S})$. A configuration $\mathcal{S} \cdot g$ is accepting if $\operatorname{top}(\mathcal{S})$ is the accepting state $Q_{\mathrm{A}}$ of the dCFA.

An $A S R$ move turns $\mathcal{S} \cdot g$ into $\mathcal{S}^{\prime} \cdot g^{\prime}$ and is either an ASR shift move or an ASR reduce move, defined as follows.

Let $Q^{\tau}=\operatorname{top}(\mathcal{S})$ for a state $Q \in \mathcal{Q}$ and an input binding $\tau: \operatorname{params}(Q) \rightarrow X$.

- Suppose that there is a literal $e \in \operatorname{Lit}_{\mathcal{T}}$ and a state $\bar{Q}$ such that $\operatorname{graph}(\mathcal{S}) \bullet Q^{\tau} \underset{\operatorname{tr}}{\approx} \operatorname{graph}(\mathcal{S}) \boldsymbol{e} \bar{Q}$ and $X(\boldsymbol{e}) \cap X(g) \subseteq X(\operatorname{graph}(\mathcal{S}))$. Then there is an $A S R$ shift move $\mathcal{S} \cdot g \underset{t r}{\models} \mathcal{S e} \bar{Q} \cdot g \boldsymbol{e}$.

- Suppose that $Q$ contains an item it $=\langle\boldsymbol{A} \rightarrow \varrho ., \sigma\rangle$ and one can remove 2 . $|\varrho|$ elements from the top of $\mathcal{S}$ to obtain a parse stack $\mathcal{S}^{\prime \prime}$ with $R=\operatorname{top}\left(\mathcal{S}^{\prime \prime}\right)$ such that there exists a state $\bar{Q}$ with $\operatorname{graph}\left(\mathcal{S}^{\prime \prime}\right) \bullet R \mid \approx \operatorname{graph}\left(\mathcal{S}^{\prime \prime}\right) \boldsymbol{A}^{\tau \circ \sigma} \bullet \bar{Q}$. Then there is an $A S R$ reduce move $\mathcal{S} \cdot g \models_{i t} \mathcal{S}^{\prime \prime} \boldsymbol{A}^{\tau \circ \sigma} \bar{Q} \cdot g$.

We may write $\mathcal{S} \cdot g \models \mathcal{S}^{\prime} \cdot g^{\prime}$ if $\mathcal{S} \cdot g \models_{t r} \mathcal{S}^{\prime} \cdot g^{\prime}$ for a transition $\operatorname{tr}$ or $\mathcal{S} \cdot g \models_{i t} \mathcal{S}^{\prime} \cdot g^{\prime}$ for an item it.

A configuration $\mathcal{S} \cdot g$ can be reached if $Q_{0}^{\iota} \cdot \varepsilon \models^{*} \mathcal{S} \cdot g$. An ASR parser accepts a graph $g \in \mathcal{G}_{\mathcal{T}}$ if it can reach an accepting configuration $\mathcal{S} \cdot g^{\prime}$ for a graph $g^{\prime} \bowtie g$.

Note that shift and reduce moves of the ASR parser always push (concrete) states onto the stack that are reachable from their immediate predecessor states on the stack. This is expressed in the following fact:

Fact 8.2. $Q_{0}^{\iota} \cdot \varepsilon \models^{*} \mathcal{S} \cdot g$ implies $\varepsilon Q_{0}^{\iota} \mid \approx{ }^{*} \operatorname{graph}\left(\mathcal{S}^{\prime}\right) \operatorname{top}\left(\mathcal{S}^{\prime}\right)$ for every $A S R$ parser configuration $\mathcal{S} \cdot g$ and every parse stack $\mathcal{S}^{\prime}$ being a prefix of $\mathcal{S}$.

Example 8.3 (An ASR Parse of a Tree). Fig. 15 shows the moves of the ASR parser when recognizing the tree in Example 4.2. ${ }^{5}$ Moves 1-6 in Fig. 15 correspond to the moves of the dCFA shown in Fig. 11 of Example 7.8 in the way stated in Fact 8.2:

- The initial configuration of the ASR parser agrees with the initial state of the dCFA.

- The literal and state pushed in move $i$ agrees with the literal processed, and the state reached, by move $i$ of the dCFA. In three steps, terminal literals are pushed by shift moves; the other moves push nonterminal literals

\footnotetext{
${ }^{5}$ In this example and in Fig. 15, we use following abbreviated notation: literals $\ell\left(x_{1}, \ldots, x_{k}\right)$ are denoted as $\ell^{x_{1} \ldots x_{k}}$, and (concrete) states of the dCFA that were written as $Q_{i}^{\left[a_{1} / y_{1}, \ldots, a_{k} / y_{k}\right]}$ in Fig. 11 are abbreviated as $Q_{i}^{y_{1} \ldots y_{k}}$.
} 


\begin{tabular}{|c|c|c|}
\hline & stack . read input & match \\
\hline & $Q_{0} \cdot \varepsilon$ & \\
\hline$\models$ & $Q_{0} \operatorname{root}^{1} Q_{1 \_}^{1} \cdot \operatorname{root}^{1}$ & \\
\hline$\models$ & $Q_{0} \operatorname{root}^{1} Q_{1}^{1} T^{1} Q_{2}^{1} \cdot \operatorname{root}^{1}$ & $y / 1$ \\
\hline$\stackrel{3}{=}$ & $Q_{0} \operatorname{root}^{1} Q_{1}^{1} T^{1} Q_{2}^{1} e^{12} Q_{3}^{12}{ }_{-} \operatorname{root}^{1} e^{12}$ & \\
\hline$\models$ & $Q_{0} \operatorname{root}^{1} Q_{1}^{1} T^{1} Q_{2}^{1} e^{12} Q_{3}^{12} T^{2} Q_{4}^{12} \cdot \operatorname{root}^{1} e^{12}$ & $y / 2$ \\
\hline$\stackrel{\varrho}{\models}$ & $Q_{0} \operatorname{root}^{1} Q_{1}^{1} T^{1} Q_{2}^{1} e^{12} Q_{3}^{12} T^{2} Q_{4}^{12} e^{24} Q_{3}^{24} \cdot \operatorname{root}^{1} e^{12} e^{24}$ & $x / 2, y / 4$ \\
\hline$\models$ & $Q_{0} \operatorname{root}^{1} Q_{1}^{1} T^{1} Q_{2}^{1} e^{12} Q_{3}^{12} \underline{T}^{2} Q_{4}^{12} e^{24} Q_{3}^{24} T^{4} Q_{4}^{24} \cdot \operatorname{root}^{1} e^{12} e^{24}$ & $y / 4$ \\
\hline$\models$ & $Q_{0} \operatorname{root}^{1} Q_{1}^{1} \underline{T}^{1} Q_{2}^{1} e^{12} Q_{3}^{12} T^{2} Q_{4}^{12} \cdot \operatorname{root}^{1} e^{12} e^{24}$ & $x / 2, y / 4$ \\
\hline$\models$ & $Q_{0} \operatorname{root}^{1} Q_{1}^{1} T^{1} Q_{2}^{1} \cdot \operatorname{root}^{1} e^{12} e^{24}$ & $x / 1, y / 2$ \\
\hline$\stackrel{2}{\models}$ & $Q_{0} \operatorname{root}^{1} Q_{1}^{1} T^{1} Q_{2}^{1} e^{13} Q_{3}^{13}{ }_{-} \cdot \operatorname{root}^{1} e^{12} e^{24} e^{13}$ & \\
\hline$\models_{3}$ & $Q_{0} \operatorname{root}^{1} Q_{1}^{1} \underline{T^{1} Q_{2}^{1} e^{13} Q_{3}^{13} T^{3} Q_{4}^{1 \overline{3}}} \cdot \operatorname{root}^{1} e^{12} e^{24} e^{13}$ & $y / 3$ \\
\hline$\models$ & $Q_{0} \underline{\operatorname{root}^{1} Q_{1}^{1} T^{1} Q_{2}^{1}} \cdot \operatorname{root}^{1} e^{12} e^{24} e^{13}$ & $x / 1, y / 3$ \\
\hline$\models^{2}$ & $Q_{0} Z Q_{\mathrm{A}} \cdot \operatorname{root}^{1} e^{12} e^{24} e^{13}$ & \\
\hline
\end{tabular}

Figure 15: Moves of the ASR parser recognizing the tree in Example 4.2. Places on the stack where reductions occur are underlined. Rules used in reduce moves are indicated as subscripts in the leftmost column, and their corresponding matches appear in the rightmost column.

with label $T$ in the course of reducing rule 2 or rule 3 . The former has a right-hand side of length three; six elements are thus popped off the stack, whereas the latter has no literals on its right-hand side so that nothing has to be popped off the stack.

- After move $i$, the symbols on the stack of the parser (ignoring the states) agree with the viable prefix approved in move $i$ of the dCFA.

Note also that a reduce move of the naïve shift-reduce parser must check a rather complex condition in order to select a reduce move (Def. 4.4); it must examine whether the stack contains the right hand side of the rule (under an appropriate match), and it must additionally check condition $X(\alpha) \cap X\left(\varrho^{\mu}\right) \subseteq$ $X\left(\boldsymbol{A}^{\mu}\right)$ of Def. 4.4 to make sure that the corresponding derivation step is valid. The ASR parser, instead, just inspects the top state on the stack and checks whether this state contains an item with the dot at the end of the rule; it can thus read off from the dCFA whether it can select a reduce move. The following lemma states this formally. It will be used for proving the correctness of the ASR parser later.

Lemma 8.4. For every rule $\boldsymbol{A} \rightarrow \varrho$ with $\boldsymbol{A} \neq$ Start and every sequence $\varepsilon Q_{0}^{\iota} \mid \approx{ }^{*} \varphi Q^{\tau}, Q$ contains an item $\langle\boldsymbol{A} \rightarrow \varrho \cdot, \sigma\rangle$ if and only if there is a graph $\alpha \in \mathcal{G}_{\Sigma}$ such that $\varphi=\alpha \varrho^{\tau \circ \sigma}, \alpha \boldsymbol{A}^{\tau \circ \sigma}$ is also approved by the $d C F A$, and $X(\alpha) \cap X\left(\varrho^{\tau \circ \sigma}\right) \subseteq X\left(\boldsymbol{A}^{\tau \circ \sigma}\right)$. 
Proof. For the only-if direction, consider a sequence $\varepsilon Q_{0}^{\iota} \mid \approx^{*} \varphi Q^{\tau}$ and an item $\langle\boldsymbol{A} \rightarrow \varrho ., \sigma\rangle \in Q$. We have $\langle\boldsymbol{A} \rightarrow \varrho ., \mu\rangle \in Q^{\tau}$ with $\mu=\tau \circ \sigma$, and by Lemma 7.11, there is a sequence

$$
\varepsilon \diamond\left[q_{0}\right]^{L} \sim^{*} \varphi \diamond[\boldsymbol{A} \rightarrow \varrho \cdot]^{\mu} .
$$

The dot in $\boldsymbol{A} \rightarrow \varrho$. must have been moved there by goto moves, starting at $\boldsymbol{A} \rightarrow \boldsymbol{\bullet} \varrho$, an nCFA state that was reached by a closure move. Therefore, (8.1) reads

$$
\varepsilon \diamond\left[q_{0}\right]^{\iota} \sim^{*} \alpha \diamond[\boldsymbol{B} \rightarrow \gamma \cdot \boldsymbol{C} \delta]^{\nu}{ }_{\text {|cl }} \alpha \diamond[\boldsymbol{A} \rightarrow \cdot \varrho]^{\mu^{\prime}}{ }_{{ }_{\mathrm{go}}} * \alpha \varrho^{\mu} \diamond[\boldsymbol{A} \rightarrow \varrho \cdot]^{\mu}
$$

with $\varphi=\alpha \varrho^{\mu}=\alpha \varrho^{\tau \circ \sigma}, \boldsymbol{C}^{\nu}=\boldsymbol{A}^{\mu^{\prime}}, \mu^{\prime} \sqsubseteq \mu, X(\boldsymbol{A}) \subseteq X(\varrho)=\operatorname{dom}(\mu)$. Thus $\boldsymbol{A}^{\mu}$ is a literal, and there is an injective $\nu^{\prime}: X \rightarrow X$ with $\nu \sqsubseteq \nu^{\prime}, \operatorname{dom}\left(\nu^{\prime}\right)=$ $\operatorname{dom}(\nu) \cup X(\boldsymbol{C})$, and $\boldsymbol{C}^{\nu^{\prime}}=\boldsymbol{A}^{\mu}$. As a consequence, there is also a sequence

$$
\varepsilon \diamond\left[q_{0}\right]^{\iota} \sim^{*} \alpha \diamond[\boldsymbol{B} \rightarrow \gamma \cdot \boldsymbol{C} \delta]^{\nu}{ }^{{ }_{\text {go }}} \alpha \boldsymbol{A}^{\mu} \diamond[\boldsymbol{B} \rightarrow \gamma \boldsymbol{C} \cdot \delta]^{\nu^{\prime}}
$$

showing that $\alpha \boldsymbol{A}^{\mu}$ is approved by the nCFA and consequently, using Lemma 7.10, by the dCFA. Moreover, $X(\alpha) \cap X\left(\varrho^{\mu}\right) \subseteq X\left(\varrho^{\mu^{\prime}}\right)=X\left(\boldsymbol{A}^{\mu^{\prime}}\right) \subseteq X\left(\boldsymbol{A}^{\mu}\right)$ using Lemma 6.8 .

For the if direction, let $\boldsymbol{A} \rightarrow \varrho$ be a rule with $\boldsymbol{A} \neq \boldsymbol{S t a r t}$, and consider a sequence $\varepsilon Q_{0}^{\iota} \mid \approx{ }^{*} \alpha \varrho^{\mu} Q^{\tau}$ with an injective $\mu: X \rightarrow X$, and $\varepsilon Q_{0}^{\iota} \mid \approx^{*} \alpha \boldsymbol{A}^{\mu} \widehat{Q}^{\xi}$ for some dCFA state $\widehat{Q}$ and injective $\xi: X \rightarrow X$. By Lemma 7.11,

$$
\varepsilon \diamond\left[q_{0}\right]^{\iota} \sim^{*} \alpha \diamond[\boldsymbol{B} \rightarrow \gamma \cdot \boldsymbol{C} \delta]^{\nu^{\prime}}{ }_{{ }_{\text {go }}} \alpha \boldsymbol{A}^{\mu} \diamond[\boldsymbol{B} \rightarrow \gamma \boldsymbol{C} \cdot \delta]^{\nu}
$$

with $\boldsymbol{A}^{\mu}=\boldsymbol{C}^{\nu}, \nu^{\prime} \sqsubseteq \nu$, and $\operatorname{dom}(\nu)=\operatorname{dom}\left(\nu^{\prime}\right) \cup X(\boldsymbol{C})$. Therefore, we also have

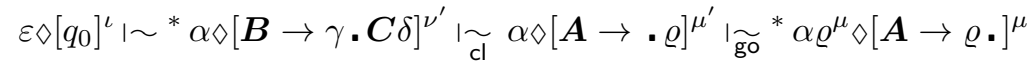

with $\boldsymbol{C}^{\nu^{\prime}}=\boldsymbol{A}^{\mu^{\prime}}$ and, by Lemma 7.10, $\varepsilon Q_{0}^{\iota} \mid \approx{ }^{*} \alpha \varrho^{\mu} \bullet Q^{\prime \tau^{\prime}}$ with a dCFA state $Q^{\prime}$, injective $\tau^{\prime}: X \rightarrow X$, and $\langle\boldsymbol{A} \rightarrow \varrho ., \mu\rangle \in Q^{\prime \tau^{\prime}}$. In fact, $Q=Q^{\prime}$ and $\tau=\tau^{\prime}$ since the dCFA is deterministic. Hence, $Q$ contains an item $\langle\boldsymbol{A} \rightarrow \varrho ., \sigma\rangle$ with $\mu=\tau \circ \sigma$.

We are now ready to prove that the ASR parser is in fact an improved version of the naïve shift-reduce parser (Def. 4.4) that always makes sure that its stack is a viable prefix:

Lemma 8.5. For every $A S R$ parser configuration $\mathcal{S} . g$ with $\operatorname{graph}(\mathcal{S}) \neq$ Start and every $n \in \mathbb{N}, Q_{0}^{\iota} \cdot \varepsilon \models^{n} \mathcal{S} \cdot g$ if and only if $\varepsilon Q_{0}^{\iota} \mid \approx^{*} \varphi R$ and $\varepsilon \cdot \varepsilon \vdash^{n} \varphi \cdot g$ where $R=\operatorname{top}(\mathcal{S})$ and $\varphi=\operatorname{graph}(\mathcal{S})$.

Proof. We prove the lemma by induction on $n$. For $n=0$, it immediately follows from the fact that $\mathcal{S}=\operatorname{top}(\mathcal{S})=Q_{0}^{\iota}$ and $\operatorname{graph}(\mathcal{S})=g=\varepsilon$.

For the inductive step, let $n \geq 0$. We show that the statement holds for $n+1$ under the assumption that it holds for all shorter configuration sequences of length up to $n$. We show the only-if and the if direction separately: 
(1) To show the only-if direction, we assume any sequence

$$
Q_{0}^{\iota} \cdot \varepsilon \models^{n} \mathcal{S}^{\prime} \cdot g^{\prime} \models \mathcal{S} \cdot g .
$$

Let $R=\operatorname{top}\left(\mathcal{S}^{\prime}\right)$ and $\varphi=\operatorname{graph}\left(\mathcal{S}^{\prime}\right)$. The last move is either a shift move or a reduce move.

(1a) If it is a shift move, there exist a literal $e \in$ Lit $_{\mathcal{T}}$ and a state $T$ with

$$
\begin{aligned}
\varphi \bullet R & \approx \varphi \boldsymbol{e} T \\
X(\boldsymbol{e}) \cap X(g) & \subseteq X(\varphi) \\
\mathcal{S} & =\mathcal{S}^{\prime} \boldsymbol{e} T \\
g & =g^{\prime} \boldsymbol{e} .
\end{aligned}
$$

Now, $\varepsilon Q_{0}^{\iota}\left|\approx{ }^{*} \varphi R\right| \approx \varphi \boldsymbol{e} T$ follows from (8.2) and the induction

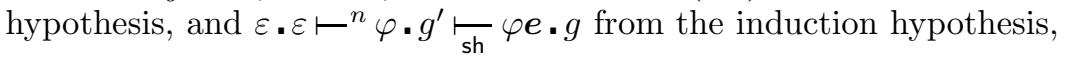
(8.3), (8.5), and Def. 4.4. This concludes case (1a) because $\operatorname{top}\left(\mathcal{S}^{\prime}\right)=$ $T$ and $\operatorname{graph}\left(\mathcal{S}^{\prime}\right)=\varphi \boldsymbol{e}$.

(1b) If the last move is a reduce move, there is a rule $\boldsymbol{A} \rightarrow \varrho$, and one can obtain a parse stack $\mathcal{S}^{\prime \prime}$ by removing $2 \cdot|\varrho|$ elements from the end of $\mathcal{S}^{\prime}$. Let $\psi=\operatorname{graph}\left(\mathcal{S}^{\prime \prime}\right)$ and $Q=\operatorname{top}\left(\mathcal{S}^{\prime \prime}\right)$. By Def. 8.1, there is a state $T$ and a dCFA state $Q_{i}$ containing an item $\langle\boldsymbol{A} \rightarrow \varrho ., \sigma\rangle$ such that

$$
\begin{aligned}
\psi & Q \mid \approx \boldsymbol{A}^{\tau \circ \sigma} \diamond T \\
\mathcal{S} & =\mathcal{S}^{\prime \prime} \boldsymbol{A}^{\tau \circ \sigma} T \\
g & =g^{\prime} .
\end{aligned}
$$

By Lemma 8.4, there is a graph $\alpha \in \mathcal{G}_{\Sigma}$ and a state $T^{\prime}$ such that

$$
\begin{aligned}
\varphi & =\alpha \varrho^{\tau \circ \sigma} \\
\varepsilon \bullet Q_{0}^{\iota} & \approx{ }^{*} \alpha \boldsymbol{A}^{\tau \circ \sigma} T^{\prime} \\
X(\alpha) \cap X\left(\varrho^{\tau \circ \sigma}\right) & \subseteq X\left(\boldsymbol{A}^{\tau \circ \sigma}\right) .
\end{aligned}
$$

Now, $\alpha=\psi$ follows from the construction of $\mathcal{S}^{\prime \prime}$ and

$$
\varepsilon Q_{0}^{\iota} \mid \approx^{*} \alpha \boldsymbol{A}^{\tau \circ \sigma} \bullet T^{\prime}=\psi \boldsymbol{A}^{\tau \circ \sigma} \bullet T
$$

from (8.6), (8.10), and the fact that the dCFA is deterministic. Finally,

$$
\varepsilon \cdot \varepsilon \vdash^{n} \varphi \cdot g^{\prime}=\psi \varrho^{\tau \circ \sigma} \cdot g ঙ_{\boldsymbol{A}^{\tau \circ \sigma} \Rightarrow \varrho^{\tau \circ \sigma}} \psi A^{\tau \circ \sigma} \cdot g
$$

using the induction hypothesis, (8.8), (8.9), (8.11) and Def. 4.4. This concludes case $(1 \mathrm{~b})$ because $\operatorname{top}(\mathcal{S})=T$ and $\operatorname{graph}(\mathcal{S})=\psi A^{\tau \circ \sigma}$.

(2) To show the if direction, we now assume any sequence

$$
\varepsilon \cdot \varepsilon \vdash^{n} \varphi^{\prime} \cdot g^{\prime} \vdash \varphi \cdot g .
$$

of moves and

$$
\varepsilon Q_{0}^{\iota} \mid{ }^{*} \varphi
$$

for a state $R$. The last move in (8.12) is either a shift or a reduce move. 
(2a) If it is a shift move, there exists a literal $e \in \operatorname{Lit}_{\mathcal{T}}$ such that

$$
\begin{aligned}
\varphi & =\varphi^{\prime} \boldsymbol{e} \\
g & =g^{\prime} \boldsymbol{e} \\
X(\boldsymbol{e}) \cap X\left(g^{\prime}\right) & \subseteq X\left(\varphi^{\prime}\right)
\end{aligned}
$$

Because of (8.14), we can write (8.13) as

$$
\varepsilon \triangleleft Q_{0}^{\iota}\left|\approx{ }^{*} \varphi^{\prime} \otimes\right| \approx \varphi^{\prime} e \bullet R
$$

for some state $Q$. Therefore, the induction hypothesis applies and yields $Q_{0}^{\iota} \cdot \varepsilon \models^{n} \mathcal{S}^{\prime} \cdot g^{\prime}$ with $\operatorname{top}\left(\mathcal{S}^{\prime}\right)=Q$ and $\operatorname{graph}\left(\mathcal{S}^{\prime}\right)=\varphi^{\prime}$. Finally, because of (8.15), (8.16) and (8.17), there is a shift move

$$
\mathcal{S}^{\prime} \cdot g^{\prime} \models \mathcal{S}^{\prime} e R \cdot g^{\prime} \boldsymbol{e}=\mathcal{S} \cdot g
$$

with $\mathcal{S}=\mathcal{S}^{\prime} \boldsymbol{e} R$ and, therefore, $\operatorname{top}(\mathcal{S})=R$ and $\operatorname{graph}(\mathcal{S})=\varphi^{\prime} \boldsymbol{e}=\varphi$ because of (8.14), which concludes case (2a).

(2b) If the last move is a reduce move, there is a rule $\boldsymbol{A} \rightarrow \varrho$, a match $\mu: X \rightarrow X$, and a graph $\alpha \in \mathcal{G}_{\Sigma}$ such that

$$
\begin{aligned}
\varphi^{\prime} & =\alpha \varrho^{\mu} \\
\varphi & =\alpha \boldsymbol{A}^{\mu} \\
g & =g^{\prime} \\
X(\alpha) \cap X\left(\varrho^{\mu}\right) & \subseteq X\left(\boldsymbol{A}^{\mu}\right)
\end{aligned}
$$

and (8.13) can be written as

$$
\varepsilon Q_{0}^{\iota} \mid \approx^{*} \alpha \boldsymbol{A}^{\mu} \diamond R
$$

The graph $\varphi=\alpha \boldsymbol{A}^{\mu}$ is a viable prefix because of (8.13), Thm. 6.12, and Thm. 7.12. Therefore, $\varphi^{\prime}=\alpha \varrho^{\mu}$ is also a viable prefix because of $\varphi=\alpha \boldsymbol{A}^{\mu} \Rightarrow \alpha \varrho^{\mu}=\varphi^{\prime}$. Since the grammar is reduced, there must be states $Q, Q^{\prime}$ such that

$$
\varepsilon Q_{0}^{\iota}\left|\approx^{*} \alpha Q^{\prime}\right| \approx{ }^{*} \alpha \varrho^{\mu} \bullet Q=\varphi^{\prime} \bullet Q
$$

Because of (8.12), there is also a sequence $\varepsilon \cdot \varepsilon \vdash^{k} \alpha \cdot g^{\prime \prime}$ for some prefix $g^{\prime \prime}$ of $g=g^{\prime}$ and $k \leq n$. Therefore, the induction hypothesis applies, and we can conclude

$$
Q_{0}^{\iota} \cdot \varepsilon \models^{k} \mathcal{S}^{\prime \prime} \cdot g^{\prime \prime}
$$

for a parse stack $\mathcal{S}^{\prime \prime}$ with $\operatorname{top}\left(\mathcal{S}^{\prime \prime}\right)=Q^{\prime}$ and $\operatorname{graph}\left(\mathcal{S}^{\prime \prime}\right)=\alpha$. Using the same argument, we can also conclude

$$
Q_{0}^{\iota} \cdot \varepsilon \models^{n} \mathcal{S}^{\prime} \cdot g^{\prime}
$$

for a parse stack $\mathcal{S}^{\prime}$ with $\operatorname{top}\left(\mathcal{S}^{\prime}\right)=Q$ and $\operatorname{graph}\left(\mathcal{S}^{\prime}\right)=\alpha \varrho^{\mu}=\varphi^{\prime}$. 
Let us assume that $\mathcal{S}^{\prime \prime}$ is not a prefix of $\mathcal{S}^{\prime}$. There must be a parse stack $\hat{\mathcal{S}}$, literal $\boldsymbol{l}$ and states $P^{\prime}, P^{\prime \prime}, P^{\prime} \neq P^{\prime \prime}$, such that $\hat{\mathcal{S}} \boldsymbol{l} P^{\prime}$ is a prefix of $\mathcal{S}^{\prime}$ and $\hat{\mathcal{S}} \boldsymbol{l} P^{\prime \prime}$ a prefix of $\mathcal{S}^{\prime \prime}$. Let $\psi=\operatorname{graph}\left(\hat{\mathcal{S}} \boldsymbol{l} P^{\prime}\right)=\operatorname{graph}\left(\hat{\mathcal{S}} \boldsymbol{l} P^{\prime \prime}\right)$. We can conclude $\varepsilon Q_{0}^{\iota} \mid \approx{ }^{*} \psi P^{\prime}$ and $\varepsilon Q_{0}^{\iota} \mid \approx^{*} \psi P^{\prime \prime}$ using Fact 8.2, and $P^{\prime}=P^{\prime \prime}$ using the fact that the dCFA is deterministic, contradicting our assumption. $\mathcal{S}^{\prime \prime}$ is thus a prefix of $\mathcal{S}^{\prime}$, and $\mathcal{S}^{\prime \prime}$ can be obtained from $\mathcal{S}^{\prime}$ by removing $2 \cdot|\varrho|$ elements from its end.

Because of $(8.21),(8.22),(8.23)$, and Lemma 8.4, there is a dCFA state $Q_{i}$, an input binding $\tau$ and an item $\langle\boldsymbol{A} \rightarrow \varrho ., \sigma\rangle \in Q_{i}$ such that

$$
\begin{aligned}
& Q=Q_{i}^{\tau} \\
& \mu=\tau \circ \sigma .
\end{aligned}
$$

Moreover, we know that

$$
\alpha Q^{\prime} \mid \approx \alpha A^{\mu} \diamond R
$$

by (8.22) and (8.23), using the fact that the dCFA is deterministic. Therefore, using Def. 8.1,

$$
Q_{0}^{\iota} \cdot \varepsilon \models^{n} \mathcal{S}^{\prime} \cdot g^{\prime} \models \mathcal{S}^{\prime \prime} \boldsymbol{A}^{\mu} R \cdot g^{\prime} .
$$

This concludes case (2b) and with it the proof of the lemma because of (8.20) and (8.25), choosing $\mathcal{S}=\mathcal{S}^{\prime \prime} \boldsymbol{A}^{\tau \circ \sigma} R$.

We are now ready to prove the correctness of the ASR parser.

Theorem 8.6. Let $g \in \mathcal{G}_{\mathcal{T}}$. The ASR parser can reach an accepting configuration $\mathcal{S} . g$ if and only if $\boldsymbol{Z} \Rightarrow^{*} g$. Moreover, for every reachable configuration $\mathcal{S} \cdot g$, there is a graph $g^{\prime} \in \mathcal{G}_{\mathcal{T}}$ and an accepting configuration $\mathcal{S}^{\prime} \cdot g g^{\prime}$ such that $\mathcal{S} \cdot g \models^{*} \mathcal{S}^{\prime} \cdot g g^{\prime}$.

Proof. Consider any graph $g \in \mathcal{G}_{\mathcal{T}}$.

For the first part of the theorem, by Thm. 4.9 it holds that $\boldsymbol{Z} \Rightarrow^{*} g$ if and only if $\varepsilon \cdot \varepsilon \vdash^{*} \boldsymbol{Z} \cdot g$. By Lemma 8.5, the latter is the case if an only if $Q_{0}^{\iota} \cdot \varepsilon \models^{*}$ $Q_{0}^{\iota} \boldsymbol{Z} Q_{\mathrm{A}} \cdot g$, because the dCFA approves the viable prefix $\boldsymbol{Z}$ via $\varepsilon Q_{0} \mid \approx{ }^{*} \boldsymbol{Z} \bullet Q_{\mathrm{A}}$.

To prove the second part of the theorem, consider any configuration $\mathcal{S} \cdot g$ with $Q_{0}^{\iota} \cdot \varepsilon \models^{*} \mathcal{S} \cdot g$. By Lemma 8.5, Thm. 6.12, and Thm. 7.12, $\operatorname{graph}(\mathcal{S})$ is a viable prefix. Moreover, $\varepsilon \cdot \varepsilon \vdash^{*} \operatorname{graph}(\mathcal{S}) \cdot g$. By Lemma 5.5, there is a graph $g^{\prime} \in \mathcal{G}_{\mathcal{T}}$ such that $\operatorname{graph}(\mathcal{S}) \cdot g \vdash^{*} \boldsymbol{Z} \cdot g g^{\prime}$. Thus, the same argument as above yields $\mathcal{S} \cdot g \models^{*} Q_{0}^{\iota} \boldsymbol{Z} Q_{\mathrm{A}} \cdot g g^{\prime}$, and its final configuration is accepting.

It is worthwhile pointing out that the ASR parser is still nondeterministic, despite the "assistance" by the dCFA. In fact, there are two sources of nondeterminism. First, the state on top of the stack may contain several items that fulfill the conditions of shift or reduce moves and thus enable several possible moves. There may be items leading to shifts of different literals, items that result in reductions according to different rules, and items of which one triggers 
a shift move whereas the other triggers a reduce move. For example, in state $Q_{1}$ of the dCFA in Fig. 14 (under some input binding), the parser may choose among three shift moves.

The second source of nondeterminism lies in the choice of the edge to be read by a shift move, as there may be several literals $\boldsymbol{e}$ in the input graph that fulfill the conditions.

Naturally, the "right" choice must be made in order to ensure that the parser accepts a given input graph. Note that this does not contradict Thm. 8.6 which states that, regardless of the choice made, there exists a possible rest graph with which the parser can reach an accepting configuration. Clearly, that rest graph can differ from the actual rest graph in the input. Looking at the ASR parser, this observation should not come as a surprise, because the parser does not inspect the rest graph in any way (except for selecting a literal to be shifted whenever a shift move is made). The extension of the ASR parser by an appropriate inspection of the rest graph to predict the necessary move will be discussed next. It leads to the main notion proposed in this paper, the predictive shift-reduce parser.

\section{Predictive Shift-Reduce Parsing}

Intuitively, a move of the parser is appropriate if it keeps it on its way towards accepting the input graph $g$, provided that $g$ is valid. (Naturally, if $g$ is not valid, every possible move is appropriate as $g$ will eventually be rejected anyway.) To identify such a move, the parser needs criteria that it can check by inspecting the rest graph. These criteria should preferably only require a fixed number of patterns to be checked, in order to ensure that an appropriate move can be selected in constant time. While the desired patterns will obviously have to depend on $\Gamma$, they should be computable from the grammar by the parser generator. Such criteria do exist only if the dCFA is conflict-free and if $\Gamma$ has the free edge choice property in a sense to be made precise in this section. Thus, in contrast to the pure ASR parser, which works for every HR grammar, the resulting predictive shift-reduce parser exists only for a subset of all HR grammars, i.e., the parser generator may fail to construct a parser, reporting the existence of a conflict or failing to have the free edge choice property instead.

For the following considerations, suppose that the ASR parser is in the process of parsing a valid input graph $g$ and has reached a configuration $\mathcal{S} \cdot g^{\prime}$, but has not yet processed the rest graph $g^{\prime \prime}$ of $g$ where $g \bowtie g^{\prime} g^{\prime \prime} .{ }^{6}$ The top of $\mathcal{S}$ is $\operatorname{top}(\mathcal{S})=Q^{\tau}$ with a dCFA state $Q$ and an input binding $\tau$.

The parser must now choose between shift and reduce moves until the input graph has been accepted or no further move is possible. Shift moves are caused by transitions leaving $Q$, and reduce moves by items within $Q$ with a dot at the end of their right-hand side. Let us call such an item a reduce item. Each

\footnotetext{
${ }^{6}$ Note that we can represent the rest graph by any permutation of $g^{\prime \prime}$ since none of its literals have been processed by the parser yet.
} 
transition and each reduce item is called a trigger that causes the corresponding move. Note that acceptance is also caused by a reduce item, which is the only item in the accepting state $Q_{\mathrm{A}}$.

We now describe a decision procedure which inspects the rest graph $g^{\prime \prime}$ to select the trigger that causes an appropriate move, i.e., a move which turns the parser into a new configuration from which it can still reach an accepting configuration by reading the remaining rest graph. Let us call a sequence of moves that ends in an accepting configuration a successful sequence, even if it does not process the entire rest graph. Thm. 8.6 states that such a sequence always exists when the parser has reached $\mathcal{S} \cdot g^{\prime}$. The decision procedure must thus select a trigger that causes the first move of a successful sequence that processes the entire rest graph.

The idea for selecting the right trigger is as follows: Suppose that the rest graph $g^{\prime \prime}$ is not yet empty. The procedure now checks for each trigger whether $g^{\prime \prime}$ contains a literal $\boldsymbol{e}$ that may be processed next by some successful sequence caused by this trigger. There must be a trigger with this property since $g$ is valid. If this trigger is the only possible one that causes a successful sequence that eventually reads $\boldsymbol{e}$, this trigger must be the one causing the right move; the parser thus selects this trigger. In the following, we will show that this idea makes an effective decision procedure if the dCFA is conflict-free.

Let us consider more closely when a literal is processed next by a successful sequence caused by a trigger. If the trigger is a transition, this literal is just the one that is processed by the corresponding shift move. If the trigger, however, is a reduce item, it must be the one processed by the first shift move in the move sequence following the reduce move. This shift move may of course not be the first move of the sequence, as it can be preceded by further reduce moves.

Suppose now that the parser has processed the input graph entirely, i.e. the rest graph $g^{\prime \prime}$ is empty. The procedure then checks for each reduce item whether there is a successful sequence that consists of reduce moves only. The parser then selects any reduce item that causes such a successful sequence.

We will now discuss the decision procedure more precisely. To this end, we consider all successful sequences caused by a trigger. Recall that we assume that the parser has reached configuration $\mathcal{S} \cdot g^{\prime}$ with $\operatorname{top}(\mathcal{S})=Q^{\tau}$.

Suppose the trigger is a transition $\operatorname{tr}=\left(Q \stackrel{(e, \mu)}{\longrightarrow} Q^{\prime}\right)$ of the dCFA. Def. 8.1 implies that the shift move induced by $t r$ is $\mathcal{S} \cdot g^{\prime} \models_{t r} \mathcal{S} \bar{e} Q^{\prime \prime} \cdot g^{\prime} \overline{\boldsymbol{e}}$ for an appropriate literal $\overline{\boldsymbol{e}} \in \operatorname{Lit}_{\mathcal{T}}$ and state $Q^{\prime \prime}$. And by Thm. 8.6, there is a graph $v \in \mathcal{G}_{\mathcal{T}}$ such that $\mathcal{S} \overline{\boldsymbol{e}} Q^{\prime \prime} \cdot g^{\prime} \overline{\boldsymbol{e}} \models^{*} \mathcal{S}_{\mathrm{A}} \cdot g^{\prime} \overline{\boldsymbol{e}} v$ with $\operatorname{top}\left(\mathcal{S}_{\mathrm{A}}\right)=Q_{\mathrm{A}}$. This means that the parser accepts $g^{\prime} \overline{\boldsymbol{e}} v$ or, in other words, $\overline{\boldsymbol{e}} v$ is the graph processed by this successful sequence. Let us denote the set of all graphs processed by any successful sequence caused by $t r$ as $\operatorname{Success}\left(Q^{\tau}, g^{\prime}, t r\right)$.

Suppose now that the trigger is a reduce item it $=\langle\boldsymbol{A} \rightarrow \varrho ., \sigma\rangle \in Q$. Def. 8.1 implies that the reduce move induced by it is $\mathcal{S} \cdot g^{\prime} \models_{i t} \mathcal{S}^{\prime} \boldsymbol{A}^{\tau \circ \sigma} Q^{\prime} \cdot g^{\prime}$ with an appropriate parse stack $\mathcal{S}^{\prime}$ and state $Q^{\prime}$. And by Thm. 8.6, there is a graph $v \in \mathcal{G}_{\mathcal{T}}$ such that $\mathcal{S}^{\prime} \boldsymbol{A}^{\tau \circ \sigma} Q^{\prime} \cdot g^{\prime} \models^{*} \mathcal{S}_{\mathrm{A}} \cdot g^{\prime} v$ with $\operatorname{top}\left(\mathcal{S}_{\mathrm{A}}\right)=Q_{\mathrm{A}}$. 
This means that $v$ is the graph processed by this successful sequence. Let us denote the set of all graphs processed by any successful sequence caused by it as $\operatorname{Success}\left(Q^{\tau}, g^{\prime}, i t\right)$.

Before utilizing the sets $\operatorname{Success}\left(Q^{\tau}, g^{\prime}, t\right)$ for a trigger $t$, let us introduce some terminology. For a graph $h=\boldsymbol{e}_{1} \cdots \boldsymbol{e}_{n}$ with $n>0$ literals, let First $(h)=$ $\boldsymbol{e}_{1}$ be the first literal of $h$. In the special case $n=0$, we let First $(\varepsilon)=\$$ where the special symbol $\$$ indicates that there are no literals at all. For a set $S \subseteq \mathcal{G}_{\Sigma}$ of graphs, let First $(S)=\{$ First $(h) \mid h \in S\}$.

For a trigger $t$, now consider the set

$$
\text { First }\left(\operatorname{Success}\left(Q^{\tau}, g^{\prime}, t\right)\right) \text {. }
$$

This set contains all literals that can be processed next by successful sequences caused by $t$, and it contains $\$$ if there is a successful sequence caused by $t$ without any shift move. The decision procedure outlined above thus has to select the trigger $t$ such that First $\left(\operatorname{Success}\left(Q^{\tau}, g^{\prime}, t\right)\right)$ contains a literal of the rest graph, or $\$$ if $g^{\prime \prime}=\varepsilon$. However, this does not make a practical decision procedure as these sets are usually infinite. We turn them into finite sets by mapping their members to pseudo-literals as described next.

Note first that every node in any literal of any of these sets falls into one of three categories: It is either (1) a node assigned to a parameter of $Q$ by $\tau,(2)$ a node not occurring in $X\left(g^{\prime}\right)$, or $(3)$ a node in $X\left(g^{\prime}\right)$ not assigned to a parameter of $Q$ by $\tau$. We now define a function that maps nodes of category (1) to their corresponding parameter, nodes of category (2) to '-', and all others to ' $\bullet$ '.

$$
f_{Q}^{\tau, g^{\prime}}(x)= \begin{cases}y & \text { if there exists } y \in \operatorname{params}(Q) \text { such that } \tau(y)=x \\ - & \text { if } x \notin X\left(g^{\prime}\right) \\ \bullet & \text { otherwise }\end{cases}
$$

We extend function $f_{Q}^{\tau, g^{\prime}}$ to literals and sets of literals in the obvious way. Literals are thus turned into pseudo-literals, which are similar to literals, but may be attached to '-' and ' $\bullet$ ' instead of nodes. ${ }^{7}$

Function $f_{Q}^{\tau, g^{\prime}}$ applied to First $\left(\operatorname{Success}\left(Q^{\tau}, g^{\prime}, t\right)\right)$ turns this set into a finite set, because the number of terminal labels and the number of parameters in $Q$ are finite. However, this set cannot be computed from the HR grammar alone as it depends on $g^{\prime}$. Recall that the input binding $\tau$ is uniquely determined by $g^{\prime}$ because the dCFA approves $g^{\prime}$ by $\varepsilon Q_{0}^{\iota} \mid \approx{ }^{*} g^{\prime} \bullet Q^{\tau}$ and the dCFA is deterministic. To simplify things, let us define the finite set

$$
\text { Follow }(Q, t):=\bigcup_{g^{\prime} \in \mathcal{G}_{\Sigma}} f_{Q}^{\tau, g^{\prime}}\left(\operatorname{First}\left(\operatorname{Success}\left(Q^{\tau}, g^{\prime}, t\right)\right)\right)
$$

by building the union over all terminal graphs $g^{\prime}$. Clearly, only the graphs $g^{\prime}$ approved by the dCFA as mentioned above contribute to this set. This set

\footnotetext{
${ }^{7}$ Note that these pseudo-literals are a generalized version of those introduced in Sect. 6.
} 
just depends on the dCFA state $Q$ and one of its triggers $t$, and is thus static information independent of the input graph. While Follow $(Q, t)$ cannot directly be computed using (9.1), one can compute it by analyzing the dCFA in a way very similar to the computation of the follower symbols for string grammars (Sect. 3).

Example 9.1. Consider the dCFA for the tree-generating grammar in Fig. 10 and in particular its state $Q_{4}$, which has triggers $t r$ and $i t: t r$ is the transition from $Q_{4}$ to $Q_{3}$, and it is the reduce item $\langle T(y) \rightarrow T(y) e(y, z) T(z)$., $[y / a, z / b]\rangle$. $Q_{4}$ has the parameters $a$ and $b$. Function $f_{Q_{4}}^{\tau, g^{\prime}}$, when applied to nodes, thus maps into the set $\{-, \bullet, a, b\}$. In fact

$$
\begin{aligned}
\text { Follow }\left(Q_{4}, t r\right) & =\{e(b,-)\} \\
\text { Follow }\left(Q_{4}, i t\right) & =\{e(a,-), e(\bullet,-), \$\}
\end{aligned}
$$

It is clear that any successful sequence caused by transition $t r$ must begin with a shift move and that the read literal must match edge $e(b, c)$, which is ascribed to the transition. However, the "new" parameter $c$ is mapped to -.

Reduce item it can cause a successful sequence without any shift move, indicated by $\$$. To see this, consider, e.g., a parse stack $\mathcal{S}$ with $\operatorname{top}(\mathcal{S})=Q_{4}^{\tau}$ and $\operatorname{graph}(\mathcal{S})=\operatorname{root}(1) T(1) e(1,2) T(2)$. The reduce move will yield a stack $\operatorname{root}(1) T(1)$, which can be further reduced to $Z()$.

Moreover, $e(a,-)$ and $e(\bullet,-)$ indicate that the literal read next must be an $e$-literal attached to node $\tau(a)$ or to any node that has been processed already, but that is not kept track of by a parameter in $Q_{4}$, indicated by $\bullet$, and a node that has not yet been processed, indicated by -.

Now let $\boldsymbol{e}$ be a literal of the rest graph $g^{\prime \prime}$. The definition of Follow $(Q, t)$ implies that

$$
f_{Q}^{\tau, g^{\prime}}(e) \in \operatorname{Follow}(Q, t)
$$

is a necessary and easily verifiable condition for $\boldsymbol{e}$ to be a literal that can be processed next by a successful sequence caused by trigger $t$. Similarly, $\$ \in \operatorname{Follow}(Q, t)$ can be used to check whether $t$ can cause a successful sequence without any shift move. But this information is not yet sufficient to make an effective decision procedure. To see this, recall that function $f_{Q}^{\tau, g^{\prime}}$ may map many literals to the same pseudo-literal. Moreover, Follow $(Q, t)$ contains the pseudo-literals of any literal that may be read next in some successful sequence, not necessarily only those that process the rest graph $g^{\prime \prime}$ entirely. As a consequence, (9.2) does not yet make sure that $t$ is the right trigger. However, if we notice somehow - and additionally to (9.2) - that $\boldsymbol{e}$ can never be processed by any successful sequence not caused by $t$, we know for certain that $t$ is the only candidate for the right trigger. This observation leads the way to an effective procedure for selecting the right trigger.

Let us determine which literals can be processed by a successful sequence caused by a trigger $t$. We are not only interested in the literals that are processed first, but also in those literals that are processed eventually. Instead of a function 
First, we will use a function Any which is defined as follows: For a graph $h=\boldsymbol{e}_{1} \cdots \boldsymbol{e}_{n}$ with $n>0$ literals, let $\operatorname{Any}(h)=\left\{\boldsymbol{e}_{1}, \ldots, \boldsymbol{e}_{n}\right\}$ the set of all of its literals. For the empty graph, let $\operatorname{Any}(\varepsilon)=\{\$\}$. For a set $S \subseteq \mathcal{G}_{\Sigma}$ of graphs, let $\operatorname{Any}(S)=\bigcup_{h \in S} \operatorname{Any}(h)$. We then define the finite set

$$
\text { Follow }^{*}(Q, t):=\bigcup_{g^{\prime} \in \mathcal{G}_{\Sigma}} f_{Q}^{\tau, g^{\prime}}\left(\operatorname{Any}\left(\operatorname{Success}\left(Q^{\tau}, g^{\prime}, t\right)\right)\right) \text {. }
$$

Note the close resemblance to (9.1); the only difference is the use of Any instead of First, i.e., Follow ${ }^{*}(Q, t)$ contains the $f_{Q}^{\tau, g^{\prime}}$-images of all literals that occur eventually in some graph processed by a successful sequence caused by $t$, and it contains $\$$ if there is a successful sequence caused by $t$ that does not contain any shift move.

Again, this definition cannot be used for computing Follow $^{*}(Q, t)$ directly, but one can compute it by analyzing the dCFA in a similar way as for Follow $(Q, t)$.

Example 9.2. We continue Example 9.1 and consider again the dCFA for the tree-generating grammar shown in Fig. 10 and in particular its state $Q_{4}$ with its two triggers $t r$ and $i t$. In addition to

$$
\begin{aligned}
& \text { Follow }\left(Q_{4}, t r\right)=\{e(b,-)\} \\
& \text { Follow }\left(Q_{4}, i t\right)=\{e(a,-), e(\bullet,-), \$\}
\end{aligned}
$$

we have

$$
\begin{aligned}
& \text { Follow }^{*}\left(Q_{4}, t r\right)=\{e(b,-), e(\bullet,-), e(-,-)\} \\
& \text { Follow }^{*}\left(Q_{4}, i t\right)=\{e(a,-), e(\bullet,-), e(-,-), \$\}
\end{aligned}
$$

We can see that any literal that matches the only pseudo-literal $e(b,-)$ in Follow $\left(Q_{4}, t r\right)$ can never be processed in any successful sequence caused by $i t$, even if the rest graph contains literals matching the pseudo-literals $e(a,-)$ or $e(\bullet,-)$, which are members of Follow $\left(Q_{4}, i t\right)$. This can be concluded from the fact that $e(b,-)$ does not occur in Follow $^{*}\left(Q_{4}, i t\right)$. As a consequence, it cannot be the right trigger if we find a literal that matches $e(b,-)$.

However, we can see that a literal that matches $e(\bullet,-) \in$ Follow $\left(Q_{4}, i t\right)$ may indeed be processed later when transition $t r$ is chosen. The existence of a literal matching any pseudo-literal in Follow $\left(Q_{4}, i t\right)$ does thus not help to eliminate $t r$ from the candidates of right triggers.

As a consequence, a procedure can reliably predict the next move in state $Q_{4}$ by first checking whether there is a rest graph literal $\boldsymbol{e}$ with $f_{Q_{4}}^{\tau, g^{\prime}}(\boldsymbol{e})=e(b,-)$. If there is such a literal, $t r$ is guaranteed to be the right trigger because $e(b,-) \notin$ Follow $^{*}\left(Q_{4}, i t\right)$. If such a literal, however, does not exist, $t r$ cannot be the right trigger. One then checks whether the rest graph contains any literal $e^{\prime}$ that matches a pseudo-literal of Follow $\left(Q_{4}, i t\right)$, i.e., with $f_{Q_{4}}^{\tau, g^{\prime}}\left(\boldsymbol{e}^{\prime}\right) \in$ Follow $\left(Q_{4}, i t\right)$. If there is such a literal, one chooses the reduce move caused by $i t$. If there is no 
such $\boldsymbol{e}^{\prime}$, it is guaranteed that there is no successful sequence caused by it that processes the rest graph entirely, and the parser can terminate with a failure.

This example motivates that one must compare the Follow and Follow ${ }^{*}$ sets of the different triggers and that one must determine which trigger should be considered first when looking for rest graph literals that match any pseudoliterals in the Follow set of this trigger:

Definition 9.3. A trigger $t$ precedes a trigger $t^{\prime}$, written $t \prec t^{\prime}$, if $t$ and $t^{\prime}$ are triggers of the same dCFA state $Q, t \neq t^{\prime}$, and Follow $w^{*}(Q, t) \cap$ Follow $\left(Q, t^{\prime}\right) \neq \varnothing$.

Note that $\prec$ is not an ordering because it is in general not transitive. But $t \prec t^{\prime}$ indicates that one must check $t$ prior to $t^{\prime}$. However, $t \prec t^{\prime}$ does not help to find an order if there is a $\prec$-chain $t \prec t^{\prime} \prec \cdots \prec t$. This motivates the definition of conflicting triggers. We will see in the following that an effective decision procedure for identifying the right trigger requires conflict-freeness:

Definition 9.4. Let $Q$ be a dCFA state and $T_{Q}$ the set of its triggers. A subset $T \subseteq T_{Q}$ is in conflict if there is a sequence $t_{1} \prec t_{2} \prec \cdots \prec t_{k} \prec t_{1}$ with $T=\left\{t_{1}, t_{2}, \ldots, t_{k}\right\} . Q$ is conflict-free if no subset of its triggers is in conflict.

If a dCFA state is conflict-free, one can sort the triggers so that their order respects $\prec$, which will be necessary for the effective decision procedure:

Lemma 9.5. For every conflict-free dCFA state $Q$, there is an ordered sequence $t_{1}, \ldots, t_{n}$ of its triggers such that Follow $\left(Q, t_{i}\right) \cap$ Follow $^{*}\left(Q, t_{j}\right)=\varnothing$ for every pair of indices $i, j$ with $i<j$.

Proof. Let $T_{Q}$ be the set of triggers of $Q . T_{Q}$ can be considered as a directed graph with triggers acting as nodes and having an edge from $t$ to $t^{\prime}$ iff $t \prec t^{\prime}$. A cycle in $T_{Q}$ would indicate a conflict of the members of the cycle. Therefore, one can sort the transitions topologically into an ordered sequence $t_{1}, \ldots, t_{k}$ such that $T_{Q}=\left\{t_{1}, \ldots, t_{k}\right\}$ and $t_{i} \prec t_{j}$ implies $i<j$ for every pair of indices $i, j$. As a consequence, $j<i$ implies $t_{i} \nprec t_{j}$, which is equivalent to $\operatorname{Follow}\left(Q, t_{j}\right) \cap$ Follow $^{*}\left(Q, t_{i}\right)=\varnothing$.

Before we will use this ordered sequence of triggers to devise an efficient procedure that reliably identifies an appropriate trigger for the next move together with the yet unread literal to be shifted if the selected trigger is a transition, we show that the number of moves that an ASR parser needs to check the validity of a graph depends indeed linearly on the size of the graph. We will need this result later in the correctness proof for PSR parsers and for discussing their performance.

Lemma 9.6. If no $Q \in \mathcal{Q}$ has conflicts, there is a constant $c \in \mathbb{N}$ so that $n<c \cdot\left(\left|g_{n}\right|-\left|g_{0}\right|+1\right)$ for every sequence $Q_{0}^{\iota} \cdot \varepsilon \models{ }^{*} \mathcal{S}_{0} \cdot g_{0} \models \mathcal{S}_{1} \cdot g_{1} \models \cdots \models \mathcal{S}_{n} \cdot g_{n}$ of $A S R$ moves where $\mathcal{S}_{0}$ is a prefix of $\mathcal{S}_{i}$ for $i=1, \ldots, n$. 
Proof. Let us assume that none of the dCFA states has a conflict. We prove the lemma by induction on $\left|g_{n}\right|-\left|g_{0}\right|$.

For $g_{n}=g_{0}$, let us assume the contrary, i.e., there is, for any $m \in \mathbb{N}$, a sequence $Q_{0}^{\iota} \cdot \varepsilon \models{ }^{*} \mathcal{S}_{0} \cdot g_{0} \models \mathcal{S}_{1} \cdot g_{0} \models \cdots \models \mathcal{S}_{n} \cdot g_{0}$ where $\mathcal{S}_{0}$ is a prefix of $\mathcal{S}_{i}$ for $i=1, \ldots, n$, and $n \geq m$. Let $\operatorname{top}\left(\mathcal{S}_{i}\right)=\bar{Q}_{i}^{\tau_{i}}$ with dCFA states $\bar{Q}_{i} \in \mathcal{Q}$ and input bindings $\tau_{i}$ for $i=1, \ldots, n$. All moves in $\mathcal{S}_{0} \cdot g_{0} \models \mathcal{S}_{1} \cdot g_{0} \models \cdots \models \mathcal{S}_{n} \cdot g_{0}$ are reduce moves, and by the definition of reduce moves, we have $\tau_{i}(X) \subseteq \tau_{0}(X)$ for $i=1, \ldots, n$. Because $\mathcal{Q}$ and $\tau_{0}(X)$ are finite and because $n \geq m$, there must be some $j, k$ with $0 \leq j<k \leq n$ and $\bar{Q}_{j}^{\tau_{j}}=\bar{Q}_{k}^{\tau_{k}}$, i.e., the subsequence of moves $\mathcal{S}_{j} \cdot g_{0} \models \mathcal{S}_{j+1} \cdot g_{0} \models \cdots \models \mathcal{S}_{k} \cdot g_{0}$ forms a cycle. Because the grammar is reduced, there must be a chance to leave the cycle, i.e., there is an index $\hat{\imath}$ with $j \leq \hat{\imath} \leq k$ such that $\bar{Q}_{\hat{\iota}}$ must have at least two outgoing transitions: $t$ belongs to the cycle and goes into the next state of the cycle whereas $t^{\prime}$ leads out of the cycle. Let us choose any $\ell \in$ Follow $\left(\bar{Q}_{\hat{\iota}}, t^{\prime}\right)$, i.e., $\ell$ is either a pseudo-literal or $\ell=\$$. In either case, $\ell \in$ Follow $\left(\bar{Q}_{\hat{\imath}}, t\right)$, too, because $\bar{Q}_{\hat{\iota}}^{\tau_{\hat{\imath}}}$ can be reached again when using transition $t$. And because Follow $\left(\bar{Q}_{\hat{\imath}}, t\right) \subseteq$ Follow $^{*}\left(\bar{Q}_{\hat{\imath}}, t\right)$ and Follow $\left(\bar{Q}_{\hat{\imath}}, t^{\prime}\right) \subseteq$ Follow $^{*}\left(\bar{Q}_{\hat{\imath}}, t^{\prime}\right)$, we can conclude $t \prec t^{\prime} \prec t$, i.e., $\bar{Q}_{\hat{\imath}}$ has a conflict, in contradiction to the assumption that none of the dCFA states has conflicts.

For $\left|g_{n}\right|-\left|g_{0}\right|>0$, we consider any sequence

$$
Q_{0}^{\iota} \cdot \varepsilon \models^{*} \mathcal{S}_{0} \cdot g_{0} \models \mathcal{S}_{1} \cdot g_{1} \models \cdots \models \mathcal{S}_{n} \cdot g_{n}
$$

of ASR moves where $\mathcal{S}_{0}$ is a prefix of $\mathcal{S}_{i}$ for $i=1, \ldots, n$. Let $k$ be the index where $\left|g_{k}\right|+1=\left|g_{k+1}\right|=\left|g_{n}\right|$, i.e., there is a literal $\boldsymbol{e}$ so that $g_{k} \boldsymbol{e}=g_{k+1}=$ $g_{k+1}=\cdots=g_{n}$. Sequence (9.4) can thus be written in two ways:

$$
\begin{aligned}
& Q_{0}^{\iota} \cdot \varepsilon \models{ }^{*} \mathcal{S}_{0} \cdot g_{0} \models \mathcal{S}_{1} \cdot g_{1} \models \cdots \models \mathcal{S}_{k} \cdot g_{k} \\
& Q_{0}^{\iota} \cdot \varepsilon \models{ }^{*} \mathcal{S}_{k+1} \cdot g_{k} \boldsymbol{e} \models \mathcal{S}_{k+2} \cdot g_{k} \boldsymbol{e} \models \cdots \models \mathcal{S}_{n} \cdot g_{k} \boldsymbol{e}
\end{aligned}
$$

By the induction hypothesis, we have $\left.k<c \cdot\left(\left|g_{k}\right|-\left|g_{0}\right|+1\right)=c\left(\left|g_{n}\right|-\mid g_{0}\right]\right)$ for (9.5). And by the same arguments as for the case $g_{n}=g_{0}$, we have $n-k-1<c$ for (9.6). Therefore, $\left.n \leq c \cdot\left(\left|g_{n}\right|-\mid g_{0}\right]+1\right)$.

The following corollary is an immediate consequence of the previous lemma by choosing $\mathcal{S}_{0}=Q_{0}^{\iota}, \mathcal{S}_{n}=\mathcal{S}, g_{0}=\varepsilon$, and $g_{n}=g$ :

Corollary 9.7. If no $Q \in \mathcal{Q}$ has conflicts, there is a constant $c \in \mathbb{N}$ so that $n<c \cdot|g|+c$ for every sequence $Q_{0}^{\iota} \cdot \varepsilon \models^{n} \mathcal{S} \cdot g$ of ASR moves.

This linear bound on the number of parser moves is in fact a consequence of the required absence of conflicts. Note that there is no such bound on the length of a derivation $\boldsymbol{Z} \Rightarrow^{*} g$ for an arbitrary HR grammar.

We are now going to devise the procedure SelectTrigger below that reliably identifies an appropriate trigger for the next move together with the yet unread literal to be shifted if the selected trigger is a transition. This procedure will control the parser and what parser move is executed next. We will show that 
this procedure is effective as well as efficient for certain HR grammars. These are grammars whose dCFA states are all conflict-free and that have an additional property, the free edge choice property, which we are going to define below. SelectTrigger is effective in the sense that it will always select an appropriate move that leads to a (successful) sequence of parser moves ending in an accepting configuration if the input graph is valid. It is also efficient as constant time suffices for computing the results.

The latter might come as a surprise because SelectTrigger must perform a kind of graph matching task. The procedure is in fact called after each move of the ASR parser (which we will then extend to the PSR parser) when it is in a configuration $\mathcal{S} \cdot g^{\prime}$ with $\operatorname{top}(\mathcal{S})=Q^{\tau}$ with a dCFA state $Q \in \mathcal{Q}$ and an input binding $\tau$. SelectTrigger must then select the appropriate trigger $t$ for $Q$ by checking whether the unread part $g^{\prime \prime}$ of the input graph contains a literal whose $f_{Q}^{\tau, g^{\prime}}$-image is a member of Follow $(Q, t)$ as it has been discussed above. SelectTrigger in fact uses a simpler criterion and looks for literals of $g^{\prime \prime}$ that are attached to certain nodes determined by some pseudo-literal $\ell \in$ Follow $(Q, t)$. We will show later that this simpler criterion still makes the procedure effective if the grammar has the free edge choice property. The look-up criterion uses the binary relation fits defined as follows:

Definition 9.8. Let $\ell=a\left(y_{1}, \ldots, y_{k}\right)$ be a pseudo-literal and $\boldsymbol{e} \in \mathrm{Lit}_{\mathcal{T}}$ a literal. Furthermore, let $Q$ be a dCFA state and $\tau$ an input binding. We say that $\boldsymbol{e}$ fits $\ell$ in $Q^{\tau}$, written $\boldsymbol{e}$ fits $_{Q}^{\tau} \ell$, if and only if $\boldsymbol{e}=a\left(x_{1}, \ldots, x_{k}\right)$ and $x_{i}=\tau\left(y_{i}\right)$ for each $i=1, \ldots, n$ with $y_{i} \in \operatorname{params}(Q)$.

Note that, given a pseudo-literal $\ell$, there is no requirement on the attached nodes of $\boldsymbol{e}$ that correspond to any $y_{i}$ which are not parameters of $Q$. Note also that, by the definition of $\operatorname{fits}_{Q}^{\tau}$ and $f_{Q}^{\tau, g^{\prime}}, \boldsymbol{e}$ fits ${ }_{Q}^{\tau} f_{Q}^{\tau, g^{\prime}}(\boldsymbol{e})$ holds for every literal $\boldsymbol{e}$, graph $g^{\prime}$, dCFA state $Q$, and input binding $\tau$.

SelectTrigger below shows the pseudo-code of the decision procedure using the fits relation. It returns the appropriate trigger and, also, the literal to be read next, or $\$$ if $g^{\prime \prime}=\varepsilon$; it returns 'failure' if it is guaranteed that there is no successful sequence processing $g^{\prime \prime}$ entirely. Note that the procedure requires an ordered sequence of all triggers as described in Lemma 9.5, i.e., it does not work if there are conflicting triggers.

We will now show the effectiveness of SelectTrigger in the sense that it always returns the appropriate trigger together with the literal to be read next. It is clear from line 1 that the procedure cannot work if any state of the dCFA has conflicts. But the grammar must have yet another property, which we are going to define next. The rationale behind this property is the following: There may be several unread literals that may be read by a transition selected as the appropriate trigger. Some of those literals may lead to a successful parse whereas others do not. The free edge choice property simply requires that it does not matter which of the fitting literals is selected. The property additionally requires that the simpler criterion based on the fits relation (Def. 9.8) may in fact be used. 


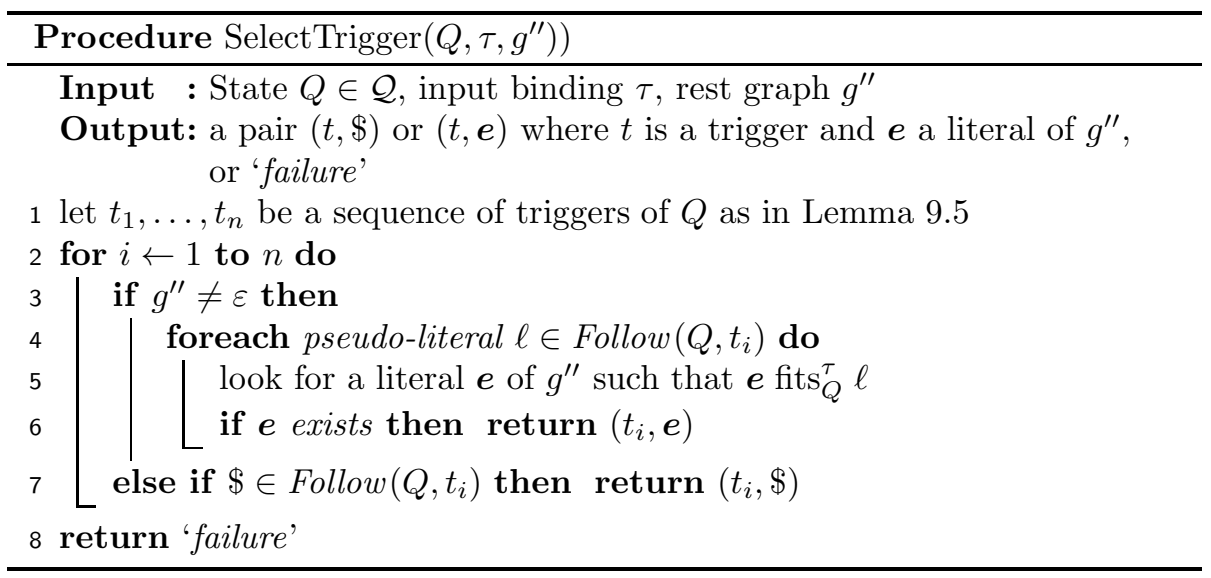

Definition 9.9 (Free edge choice property). Let $g^{\prime}, g^{\prime \prime} \in \mathcal{G}_{\mathcal{T}}$ be any graphs, $g^{\prime \prime} \neq \varepsilon, \mathcal{S} \cdot g^{\prime}$ a configuration reached by the ASR parser, $\operatorname{top}(\mathcal{S})=Q^{\tau}$ where $Q$ is a dCFA state and $\tau$ an input binding. The return value $(t, \boldsymbol{e})$ of SelectTrigger $\left(Q, \tau, g^{\prime \prime}\right)$ is called useful if

(i) $f_{Q}^{\tau, g^{\prime}}(\boldsymbol{e}) \in$ Follow $(Q, t)$, and

(ii) there is a graph $h \in \mathcal{G}_{\mathcal{T}}$ such that $e h \bowtie g^{\prime \prime}$ and

$$
\mathcal{S} \cdot g^{\prime} \models_{t} \mathcal{S}^{\prime} \cdot g^{\prime} \boldsymbol{e} \models^{*} \mathcal{S}_{\mathrm{A}} \cdot g^{\prime} \boldsymbol{e h}
$$

with $\operatorname{top}\left(\mathcal{S}_{\mathrm{A}}\right)=Q_{\mathrm{A}}$ if

$$
\mathcal{S} \cdot g^{\prime} \equiv_{t} \mathcal{S}^{\prime} \cdot g^{\prime} \boldsymbol{e}^{\prime} \models^{*} \mathcal{S}_{\mathrm{A}} \cdot g^{\prime} \boldsymbol{e}^{\prime} h^{\prime}
$$

for any literal $\boldsymbol{e}^{\prime}$ and graph $h^{\prime} \in \mathcal{G}_{\mathcal{T}}$ so that $\boldsymbol{e}^{\prime} h^{\prime} \bowtie g^{\prime \prime}$.

The grammar is said to have the free edge choice property if the return value of SelectTrigger $\left(Q, \tau, g^{\prime \prime}\right)$ is always useful.

This property is in fact similar to the property defined in [12] under the same name for PTD parsing whereas we now need this property for PSR parsing. There are sufficient criteria that can be used by static grammar analysis that guarantee that a grammar has the free edge choice property and that have been realized in the Grappa tool (see below). A discussion of how this property can effectively be tested is out of scope of the present paper, however.

We will show below that SelectTrigger returns reliable results only for grammars with the free edge choice property. We thus extend Assumption 7.6:

Assumption 9.10. For the remainder of the paper, we further assume that every state $Q$ of the dCFA is conflict-free and that $\Gamma$ has the free edge choice property. 
The following lemma states that SelectTrigger, under this assumption, can reliably identify the unique right trigger together with the unread literal to be read next:

Lemma 9.11. Let $\mathcal{S} \cdot g^{\prime}$ be any configuration reached by the ASR parser and $\operatorname{top}(\mathcal{S})=Q^{\tau}$ where $Q$ is a dCFA state and $\tau$ an input binding.

For every graph $g^{\prime \prime} \in \mathcal{G}_{\mathcal{T}}$ such that $\mathcal{S} \cdot g^{\prime} \models^{*} \mathcal{S}_{\mathrm{A}} \cdot g^{\prime} g^{\prime \prime}$ with $\operatorname{top}\left(\mathcal{S}_{\mathrm{A}}\right)=Q_{\mathrm{A}}$, SelectTrigger $\left(Q, \tau, g^{\prime \prime}\right)$ returns a pair $(t, \boldsymbol{e})$ with the following properties:

- If $t=\langle\boldsymbol{S t a r t} \rightarrow \boldsymbol{Z} \cdot, \iota\rangle$ is the reduce item causing acceptance, then $\mathcal{S}=$ $\mathcal{S}_{\mathrm{A}}, e=\$$, and $g^{\prime \prime}=\varepsilon$.

- If $t \neq\langle\boldsymbol{S t a r t} \rightarrow \boldsymbol{Z} \cdot, \iota\rangle$ is any other reduce item, there is a stack $\mathcal{S}^{\prime}$ with

$$
\mathcal{S} \cdot g^{\prime} \models_{t} \mathcal{S}^{\prime} \cdot g^{\prime} \models^{*} \mathcal{S}_{\mathrm{A}} \cdot g^{\prime} g^{\prime \prime}
$$

and $g^{\prime \prime}=\varepsilon$ iff $e=\$$.

- If $t$ is a transition, then $\boldsymbol{e}$ is a literal of $g^{\prime \prime}$ and

$$
\mathcal{S} \cdot g^{\prime} \models_{t} \mathcal{S e} Q^{\prime} \cdot g^{\prime} \boldsymbol{e} \models^{*} \mathcal{S}_{\mathrm{A}} \cdot g^{\prime} \boldsymbol{e h}
$$

for some state $Q^{\prime}$ and a graph $h \in \mathcal{G}_{\mathcal{T}}$ with $g^{\prime \prime} \bowtie e h$.

Proof. Let $\mathcal{S} \cdot g^{\prime}, Q, \tau$, and $g^{\prime \prime}$ be as in the lemma. We distinguish three cases:

(1) $g^{\prime \prime}=\varepsilon$ and $Q=Q_{\mathrm{A}} \cdot Q_{\mathrm{A}}$ consists of just the reduce item it $=\langle$ Start $\rightarrow$ $Z \mathbf{Z}, \iota\rangle$ and $\$ \in$ Follow $\left(Q_{A}, i t\right)$. Thus SelectTrigger returns $(i t, \$)$, and the parser terminates by accepting $g^{\prime}$.

(2) $g^{\prime \prime}=\varepsilon$ and $Q \neq Q_{\mathrm{A}}$. There must be a nonempty sequence $s$ of reduce moves leading to $\mathcal{S}_{\mathrm{A}} \cdot g^{\prime}$. Because of $g^{\prime \prime}=\varepsilon$, the procedure returns $(t, \boldsymbol{e})=\left(t_{i}, \$\right)$ in line 7 , and $\$ \in$ Follow $\left(Q, t_{i}\right)$. In fact, $t_{i}$ is the only trigger in $Q$ with $\$ \in \operatorname{Follow}\left(Q, t_{i}\right)$. Otherwise, if $\$ \in \operatorname{Follow}\left(Q, t_{j}\right)$ for $j<i$, SelectTrigger would have returned $\left(t_{j}, \$\right)$. And if $\$ \in \operatorname{Follow}\left(Q, t_{j}\right)$ for $j>i$, we would have $\$ \in$ Follow $^{*}\left(Q, t_{j}\right)$, in contradiction to the construction of sequence $t_{1}, \ldots, t_{n}$. Therefore, $s$ must be caused by $t$ as in (9.9).

(3) $g^{\prime \prime} \neq \varepsilon$. There is a successful sequence since

$$
\mathcal{S} \cdot g^{\prime} \models^{*} \mathcal{S}_{\mathrm{A}} \cdot g^{\prime} g^{\prime \prime},
$$

which contains at least one shift move. Let $t_{j}$ be the trigger causing this particular sequence and $\overline{\boldsymbol{e}}$ the literal processed by its first shift move. Then

$$
f_{Q}^{\tau, g^{\prime}}(\overline{\boldsymbol{e}}) \in \operatorname{Follow}\left(Q, t_{j}\right) .
$$

SelectTrigger cannot return any pair $\left(t_{i}, \boldsymbol{e}^{\prime}\right)$ where $i<j$. To see this, let us assume the contrary, i.e., $g^{\prime \prime}$ contains a literal $e^{\prime}$ with $e^{\prime}$ fits $_{Q}^{\tau} \ell$ for some $\ell \in \operatorname{Follow}\left(Q, t_{i}\right)$. Because of the free edge choice property, in particular 
Def. 9.9(i), we can conclude that $f_{Q}^{\tau, g}\left(\boldsymbol{e}^{\prime}\right) \in \operatorname{Follow}\left(Q, t_{i}\right)$ and, therefore, $f_{Q}^{\tau, g}\left(\boldsymbol{e}^{\prime}\right) \notin$ Follow $^{*}\left(Q, t_{j}\right)$ by the construction of $t_{1}, \ldots, t_{n}$. But this means that $\boldsymbol{e}^{\prime}$ cannot be processed by any shift move in (9.11), in contradiction to (9.11) being a successful sequence.

Because of (9.12) and $\overline{\boldsymbol{e}} \operatorname{fits}_{Q}^{\tau} f_{Q}^{\tau, g^{\prime}}(\overline{\boldsymbol{e}})$, SelectTrigger can find $\overline{\boldsymbol{e}}$ in line 5 as soon as $t_{j}$ is selected in the outer for-loop. But $g^{\prime \prime}$ may also contain a different literal $\boldsymbol{e}$ that fits a pseudo-literal $\ell \in \operatorname{Follow}\left(Q, t_{j}\right)$, and SelectTrigger returns $\left(t_{j}, \boldsymbol{e}\right)$. Trigger $t_{j}$ may be either a reduce item or a transition. If it is a reduce item, (9.11) has in fact the form (9.9), which proves the lemma. But if $t_{i}$ is a transition, there is a successful sequence

$$
\mathcal{S} \cdot g^{\prime} \underset{\bar{t}_{j}}{\models} \mathcal{S}^{\prime} \cdot g^{\prime} \boldsymbol{e} \models^{*} \mathcal{S}_{\mathrm{A}} \cdot g^{\prime} \boldsymbol{e h}
$$

where $h \in \mathcal{G}_{\mathcal{T}}$ is a graph such that $\boldsymbol{e} h \bowtie g^{\prime \prime}$ because of (9.11) and the free edge choice property, in particular Def. 9.9(ii). This proves the lemma because (9.13) has the form (9.10).

SelectTrigger can now be used to predict the next move in every configuration reachable by the ASR parser. This leads to the predictive shift-reduce (PSR) parser, the main notion proposed in this paper, which is in fact the ASR parser equipped with SelectTrigger for selecting the next move:

Definition 9.12 (PSR Parser). A (PSR parser) configuration $\mathcal{S} \cdot g \mid \bar{g}$ is an ASR parser configuration $\mathcal{S} \cdot g$ together with a rest graph $\bar{g} \in \mathcal{G}_{\mathcal{T}} \cdot \mathcal{S} \cdot g \mid \bar{g}$ is accepting if $\bar{g}=\varepsilon$ and $\mathcal{S} \cdot g$ is an accepting ASR parser configuration.

A PSR move turns $\mathcal{S} \cdot g \mid \bar{g}$ into $\mathcal{S}^{\prime} \cdot g^{\prime} \mid \bar{g}^{\prime}$, written $\mathcal{S} \cdot g\left|\bar{g} \Vdash \mathcal{S}^{\prime} \cdot g^{\prime}\right| \bar{g}^{\prime}$, if

(i) SelectTrigger $(Q, \tau, \bar{g})$ returns $(t, e)$, where $\operatorname{top}(\mathcal{S})=Q^{\tau}$ for a state $Q \in \mathcal{Q}$ and input binding $\tau$,

(ii) $g^{\prime}=g \boldsymbol{e}$ if $t$ is a transition and $g^{\prime}=g$ otherwise,

(iii) $g \bar{g} \bowtie g^{\prime} \bar{g}^{\prime}$, and

(iv) $\mathcal{S} \cdot g \models_{t} \mathcal{S}^{\prime} \cdot g^{\prime}$ is an ASR move.

A PSR parser can reach a configuration $\mathcal{S}^{\prime} \cdot g^{\prime} \mid \bar{g}^{\prime}$ from $\mathcal{S} \cdot g \mid \bar{g}$ if $\mathcal{S} \cdot g \mid \bar{g} \Vdash^{*}$ $\mathcal{S}^{\prime} \cdot g^{\prime} \mid \bar{g}^{\prime}$. A PSR parser accepts a graph $g \in \mathcal{G}_{\mathcal{T}}$ if it can reach an accepting configuration $\mathcal{S} \cdot g^{\prime} \mid \varepsilon$ from $Q_{0}^{\iota} \cdot \varepsilon \mid g$.

We are now ready for the main result of this paper. It shows that a PSR parser, for an HR grammar satisfying Assumption 9.10, accepts a graph if and only if it is valid. Moreover, a PSR parser cannot run into dead ends, i.e., whenever a PSR parser fails, there cannot be a different sequence of parser moves that is successful:

Theorem 9.13. Let $Q_{0}^{\iota} \cdot \varepsilon\left|g \Vdash^{*} \mathcal{S} \cdot g^{\prime}\right| \bar{g}^{\prime}$ be any sequence of PSR moves. The PSR parser can reach an accepting configuration from $\mathcal{S} \cdot g^{\prime} \mid \bar{g}^{\prime}$ if and only if $g$ is valid. 
Proof. Let us assume any HR grammar satisfying Assumption 9.10, graph $g \in \mathcal{G}_{\mathcal{T}}$ and sequence $Q_{0}^{\iota} \cdot \varepsilon\left|g \Vdash^{n} \mathcal{S} \cdot g^{\prime}\right| \bar{g}^{\prime}$ of PSR moves.

To show the only-if direction, let us assume that the parser can reach an accepting configuration $\mathcal{S}^{\prime} \cdot g^{\prime \prime} \mid \varepsilon$ from $\mathcal{S} \cdot g^{\prime} \mid \bar{g}^{\prime}$. Then $\boldsymbol{Z} \Rightarrow^{*} g^{\prime \prime}$ follows from Thm. 8.6 and the fact that each sequence of PSR moves is also a sequence of ASR moves. Moreover, $g \bowtie g^{\prime \prime}$ follows from Def. 9.12(iii), i.e., $g \in \mathcal{L}(\Gamma)$.

To show the if direction, let us assume that $g$ is valid. We first show, as an auxiliary result, that the ASR parser can reach an accepting ASR parser configuration $\mathcal{S}^{\prime} \cdot g^{\prime \prime}$ from $\mathcal{S} \cdot g^{\prime}$ and $g^{\prime \prime} \bowtie g$ by induction on $n$. For $n=0$, the ASR parser can reach an accepting configuration $\mathcal{S}^{\prime} \cdot g^{\prime \prime}$ by Thm. 8.6. For $n>0$, there is a sequence

$$
Q_{0}^{\iota} \cdot \varepsilon\left|g \Vdash^{n-1} \mathcal{S}^{\prime \prime} \cdot h\right| \bar{h} \Vdash \mathcal{S} \cdot g^{\prime} \mid \bar{g}^{\prime}
$$

of PSR moves and, using the induction hypothesis, an accepting ASR parser configuration $\mathcal{S}^{\prime \prime} \cdot h^{\prime}$ that the ASR parser can reach from $\mathcal{S}^{\prime \prime} \cdot h$. The last PSR move in (9.14) uses SelectTrigger. Therefore, Lemma 9.11 applies, and the ASR parser can reach an accepting configuration $\overline{\mathcal{S}}^{\prime \prime} \cdot h^{\prime \prime}$ where $h^{\prime \prime} \bowtie g$. This proves our auxiliary result, which we use now to show the if direction. By using Lemma 9.11 again, the auxiliary result implies that every sequence of PSR moves starting at $Q_{0}^{\iota} \cdot \varepsilon \mid g$ either ends with an accepting configuration, or can be extended by yet another PSR move. This process can be repeated, but it must end eventually because there cannot be an infinite sequence of ASR moves and, thus, of PSR moves because of Cor. 9.7. This proves the if direction since the final configuration of this sequence must be an accepting one.

Note, however, that the parser is still nondeterministic, despite the fact that it chooses the trigger causing the next parser move for every configuration deterministically. The reason is that SelectTrigger does not uniquely determine the literal to be processed by the shift move to be made. For instance, the ASR parser moves shown in Fig. 15, which are also valid PSR parser moves, choose edge $e^{12}$ in the third move, but could have chosen $e^{13}$ instead, keeping $e^{12}$ for later. There are thus two different sequences of parser moves that both prove the validity of the given input graph, i.e., the PSR parser is nondeterministic. However, this nondeterminism is harmless as it does not make a difference when it comes to acceptance because of the free edge choice of the grammar.

The Grappa tool implemented by Mark Minas generates PSR parsers based on the construction of the dCFA and the analysis of the criteria for Assumption 9.10 outlined above. Table 1 summarizes results for some HR grammars. The columns under "Grammar" indicate the size of the grammar in terms of the maximal arity of nonterminals (A), number of nonterminals $(\mathrm{N})$, number of terminals $(\mathrm{T})$ and number of rules $(\mathrm{R})$. The columns under "dCFA" indicate the size of the generated dCFA in terms of the number of states (S), the overall number of items (I) and the number of transitions $(\Delta)$. The number of conflicting sets in the dCFA is shown in the column "Conflicts". The last column indicates whether the grammar satisfies the free edge choice property. Note that the PSR parser can successfully be generated for the grammars without any conflicts and satisfying the free edge choice property. For the others, 
the parser generator fails with a message pointing out the reason of the failure.

Table 1: PSR-Parsability of some HR grammars.

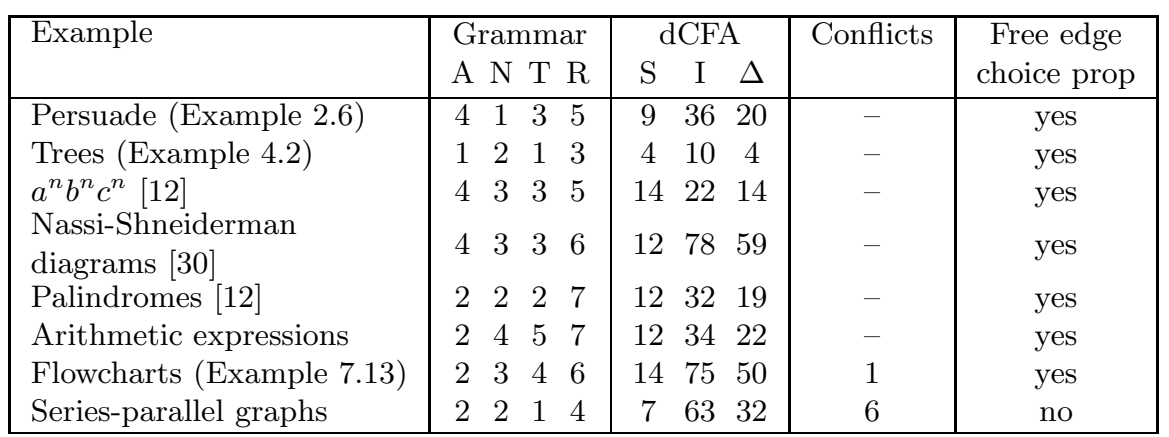

We refer the reader to [24, Sect. 6] for runtime measurements of PSR parsers that confirm that they run in linear time, for all practical purposes. Let us finally outline why this is the case for an HR grammar satisfying Assumption 9.10: The number of moves required by a PSR parser grows linearly with the number of literals of the input graph, i.e., its size, and independent of the graph being valid or not (Cor. 9.7). For each move, the parser must first call SelectTrigger and then execute the selected action. The latter part depends on the grammar only and is in particular independent of the size of the input graph, i.e., it always takes constant time.

Let us now discuss the efficiency of SelectTrigger, i.e., consider how long an invocation of this procedure takes. The number of triggers limits the number of how often the loop starting in line 2 is executed. This number is bounded by a constant that depends on the grammar only. The runtime of SelectTrigger is thus determined by the time required to execute line 5 . It must find a literal $\boldsymbol{e}$ in the rest graph such that it "fits" some member of Follow $\left(Q, t_{i}\right)$. Note that the size of the latter set is also bounded by a constant that depends on the grammar only.

We now consider any pseudo-literal in Follow $\left(Q, t_{i}\right)$, and argue that looking up a literal of the rest graph that fits this pseudo-literal always takes constant time if the input graph has been pre-processed in an appropriate way. Note that a certain number of attached nodes of such a literal is already fixed by the pseudo-literal via the fits $_{Q}^{\tau}$ relation. If no node is fixed, selecting such a literal is easy: One picks an arbitrary unread literal with the appropriate label. This must be the right choice because of the free edge choice property. If exactly one node is fixed, one picks any unread literal that is attached to this node. This can be done in constant time if each node maintains a list of all unread literals attached to this node. Such association lists can be set up prior to parsing in time $O(n+m)$ if the input graph has $n$ literals and $m$ nodes. And if two or more nodes are fixed, selecting any unread literal attached to these nodes can 
also be done in (worst case) constant time by maintaining association lists of yet unread literals for each of these node combinations and storing these association lists in hash tables that allow efficient lookup by these node combinations, for instance using Cuckoo Hashing [31], which supports (worst case) constant time access during parsing, and an expected linear time set-up once prior to parsing.

As a consequence, a PSR parser always has a (worst case) linear parsing time. It additionally needs a set-up of some search data structures in either worst case linear or expected linear time, though. Expected linear time processing is only needed if SelectTrigger must look up unread literals with two or more nodes fixed. But we have argued in [24, Sect. 5] that one can improve preprocessing even for many of those grammars: Even though SelectTrigger would have to look up unread literals with two or more nodes fixed, static analysis of the grammar may reveal that it is in fact sufficient to consider just one of these fixed nodes. The PSR parser for grammars with this property thus need not manage association lists in hash tables, which would require expected linear time for preprocessing, but can attach them to nodes, which requires linear time in the worst case for preprocessing. In fact, we had to particularly handcraft an HR grammar (the "blowball" grammar in [24]) that indeed requires hash tables for efficient parsing and thus preprocessing in expected linear time. All PSR parsers for grammars without conflicts in Table 1 need linear time in the worst case though.

\section{Conclusions}

We have devised a predictive shift-reduce (PSR) parsing algorithm for HR grammars, along the lines of SLR(1) string parsing, thus continuing the work begun in [14] by formalizing the construction of PSR parsers and proving its correctness. PSR parsing is somewhat complementary to predictive top-down (PTD) parsing that lifts SLL(1) parsing for string grammars to HR grammars [12]. Checking PSR-parsability is complicated enough, but easier than for PTD, as we do not need to consider HR rules that merge nodes of their lefthand sides, which is necessary for PTD parsing. PSR parsers also work more efficiently than PTD parsers: while PTD parsers require quadratic time in the worst case, PSR parsers run in linear time for all practical purposes; see the discussion in Sect. 9, and in particular Cor. 9.7. The reader is encouraged to download the Grappa generator of PTD and PSR parsers and to conduct own experiments. $^{7}$

\section{Related Work}

Much related work on graph parsing has been done for graph grammars based on context-free node replacement [16]. In these grammars, a node $v$ is replaced by a graph $R$, where embedding instructions specify what happens to

7 The Grappa tool is available at www.unibw.de/inf2/grappa; the examples mentioned in Table 1 can be found there as well. 
the edges incident in $v$; in general, such an edge can just be deleted, or turned around, or replicated and directed towards different nodes of $R$. Node replacement has greater generative power, but is difficult to handle for general embedding instructions. So papers on parsing for node replacement graph grammars restrict these instructions. The earliest ones (to our knowledge), by T. Pavlides, T.W. Pratt, and P. Della Vigna and C. Ghezzi, [32, 33, 7], appeared well before visual user interfaces supported input and processing of diagrams by computers. R. Franck [20] extended precedence string parsing to graphs, in order to implement a "two-dimensional programming language" based on Algol-68. W. Kaul corrected and extended this idea of parsing [25]. His parser is linear, and can cope with ambiguous grammars, but fails to parse some languages that are both PSR- and PTD-parsable, like the trees of Example 4.2.

A parsing algorithm following the idea of the well-known Cocke-YoungerKasami algorithm was proposed and investigated by C. Lautemann [27] who gave a sufficient condition under which this algorithm is polynomial. However, even if the condition is met, the degree of the polynomial depends on the grammar. The algorithm was recently refined by D. Chiang et al. [4], making it more practical but without changing its general characteristics. An alternative algorithm developed by W. Vogler in [34] and generalized by F. Drewes in [9] guarantees a cubic running time at the expense of employing a severe connectedness requirement. Due to this requirement it seems fair to say that this algorithm is mainly of theoretical interest. A promising approach for certain types of applications, especially for graph languages appearing in computational linguistics, has recently been proposed by S. Gilroy, A. Lopez, and S. Maneth [21]. This parsing algorithm applies to Courcelle's "regular" graph grammars [6] and runs in linear time.

Over the years, M. Flasiński and his group have developed top-down and bottom-up parsing techniques for pattern recognition $[17,18,19]$. The graph classes they consider are very restricted: rooted directed acyclic graphs with ordered nodes. Their parsers are also linear, but this is achieved by forbidding all concepts that make graph parsing essentially different from string parsing. According to our knowledge, another early attempt at $L R$-like graph parsers by H.J. Ludwigs [29] has never been completed.

G. Costagliola's positional grammars [5] are used to specify visual languages, but they can also describe certain HR languages. Although they are parsed in an LR-like fashion, many decisions are deferred until the parser is actually executed, in order to avoid complex analyses of the grammar when the parsers are generated. In contrast, the PSR parser generator implemented in the Grappa tool performs an elaborate static analysis of the grammar. It includes the detection of conflicts that prevent the parser from running into situations where, despite the use of a dCFA, a nondeterministic choice must be made (i.e., backtracking must be employed). It also checks and makes use of other properties, such as the so-called free-edge-choice property, and the existence of uniquely determined start nodes. As mentioned before, the precise discussion of these analysis techniques will be presented in a follow-up paper.

The CYK-style parsers for unrestricted HR grammars (plus edge-embedding 
rules) implemented in DiaGen [30] work for practical input with hundreds of nodes and edges, although their worst-case complexity is exponential. A closer comparison with PTD and PSR parsers shows its limits with larger input [24, Sect. 6].

\section{Future Work}

So far, PSR parsing has only been tested on small HR grammars; most of them are not relevant for practical modeling of graph and diagram languages (see Table 1). Indeed, HR grammars cannot generate graph languages like Petri nets or UML diagrams - not even the language of all graphs over some alphabet! For instance, HR languages do always have bounded treewidth. Therefore the authors have proposed a modest extension of this formalism $[10,11]$ : contextual $H R$ grammars allow to connect the right-hand side of a rule to a node that does not occur in the left-hand side nonterminal, but exists elsewhere in the context. In this way, some of the structural restrictions of HR languages can be overcome. Fortunately, PSR parsing for HR grammars can be extended to contextual HR grammars in a straightforward way: when shifting an edge attached to a context node, the parser must just be ready to match this node to a rule that has already been read. (This has already been implemented for the PTD parsing of graphs [12].)

However, the grammar in Example 2.6 indicates that HR grammars do have practical applications: for abstract meaning representations of natural language, HR grammars are being used in the natural language community [4]. Unfortunately the grammars occurring in this domain are not only huge, with thousands of rules, but also ambiguous, so that they do not lend themselves to PSR parsing. We are currently working on a generalized PSR parsing algorithm that pursues several PSR parses in parallel if states do have conflicts or if the free edge choice property is violated [23].

We will also study the relationship between PTD and PSR parsing. Bottomup string parsing is known to be more powerful than top-down string parsing in the sense that all top-down parsable languages are also bottom-up parsable, but not vice versa. PTD and PSR parsing are extensions of these string parsing approaches. Therefore, it appears to be an obvious assumption that PSR is more powerful than PTD. So far, we have no conclusive answers to this question. One of the challenges is to find an HR (or a contextual HR) language that has a PSR parser, but no PTD parser. The corresponding example for $\operatorname{LL}(k)$ and $\operatorname{LR}(k)$ string languages exploits that strings are always parsed from left to right - but this is not the case for PTD and PSR parsers.

\section{References}

\section{References}

[1] IJ.J. Aalbersberg, Andrzej Ehrenfeucht, and Grzegorz Rozenberg. On the membership problem for regular DNLC grammars. Discrete Applied Mathematics, 13:79-85, 1986. 
[2] Alfred V. Aho and Jeffrey D. Ullman. The Theory of Parsing, Translation, and Compiling, volume I: Parsing. Prentice-Hall, Inc., Upper Saddle River, NJ, USA, 1972.

[3] Laura Banarescu, Claire Bonial, Shu Cai, Madalina Georgescu, Kira Griffitt, Ulf Hermjakob, Kevin Knight, Philipp Koehn, Martha Palmer, and Nathan Schneider. Abstract meaning representation for sembanking. In Proc. 7th Linguistic Annotation Workshop at ACL 2013 Workshop, pages 178-186, 2013.

[4] David Chiang, Jacob Andreas, Daniel Bauer, Karl Moritz Hermann, Bevan Jones, and Kevin Knight. Parsing graphs with hyperedge replacement grammars. In Proc. 51st Ann. Meeting of the Assoc. for Computational Linguistic (Vol. 1: Long Papers), pages 924-932, 2013.

[5] G. Costagliola, A. De Lucia, S. Orefice, and G. Tortora. A parsing methodology for the implementation of visual systems. IEEE Transactions on Software Engineering, 23:777-799, 1997.

[6] Bruno Courcelle. The monadic second-order logic of graphs V: on closing the gap between definability and recognizability. Theoretical Computer Science, 80:153-202, 1991.

[7] Pierluigi Della Vigna and Carlo Ghezzi. Context-free graph grammars. Information and Control, 37(2):207-233, 1978.

[8] Franklin L. DeRemer. Simple LR(k) grammars. Comm. ACM, 14(7):453460, 1971.

[9] Frank Drewes. Recognising $k$-connected hypergraphs in cubic time. Theoretical Computer Science, 109:83-122, 1993.

[10] Frank Drewes and Berthold Hoffmann. Contextual hyperedge replacement. Acta Informatica, 52:497-524, 2015.

[11] Frank Drewes, Berthold Hoffmann, and Mark Minas. Contextual hyperedge replacement. In Proc. Applications of Graph Transformation with Industrial Relevance (AGTIVE'11), volume 7233 of LNCS, pages 182-197, 2012.

[12] Frank Drewes, Berthold Hoffmann, and Mark Minas. Predictive top-down parsing for hyperedge replacement grammars. In Proc. 8th Intl. Conf. on Graph Transformation (ICGT 2015), volume 9151 of LNCS, pages 19-34, 2015 .

[13] Frank Drewes, Berthold Hoffmann, and Mark Minas. Approximating Parikh images for generating deterministic graph parsers. In Software Technologies: Applications and Foundations - STAF 2016 Collocated Workshops, Revised Selected Papers, volume 9946 of LNCS, pages 112-128, 2016. 
[14] Frank Drewes, Berthold Hoffmann, and Mark Minas. Predictive shiftreduce parsing for hyperedge replacement grammars. In Proc. 8th Intl. Conf. on Graph Transformation (ICGT 2017), volume 10373 of LNCS, pages 106-122, 2017.

[15] Frank Drewes and Anna Jonsson. Contextual hyperedge replacement grammars for abstract meaning representations. In 13th Intl. Workshop on TreeAdjoining Grammar and Related Formalisms (TAG+13), pages 102-111, 2017.

[16] Joost Engelfriet and Grzegorz Rozenberg. Node replacement graph grammars. In Grzegorz Rozenberg, editor, Handbook of Graph Grammars and Computing by Graph Transformation. Vol. I: Foundations, chapter 1, pages 1-94. World Scientific, Singapore, 1997.

[17] Mariusz Flasiński. Characteristics of edNLC-graph grammar for syntactic pattern recognition. Computer Vision, Graphics, and Image Processing, 47(1):1-21, 1989.

[18] Mariusz Flasiński. Power properties of NLC graph grammars with a polynomial membership problem. Theoretical Computer Science, 201(1-2):189231, 1998.

[19] Mariusz Flasiński and Zofia Flasińska. Characteristics of bottom-up parsable edNLC graph languages for syntactic pattern recognition. In Leszek J. Chmielewski, Ryszard Kozera, Bok-Suk Shin, and Konrad W. Wojciechowski, editors, Computer Vision and Graphics - International Conference, ICCVG 2014, Proceedings, volume 8671 of LNCS, pages 195202. Springer, 2014.

[20] Reinhold Franck. A class of linearly parsable graph grammars. Acta Informatica, 10(2):175-201, 1978.

[21] S. Gilroy, A. Lopez, and S. Maneth. Parsing graphs with regular graph grammars. In Proc. 6th Joint Conf. on Lexical and Computational Semantics ( ${ }^{*} S E M$ 2017), pages 199-208, 2017.

[22] Annegret Habel. Hyperedge Replacement: Grammars and Languages, volume 643 of LNCS. Springer, 1992.

[23] Berthold Hoffmann and Mark Minas. Generalized predictive shift-reduce parsing for hyperedge replacement graph grammars. Appears in Proc. Language and Automata Theory and Applications - 13th International Conference, LATA 2019, LNCS.

[24] Berthold Hoffmann and Mark Minas. Generating efficient predictive shiftreduce parsers for hyperedge replacement grammars. In Martina Seidl and Steffen Zschaler, editors, Software Technologies: Applications and Foundations - STAF 2017 Collocated Workshops, Revised Selected Papers, volume 10748 of $L N C S$, pages 76-91. Springer, 2017. 
[25] Manfred Kaul. Practical applications of precedence graph grammars. In Hartmut Ehrig, Manfred Nagl, Grzegorz Rozenberg, and Azriel Rosenfeld, editors, Graph-Grammars and Their Application to Computer Science, volume 291 of $L N C S$, pages 326-342, 1986.

[26] Donald E. Knuth. On the translation of languages from left to right. Information and Control, 8(6):607 - 639, 1965.

[27] Clemens Lautemann. The complexity of graph languages generated by hyperedge replacement. Acta Informatica, 27:399-421, 1990.

[28] Philip M. Lewis II and Richard Edwin Stearns. Syntax-directed transduction. J. ACM, 15(3):465-488, 1968.

[29] Helmut J. Ludwigs. A LR-like analyzer algorithm for graphs. In Reinhard Wilhelm, editor, GI - 10. Jahrestagung, Saarbrücken, 30. September - 2. Oktober 1980, Proceedings, volume 33 of Informatik-Fachberichte, pages 321-335, 1980.

[30] Mark Minas. Diagram editing with hypergraph parser support. In Proc. 1997 IEEE Symposium on Visual Languages (VL'97), Capri, Italy, pages 226-233, 1997.

[31] Rasmus Pagh and Flemming Friche Rodler. Cuckoo hashing. In Friedhelm Meyer auf der Heide, editor, Algorithms - ESA 2001, volume 2161 of $L N C S$, pages 121-133, 2001.

[32] Theodosios Pavlidis. Linear and context-free graph grammars. J. ACM, 19(1):11-22, 1972.

[33] Terrence W. Pratt. Pair grammars, graph languages and string-to-graph translations. Journal of Computer and System Sciences, 5:560-595, 1971.

[34] Walter Vogler. Recognizing edge replacement graph languages in cubic time. In H. Ehrig, H.-J. Kreowski, and G. Rozenberg, editors, Proc. Fourth Intl. Workshop on Graph Grammars and Their Application to Comp. Sci., volume 532 of $L N C S$, pages 676-687. Springer, 1991. 